\title{
Holomorphic Extension Problem for Standard Real Submanifolds of Second Kind
}

\author{
By \\ Isao NARUKI
}

\section{Contents}

Introduction

§1. Standard Real Submanifolds

1. 1. Definition of standard real submanifolds

1.2. Elementary study of standard real submanifolds of second kind

§2. Holomorphic Hulls for Open Subsets of $D_{0}$

2.1. Basic continuation theorem

2. 2. Application to $D_{0}$

§3. Approximation Theorems

3. 1. Approximation theorem on $U(n)$

3.2. Application to some type of vector fields

$\S 4$. Problem $H$ for $D_{0}$

4.1. A local parametrization of $D_{0}$

4. 2. Solution of Problem $H$ for $D_{0}$

§5. Application to Standard Real Submanifold of Second Kinc

5.1. Basic notation and definitions

5. 2. $\forall$-indefinite case

5. 3. Dually $\forall$-indefinite case

Received January 16, 1970. 


\section{Introduction}

Recently real submanifolds of complex manifolds have been studied by several complex analysts, differential geometers. Ever since Lewy [5] studied the holomorphic extension problem for pseudo-convex hypersurfaces in $C^{2}$, Lewy [6], Wells [12], [13], Greenfield [2] obtained many results on the hull of holomorphy of lower-dimensional real submanifolds in complex manifolds. On the other hand Tanaka [9], [10], [11] studied the pseudo-conformal equivalence between real submanifolds as an example of geometry of differential systems and at the same time he introduced in a group-theoretic manner an ideal class of real submanifolds in complex vector spaces which are called 'standard real submanifolds'. In this paper we attempt to study the hulls of holomorphy for a special kind of standard real submanifolds, i.e., those of the second kind and further to solve the holomorphic extension problem for those real submanifolds. We shall now give a precise formulation of the holomorphic extension problem.

Let $M$ be a real submanifold of a complex manifold $\widetilde{M}$ and $T^{c}(M)$, $T^{c}(\widetilde{M})$ the complexifications of the tangent bundles $T(M), T(\widetilde{M})$ of $M, \widetilde{M}$ respectively. For any $p \in M, T_{p}^{c}(M)$ is a subspace of $T_{p}^{c}(\widetilde{M})$ and we shall denote by $\mathcal{S}_{p}$ the intersection of $T_{p}^{C}(M)$ with $T_{p}^{(0,1)}(\widetilde{M})$, that is,

$$
\mathcal{S}_{p}=T_{p}^{C}(M) \cap T_{p}^{(0,1)}(M) .
$$

Here we have set $T_{p}^{(0,1)}(\widetilde{M})=\left\{x+i I x ; x \in T_{p}(\widetilde{M})\right\}$. ( $I$ is the automorphism of $T_{p}(\widetilde{M})$ defining the complex structure of $T_{p}(\widetilde{M})$.) We assume that $\operatorname{dim} \mathcal{S}_{p}$ does not depend on $p$. Then we obtain a unique vector bundle $\mathcal{S}$ whose fiber at each $p$ of $M$ is $\mathcal{S}_{p}$. This subbundle $\mathcal{S}$ of $T^{c}(M)$ is called the tangential Cauchy-Riemann bundle of $M$. A continuous function $f$ on an open subset $U$ of $M$ is called $\mathcal{S}$-holomorphic in $U$ if it is a (distribution) solution of the following differential equation

$$
{ }^{\forall} X \in \Gamma_{v}(\mathcal{S}): X f=0 .
$$

This equation is called the tangential Cauchy-Riemann equation of $M$. From definition of $\mathcal{S}$ it follows immediately that the restriction of a 
holomorphic function to the intersection of its domain with $M$ is always $\mathcal{S}$-holomorphic. The converse is, however, not true in general. Thus it is an interesting problem to examine under various condition on $M$ to what extent an $S$-holomorphic function can be continued into a holomorphic function. More precisely it may be formulated as the following

Problem H. Let $M, \widetilde{M}$ be as above. Given an open subset $U$ of $M$, find an open subset $\widetilde{U}$ of $\widetilde{M}$, whose closure contains $U$ with the property that one can extend every $\mathcal{S}$-holomorphic function in $U$ to a continuous function $v$ in $U \cup \widetilde{U}$ so that $v$ is holomorphic in $\widetilde{U}$.

This is the holomorphic extension problem. The investigation of this may be divided into the following two steps. The first is to show that a holomorphic function $u$ defined near $U$ can be holomorphically continued to a neighbourhood of $U \cup \widetilde{U}$ and that this $\widetilde{U}$ can be chosen at once for all $u$. The next is to show that in the set of $\mathcal{S}$-holomorphic functions on $U$ there densely exist restrictions to $U$ of holomorphic functions near $U$. The first step may be done by the method of Wells and Greenfield, but we do not utilize this since a much simpler approach is possible when $M$ is a standard real submanifold. (However it should be noted that our procedure is essentially based on the same idea as theirs, i.e., the analytic discs.) As opposed to the generality of the results obtained in [13], [2], we were able to make a fitting choice of $U$ because the situation is simple in our present case. In fact, for a standard real submanifold $M$ of second kind in a complex vector space $N$ we can canonically assign a domain $D$ of $N$ whose closure contains $M$ in such a way that, when $M$ is the Šilov boundary of a non degenerate Siegel domain of the second kind, the corresponding domain is nothing but the Siegel domain itself, moreover, in this case, the above $\widetilde{U}$ can be taken so that $U \cup \widetilde{U}$ is an open subset of $M \cup D$. As for the second step our discussion here is goup-theoretic and is based on an approximation theorem which is an analogue of the famous theorem of Harish-Chandra, Nelson and Gårding in the representation 
theory, but our theorem is very special and much more complicated. In the near future the method we exploit will be developed into a theory of differential equations admitting a Lie transformation group and is also interesting in its own right.

Among standard real submanifolds of second kind two classes of them are of particular importance, one is the class of so called 'totally indefinite' ones and the other is the class of Šilov boundaries of non degenerate Siegel domains of the second kind. One of the most remarkable facts concerning totally indefinite standard real submanifolds is that every $\mathcal{S}$-holomorphic function on such a submanifold is the restriction of an entire holomorphic function in the ambient complex vector space, moreover it is also locally true, that is, every $\mathcal{S}$-holomorphic functon on an open subset of the real submanifold can be extended into a unique holomorphic function in a fixed open subset of the ambient space containing the domain of the original function. As a result we obtained in this case a hypoellipticity theorem in the real analytic category on the tangential Cauchy-Riemann equation which is, however, not elliptic. Further it is also proved that the space of $\mathcal{S}$-holomorphic functions on an open subset of a totally indefinite standard real submanifold is a Montel space, so that the finiteness theorem is obtained for $\mathcal{S}$-holomorphic vector bundles over compact locally standard real submanifolds. However, this theorem holds for a much wider class of real submanifolds [7]. The method of [7] is based on an estimate of Hörmander used in connection with the theory of hypoelliptic second order differential equations [3]. But the procedure used here leads us to a conjecture on Problem $H$ for arbitrary totally indefinite real submanifolds. For two integers $n, k$ such that $n \geq 2$, $0 \leq k \leq n^{2}-1$, it is possible to construct a totally indefinite standard real submanifold of real dimension $2 n+k$ in $\boldsymbol{C}^{n+k}$, thus its real codimension being $k$.

Šilov boundaries of non-degenerate Siegel domains of the second kind are all contained in some wider class of standard real submanifolds, which is dual, in certain sense, for the class of totally indefinite ones. 
For this dual class we also have solved Problem $H$ in slight stronger form. However, the meaning of the resilt is not so clear in this case as in totally indefinite case. As examples of compact locally standard real submanifold of this type, there are the Šilov boundaries of the classical domains $I_{p, q}(p \neq q)$, which are all well-known [4], [8].

Because of the restriction of the argument to standard real submanifolds we have received an important simplification of the method, i.e. the homomorphisms between standard real submanifolds. (This was informed by Tanaka in connection with the problem on the existence of a bounded global $\mathcal{S}$-holomorphic function on staindard real submanifolds.) In fact this eliminates the necessity to attack each standard real submanifold, at least as far as concerning our problem, and instead we may only study a special one denoted by $D_{0}$ (or $M^{0}$ ). This is the Šilov boundary of certain Siegel domain equivalent to a classical domain and for this reason the treatment is very handy.

Now we shall shortly describe the construction of this paper. In $\$ 1$ we study general real submanifolds in complex manifolds and define standard real submanifolds. We study also elementary properties of standard real submanifolds of the second kind, for example, a simple method of ti:eir construction, komorphism, and so 0 . . Most of the materials of this section are due to [10], [11]. In $\$ 2$ we investigate the shape of the holomorphic hull of an open subset of $D_{0}$. In $\S 3$ we study some special kind of differential operators on a trivial $U(n)$ bundle which are invariant under the right (or left) operation of $U(n)$ in order to obtain an approximation theorem for solutions of those differential operators. The results can be directly extended also for right invariant differential operators on an arbitrary $U(n)$-bundle on a $C^{\infty}$ manifold. However, we do not include this since it is trivial. In $\S 4$ we give a cosvenient parametrization of $D_{0}$ in order to apply the result of $\S 3$ to $D_{0}$. Further combining the result of $\S 2$ we solve Problem $H$ for $D_{0}$ in somewhat stronger version. In $\S 5$ we extend the result of $\$ 4$ for almost all standard real submanifolds of the second kind, i.e. the stable ones. First we shall concern ourselves with totally 
indefinite ones and mention the conjecture indicated already. Next we study the dual class of standard real submanifolds, and as a result we solve Problem $H$ for Šilov boundaries of non-degenerate Siegel domains of the second kind.

Here we shall give some notational conventions frequently used. For two subsets $A, B$ of a vector space $W$ we denote the set $\{a+b$; $a \in A, b \in B\}$ by $A+B$. For two vector spaces $V$ and $W$ we shall regard, in canonical way, $V$ and $W$ as subspaces of the direct sum $V+W$. If we wish to distinguish between elements of $V$ and those of $W, V+W$ will be considered to be also the direct product $V \times W$, and then $(x, y)$ means $x+y$ when $x, y$ lie in $V, W$ respectively. The letter $I$ will mostly be used to denote the linear automorphism of a complex vector space sending each element to that multiplied by $i=\sqrt{-1}$. If $A$ is an endomorphism of a (real or complex) vector space $V, D_{V}(A)$ denotes the determinant of $A$. When $A$ maps a subspace $W$ of $V$ into itself, then $D_{W}(A)$ means the determinant of $\left.A\right|_{W}$. We also denote the determinant of a matrix $A$ by $D(A)$. For a vector space $V, I_{V}$ is the identity map of $V$ winile $I_{n}$ is the identity matrix of type $(n, n)$. In $\$ \S 3 \sim 4$ we use capital roman letter to denote matrices and capital gothic letters to denote matrix groups. In other sections Lie groups are usually denoted by capital roman letters. For two subsets $A, B$ of a topological space $A$, to say that $A$ is an open (resp. closed) subset of $B$ means that $A$ is open (resp. closed) in the relative topology of $B$ induced from $A$. Unless smoothness is stated explicitly, we assume the differentiability of class $C^{\infty}$. For a (real or complex) vector bundle $E$ over a manifold $M$ we shall denote by $\underline{E}$,

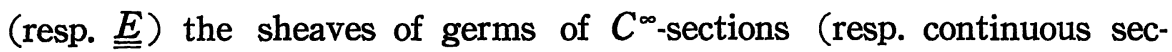
tions). For $p \in M, E_{p}$ denotes the fiber of $E$ over $p$. Similarly $S_{p}$ is a stalk of $S$ over $p$ if $S$ is a sheaf over $M . \quad \Gamma_{\Omega}(S)$ is the set of sections over $\Omega$ and $\Gamma(S)$ denotes the set of global sections of $S$. For simplicity we use $\Gamma_{\Omega}(E), \Gamma(E)$ instead of $\Gamma_{\Omega}(\underline{E}), \Gamma(\underline{E})$. For a complex manifold $X, H(X)$ denotes the set of holomorphic functions on $X$. For a relatively compact subset $S$ of $X$ we shall denote by $\widehat{S}_{X}$ the 
subset $\left\{x \in X:|f(x)| \leq \sup _{z \in S}|f(z)| f \in H(X)\right\}$ of $X$. Here the author apologizes to the reader for the disharmony of notations which arises from the comparative independence of each section. In fact it might be said that this paper grew out of many small papers.

In this occasion the author thanks to Prof. Tanaka for suggesting the idea of homomorphism and a number of stimulating conversations. $\mathrm{He}$ also thanks to Prof. Matsuura for his constant encouragement and critical advices.

\section{§1. Standard Real Submanifolds}

1.1. Definition of Standard Real Submanifolds. To motivate our discussion we shall begin with the study of general real submanifolds in complex manifolds.

Let $M, \widetilde{M}, \mathcal{S}$ be as in the introduction and define a distribution (in the sense of Chevalley) $D$ on $M$ setting

$$
D_{p}=\left\{\operatorname{Re} x: x \in \mathcal{S}_{p}\right\}
$$

where $\operatorname{Re} x$ denotes the real part of $x$. Assume that there exists a series of distributions $D=D^{1} \subseteq D^{2} \subset, \cdots, D^{\mu}=T(M)$ such that

$$
\underline{D}^{i}=\left[\underline{D}^{i-1}, \underline{D}\right]+\underline{D}^{i-1} \quad(i \geq 2)
$$

where $\underline{D}^{k}$ denotes the sheaf of germs of smooth sections $D^{k}$. Then

$$
\left[\underline{D}^{j} ; \underline{D}^{k}\right] \subseteq \underline{D}^{j+k}
$$

Let $\mathrm{g}^{i}$ denote the quotient bundle of $D^{i}$ by $D^{i-1}(i \geq 2)$ and set $\mathrm{g}^{1}=D^{1}$, $\mathfrak{m}=\mathfrak{g}^{1} \oplus \mathfrak{g}^{2} \oplus \cdots \oplus \mathfrak{g}^{\mu}$. Then the bracket operation induces a Lie algebra structure of $\Gamma(\mathfrak{m})$, such that

$$
\left[\underline{\mathfrak{g}^{j}}, \underline{\mathrm{g}}^{k}\right] \subseteq \underline{\mathrm{g}}^{j+k}
$$

where we have put $\mathrm{g}^{k}=0$ if $k>\mu$.

One can easily show that

$$
[f X, g Y]=f g[X, Y] \quad f, g \in C^{\infty}(M) \quad X, Y \in \Gamma(\mathfrak{m}) .
$$

Thus this Lie algebra structure is defined pointwisely, i.e., $\mathfrak{n t}_{p}=\mathrm{g}_{p}^{1}+\cdots$ $+\mathrm{g}_{p}^{\mu}(p \in M)$ has also the canonical Lie algebra structure such that 


$$
\left[X_{p}, Y_{p}\right]=[X, Y]_{p} \quad X, Y \in \Gamma(\mathfrak{m}) .
$$

The properties of $\mathfrak{m}_{p}$ are the followings

(i) $\mathfrak{m}_{p}$ is generated by $\mathfrak{g}_{p}^{1}$

(ii) $\left[\mathrm{g}_{p}^{j}, \mathrm{~g}_{p}^{k}\right] \subseteq \mathrm{g}_{p}^{j+k}$

(iii) $[\operatorname{Re} x, \operatorname{Re} y]=[\operatorname{Re}(i x), \operatorname{Re}(i y)] \quad x, y \in S_{p}$.

Let $I$ be the linear isomorphism $\operatorname{Re} x \rightarrow \operatorname{Re}-i x$ of $D_{p}$ onto itself, then (iii) is equivalent to

$$
\text { (iii) }^{\prime}[x, y]=[I x, I y] \quad x, y \in D_{p} .
$$

For $p \in M \mathfrak{m}_{p}$ is called the Levi-Tanaka algebra of $M$ at $p$. We assume that for $(p, q) \in M \times M$ there exists a Lie algebra isomorphism $\sigma$ of $\mathfrak{m}_{p}$ onto $\mathfrak{m}_{q}$ such that $\sigma\left(\mathfrak{g}_{p}^{i}\right)=\mathfrak{g}_{q}^{i}$ and

$$
\sigma(I x)=I \sigma(x) \quad x \in g_{p}^{1} .
$$

A real submanifold satisfying all hypotheses assumed so far is called a strongly regular submanifold.

From now on we shall discuss only strongly regular submanifolds. Now a question arises: Suppose that $\mathfrak{m}=g_{1}+\cdots+g_{\mu}$ where $g_{1}$ is a complex vector space, is a finite-dimersicial Lie algebra satisfying following properties

(i) $\mathfrak{m}$ is generated by $\mathfrak{g}_{1}$

(ii) $\quad\left[\mathfrak{g}_{j}, \mathfrak{g}_{k}\right]=\mathfrak{g}_{j+k} \quad\left(\mathfrak{g}_{l}=0\right.$ if $\left.l>\mu\right)$

(iii) $[I x, I y]=[x, y] \quad x, y \in g_{1}$

where $I$ is the linear isomorphism of $g_{1}$ defining the complex structure of $\mathrm{g}_{1}$. Is there a strongly regular real submanifold $M$ in certain complex manifold such that the Levi-Tanaka algebra at each point $p$ of $M$ is isomorphic to $\mathfrak{m}$ ? This is affirmatively answered in the following way.

Let $\mathfrak{m}^{c}$ be the complexification $\mathfrak{m}+i \mathfrak{m}$ of $\mathfrak{m}, M^{c}$ the simply connected Lie group whose Lie algebra is $\mathfrak{m}^{c}$, and exp the exponential map of $\mathfrak{m}^{c}$ into $M^{c}$. Then exp is a holomorphic isomorphism of $\mathfrak{m}^{c}$ onto $M^{c}$ since $\mathfrak{i n}^{c}$ is nilpotent. Set

$$
S=\left\{x+i I x ; x \in \mathrm{g}_{1}\right\}, \quad N=\bar{S}+\mathrm{g}_{2}^{C}+\cdots+\mathrm{g}_{\mu}^{C} .
$$


$S, \bar{S}$ are abelian subalgebra of $\mathfrak{m}^{c}$ and $N$ is an ideal of $\mathfrak{m}^{c}$. Set further $M^{\prime}=\exp (\mathfrak{m}), H=\exp (S), N=\exp (N) . \quad M^{\prime}, H$ are Lie subgroups of $M^{c} . N$ is a normal subgroup of $M^{c}$. Since exp is one to one and $\mathfrak{m} \cap S=(0)$, we obtain

$$
M^{\prime} \cap H=(e) .
$$

In other words the restriction to $M^{\prime}$ of the canonical projection $\pi: M^{c}$ $\rightarrow M^{c} / H$ is one to one on $M^{\prime}$. Then real submanifold $M=\pi\left(M^{\prime}\right)$ of complex manifold $M^{c} / H$ is the desired real submanifold. To see this let $\sigma_{p}$ be the isomorphism of $\mathfrak{m}^{c}$ onto $T_{p}\left(M^{c}\right)$ defined by the MaurerCartan form of $M^{c}$. Then the sequence

$$
0 \rightarrow S \stackrel{l}{\rightarrow} \mathfrak{m}^{c} \stackrel{d \pi_{p} \circ \sigma_{p}}{\longrightarrow} T_{\pi(p)}\left(M^{c} / H\right) \rightarrow 0
$$

is exact where $l$ is the inclusion map. Denote by $f_{p}$ the isomorphism of $\mathfrak{m}^{c} / S$ onto $T_{\pi(p)}\left(M^{c} / H\right)$ such that the following diagram is commutative:

$$
\begin{aligned}
& { }_{\mathfrak{m l}} \stackrel{d \pi_{p} \circ \sigma_{p}}{\longrightarrow} T_{\pi(p)}\left(M^{c} / H\right) \\
& \text { canonical } \downarrow \quad\left\lceil f_{p}\right. \\
& \mathfrak{m}^{c} / S
\end{aligned}
$$

Let $\mathfrak{p}$ denote the restriction to $\mathfrak{m}$ of the canonical projection $\mathfrak{m}^{c} \rightarrow \mathfrak{m}^{c} / S$. $\mathfrak{p}$ is an isomorphism into $\mathfrak{m}^{c} / S$ since $\mathfrak{m} \cap S=(0)$. For $p \in M^{\prime}$ we have

$$
\mathfrak{p}(\mathfrak{i n})=f_{p}^{-1}\left(T_{\pi(p)}(M),\right.
$$

while we obtain by simple calculation

$$
\mathfrak{p}(\mathfrak{m})^{c} \cap\left(\mathfrak{m}^{c} / S\right)^{(0,1)}=\mathfrak{p}^{c}(S)
$$

where $\mathfrak{p}^{c}=\mathfrak{p} \otimes C: \mathfrak{m}^{c} \rightarrow\left(\mathfrak{m}^{c} / S\right)^{c}$. (For a complex vector space $V$ we shall denote by $V^{(0,1)}$ the subspace of $V^{c}$ given by

$$
V^{(0,1)}=\{x+i I x: x \in V\}
$$

where $I$ is the automorphism of $V$ which defines the complex structure of $V$.)

Therefore we obtain

$$
\sigma_{p}(S)=\left(d \bar{\pi}_{p}\right)^{-1}\left(\mathcal{S}_{\pi(p)}\right)
$$


where $\mathcal{S}$ is the tangential Cauchy-Riemann bundle of $M$ and $\bar{\pi}$ is the restriction to $M^{\prime}$ of $\pi$. This implies that

$$
\left(d \bar{\pi}_{p}\right)^{-1}\left(D_{p}^{i}\right)=\left\{\sigma_{p}(x): x \in \mathfrak{g}_{1}+\cdots+\mathrm{g}_{i}\right\}
$$

since $\mathfrak{m}$ can be regarded as the Lie algebra of left invariant vector fields $M^{\prime}$. Therefore $\mathfrak{m}_{p}=\sum_{i=1}^{\mu} D_{p}^{i} / D_{p}^{i-1}$ can be canonically identified with $\mathfrak{m}=\sum_{i=1}^{\mu} g_{i}$ and this identification is a Lie algebra isomorphism.

A finite-dimensional Lie algebra in with properties (i), (ii), (iii) is called a fundamental algebra of the $\mu$-th kind and the real submanifold $M$ constructed above is called the standard real submanifold corresponding to $\mathfrak{m}$, which will be denoted by $M(\mathfrak{m})$ hereafter.

Remark. Since $\boldsymbol{N} \cap H=(e)$ the restriction to $\boldsymbol{N}$ of the canonical projection $M^{c} \rightarrow M^{c} / H$ is a holomorphic isomorphism onto $M^{c} / H$, the inverse of which we shall denote by $T^{\prime}$. Since $\exp \mid N$ is a holomorphic isomorphism of $N$ onto $N, T=(\exp )^{-1} \circ T^{\prime}$ is also a holomorphic isomorphism of $M^{c} / H$ onto $N$. Thus, if we identify $M^{c} / H$ with $N$ by $T, M$ can be considered as a real submanifold of $N$, further extending the linear isomorphism of $\mathrm{g}_{1}$ onto $\bar{S}$ given by $x \rightarrow \frac{1}{2}(x-i I x)$ to a linear isomorphism of $\mathrm{g}_{1}+\mathrm{g}_{2}^{C}+\cdots+\mathrm{g}_{\mu}^{C}$ onto $N$ in obvious manner, we shall consider $M$ as a real submanifold of $\mathrm{g}_{1}+\mathrm{g}_{2}^{C}+\cdots+\mathrm{g}_{\mu}^{C}$.

Remark. By $\rho(p)$ we shall denote the canonical left operation on $M^{c} / H$ of an element $p \in M^{c}$. Then for $p \in M^{\prime} \rho(p)$ leaves $M$ invariant. Thus the holomorphic transformation group $\rho\left(M^{\prime}\right)$ operates transitively on $M$. In other words $M$ is homogeneous.

Above arguments show that standard real submanifolds are the most typical and important ones.

1.2. Elementary Study of Standard Real Submanifolds of the Second KIND. From now on we shall concern ourselves only with standard real submanifolds corresponding to fundamental Lie algebra of the second kind, which will be called shortly '2-standard real submanifolds.' First we give a method of construction of all 2-standard real submanifolds. 
Let $V$ be an $n$-dimensional complex vector space and $I$ the linear isomorphism of $V$ onto itself which defines the complex structure of $V$, i.e., $I$ maps $x$ into $\sqrt{-1} x$ for $x \in V$, let $H(V)$ denote the vector space of hermitian forms on $V^{*}$, i.e.,

$$
H(V)=\left\{\alpha \in S_{R}^{2}(V) ; I \otimes I(\alpha)=\alpha\right\}
$$

where $S_{R}^{2}(V)$ denotes the vector space of symmetric elements of $V \otimes_{R} V$. Further let $\pi$ be a linear mapping of $H(V)$ onto a vector space $W$ and set $\mathfrak{g}_{1}=V, \mathrm{~g}_{2}=W, \mathfrak{m}(\pi)=\mathrm{g}_{1}+\mathrm{g}_{2}$. Then we can define the bracket operation $[$,$] on \mathfrak{m}(\pi) \times \mathfrak{m}(\pi)$ so that $\mathfrak{m}(\pi)$ is a fundamental Lie algebra of second kind. For this we must set

$$
\left[g_{1}, g_{2}\right]=\left[g_{2}, g_{2}\right]=(0) \text {. }
$$

As to $[$,$] on g_{1} \times g_{1}$ we set

$$
[x, y]=\frac{1}{4} \pi(x \otimes I y-y \otimes I x-I x \otimes y+I y \otimes x) \quad x, y \in \mathrm{g}_{1} .
$$

It is easily checked that $\mathfrak{m}(\pi)$ satisfies properties (i), (ii), (iii). We shall denote by $M(\pi)$ the standard real submanifold of $\mathrm{g}_{1}+\mathrm{g}_{2}^{C}$ corresponding to $\mathfrak{m}(\pi)$. By direct computation we have

$$
M(\pi)=\left\{\left(x, y-\frac{1}{4} i[x, I x]\right) \in \mathrm{g}_{1}+\mathrm{g}_{2}^{C}: x \in \mathrm{g}_{1}, y \in \mathrm{g}_{2}\right\} .
$$

Here we write $(x, z)$ for $x+z$, regarding $g_{1}+g_{2}^{C}$ as $g_{1} \times g_{2}^{C}$.

We can obtain any 2-standard submanifold in this way. To see this let $\mathfrak{m}=\mathfrak{g}_{1}+\mathfrak{g}_{2}$ be a fundamental Lie algebra of the second kind and set $V=\mathrm{g}_{1}, W=\mathrm{g}_{2}$. We define a linear mapping $\phi$ of $W^{*}$ into $H\left(V^{*}\right)\left(=H(V)^{*}\right)$ setting

$$
\phi(\alpha)(x, y)=\alpha([x, I y]) .
$$

Then $\phi$ is one to one because of property (i) of $\mathfrak{m}$. Hence $\pi={ }^{t} \phi$ is a linear mapping of $H(V)$ onto $W$, and we have $\mathfrak{m}=\mathfrak{m}(\pi)$. Details of the verification shall be left to the reader.

Now we shall introduce 'homomorphism' between standard real submanifolds, which makes it unnecessary to study all standard real submanifolds. (In fact it suffices to study only special one, namely 
$M^{0}=M\left(i d_{H(V)}\right)$.

Consider the following commutative diagram

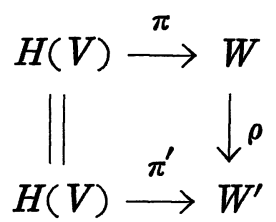

where $\pi, \pi^{\prime}, \rho$ are all surjections. Then the linear mapping $\tilde{\rho}=i d_{V} \oplus^{c}$ of $N=V \oplus W^{c}$ onto $N^{\prime}=V \oplus W^{\prime c}$ maps $M(\pi)$ onto $M\left(\pi^{\prime}\right), \tilde{\rho}$ is obviously a holomorphic mapping and the restriction $\hat{\rho}$ to $M(\pi)$ of $\tilde{\rho}$ is called homomorphism induced by $\rho$. Now consider the following diagram:

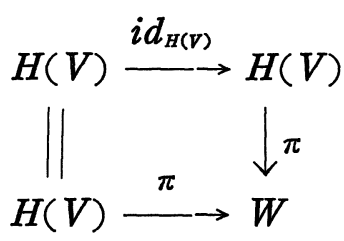

Then we obtain homomorphism $\hat{\pi}$ of $M^{0}$ onto $M(\pi)$. Thus every 2-standard real submanifold is the homomorphic image of $M^{0}$. This fact allows us to deduce many information concerning our problem for $M(\pi)$ from those for $M^{0}$. For example, if $f$ is $\mathcal{S}$-holomorphic in an open set $U$ of $M(\pi)$, then $\hat{\pi}^{*}(f)$ is also $\mathcal{S}$-holomorphic on $\hat{\pi}^{-1}(U)$. This is easily seen from

$$
d \hat{\pi}_{p}\left(\mathcal{S}_{p}^{0}\right)=\mathcal{S} \hat{\bar{\pi}}_{(p)}
$$

where $\mathcal{S}^{0}, \mathcal{S}$ are the tangential Cauchy-Riemann bundles of $M^{0}, M(\pi)$ respectively. Conversely if, for a continuous function $f$ on an open subset $U$ of $M(\pi), \hat{\pi}^{*}(f)$ is $\mathcal{S}$-holomorphc in $\hat{\pi}^{-1}(U)$, then $f$ is also $\mathcal{S}$-holomorphic in $U$. Further, if $f$ is the restriction of a holomorphic function in a neighbourhood of $U, \hat{\pi}^{*}(f)$ is also the restriction of a holomorphic function in a neighbourhood of $\hat{\pi}^{-1}(U)$. But it is not quite evident that $f$ is the restriction of a holomorphic function defined near $U$ whenever $\hat{\pi}^{*}(f)$ is the restriction of a holomorphic function defined near $\hat{\pi}^{-1}(U)$. Fortunately it is true. This follows from

Proposition 1.2.1. Let $\mathcal{S}$ be the tangential Cauchy-Riemann 
bundle of a real submanifold $M$ of a complex manifold $M$ (assuming that $S$ is well-defined). Suppose that (2) complex fiber dimension of $\mathcal{S}+$ complex dimension of $\widetilde{M}=$ real dimension of $M$. Then every holomorphic function $u$ on a domain $\widetilde{U}$ of $\widetilde{M}$ such that $U \cap$ $\widetilde{M} \neq \phi$ and $\left.u\right|_{\tilde{v}_{\Pi M}}=0$ must vanish identically in $U$.

Proof. Condition (2) implies that for $p \in \widetilde{M} T_{p}(\widetilde{M})$ is the unique complex subspace of itself containing $T_{p}(M)$. To show this let $p_{1}$ be the projection of $T_{p}^{C}(\widetilde{M})$ onto $T_{p}^{(1,0)}(\widetilde{M})$ in the direct sum $T_{p}^{C}(\widetilde{M})$ $=T_{p}^{(1,0)}(\widetilde{M}) \oplus T_{p}^{(0,1)}(\widetilde{M})$ and $\check{p}_{1}$ the restriction to $T_{p}^{C}(M)$ of $p_{1}$. Then condition (2) implies that

dimension of $T_{p}^{C}(M)$-dimension of Kernel of $\check{p}_{1}$

=dimension of $T_{p}^{(0,1)}(\widetilde{M})$

since $\mathcal{S}_{p}=$ Kernel of $\check{p}_{1}$. Thus $\check{p}_{1}$ is onto; in other words $T_{p}^{C}(M)$ $+T_{p}^{(0,1)}(\widetilde{M})=T_{p}^{C}(\widetilde{M})$. Now let $L$ be a complex subspace of $T_{p}(\widetilde{M})$ containing $T_{p}(M)$, then we have

$$
\begin{aligned}
& T_{p}^{C}(M) \subseteq L^{c} \\
& L=L^{(0,1)} \bigoplus L^{(0,1)}
\end{aligned}
$$

where $L^{(0,1)}=L^{c} \cap T_{p}^{(0,1)}(\widetilde{M}), L^{(0,1)}=L^{c} \cap T_{p}^{(0,1)}(\widetilde{M})$. Therefore

$$
\begin{aligned}
T_{p}^{c}(\widetilde{M}) & =T_{p}^{c}(M)+T_{p}^{(0,1)}(\widetilde{M})=L^{(1.0)}+L^{(0,1)}+T_{p}^{(0,1)}(\widetilde{M}) \\
& =L^{(1,0)}+T_{p}^{(0,1)}(\widetilde{M})
\end{aligned}
$$

which implies that $L^{(1,0)}=T_{p}^{(1,0)}(\widetilde{M})$. Consequently we have

$$
L=T_{p}(\widetilde{M}) .
$$

Our assertion is proved.

Now let $u$ be a holomorphic function in a domain $\widetilde{U}$ of $\widetilde{M}$ such that $\widetilde{U} \cap M \neq \phi$ and $\left.u\right|_{\tilde{v} \cap M}=0$. Suppose that $u$ does not vanish identically. Then we obtain a series of proper analytic sets $V_{1}, V_{2}, \cdots$ of $\widetilde{U}$ such that $V_{1}=\{z \in \widetilde{U}: u(z)=0\}, V_{k}$ is the set of singular points of $V_{k-1}(k \geq 2)$. Since $\operatorname{dim} V_{k}<\operatorname{dim} V_{k-1}$ and since $V_{1} \supseteq M \cap \widetilde{U}$, it holds for some $i$ that

$$
V_{i} \supseteq M \cap \widetilde{U},\left(V_{i} \backslash V_{i+1}\right) \cap(M \cap \widetilde{U}) \neq \phi .
$$


(For $V_{k} \supseteq M \cap \widetilde{U},\left(V_{k} \backslash V_{k+1}\right) \cap(M \cap \widetilde{U})=\phi$ imply $V_{k+1} \supset M \cap \widetilde{U}$ ) Choose

Then

$$
p \in\left(V_{i} \backslash V_{i+1}\right) \cap(M \cap \widetilde{U}) .
$$

$$
T_{p}\left(V_{i}\right) \supseteq T_{p}(M) \text { since } V_{i} \supseteq(M \cap \widetilde{U}) .
$$

As we have showed above this implies $T_{p}\left(V_{i}\right)=T_{p}(\widetilde{M})$ i.e., $\operatorname{dim} V_{i}$ $=\operatorname{dim} \widetilde{M}$ which contradicts the properness of $V_{i}$. Thus $u=0$ is proved.

Q.E.D.

Let us now study the aspect of homomorphisms more in detail. Let $M^{\prime}\left(\mathrm{m}^{0}\right), M^{\prime}(\pi)$ be the simply connected Lie groups corresponding to $\mathfrak{m}^{0}\left(=\mathfrak{m}\left(i d_{H(V)}\right)\right), \mathfrak{m}(\pi)$ respectively. Then the linear map $i d_{V} \oplus \pi$ of $\mathfrak{m}^{0}$ onto $\mathfrak{m}(\pi)$ is a Lie algebra homomorphism. Let $\check{\pi}$ be the homomorphism of $M^{\prime}\left(\mathrm{m}_{0}\right)$ onto $M(\pi)$ induced by $i d_{v} \oplus \pi$, the Lie algebra of the kernel $K(\pi)$ of $\check{\pi}$ is the kernel of $\pi$ when one regards $H(V)$ as a subspace of $\mathfrak{m}^{0}=V+H(V)$. By direct inspection we obtain

$$
\begin{aligned}
0 \rightarrow K(\pi) \rightarrow M^{\prime}\left(\mathfrak{m}^{0}\right) & \stackrel{\check{\pi}}{\rightarrow} M^{\prime}(\pi) \rightarrow 0 \\
\downarrow & \stackrel{\hat{\pi}}{M^{0}} \stackrel{\downarrow}{\rightarrow} M(\pi)
\end{aligned}
$$

where the vertical arrows denote the canonical identifications. The first horizontal sequence is exact. On the other hand we can easily see that the transformation $\rho(\exp x)\left(x \in H(V)^{c}\right)$ is the translation by $x$ in $N^{0}=V+H(V)^{c}$. Thus we obtain

$$
\hat{\pi}^{-1}(\hat{\pi}(p))=p+\operatorname{Ker} \pi \quad p \in M^{0}
$$

while

$$
\tilde{\pi}^{-1}(\tilde{\pi}(p))=p+(\operatorname{Ker} \pi)^{c} \quad p \in M^{0} .
$$

Remark. In the next section, by identifying $M^{\prime}\left(\mathfrak{m}^{0}\right)$ with $M^{0}$ in canonical manner, we often regard $M^{0}$ as a group manifold. Then the left operation $L_{p}$ of $p\left(p \in M^{0}\right)$ can be uniquely extended to an affine transformation $\rho\left(p^{\prime}\right)$ of $N^{0}$ when $p^{\prime}$ is identified with $p$ under the identification.

Now let us prove our assertion stated before Proposition 1.2.1. Let $U$ be a domain of $M(\pi)$ and $f$ a continuous function on $U$ such 
that $\hat{\pi}^{*}(f)$ is the restriction of a holomorphic function $u^{\prime}$ on a domain $U^{\prime}$ in $N^{0}$ containing $\hat{\pi}^{-1}(U)$. Let $\tau_{h}(h \in H(V))$ denote the translation $x \rightarrow x+h\left(x \in N^{0}\right)$ and let $\partial_{h}$ be the vector field which generates 1parameter transformation group $\left(\tau_{t h}\right)_{t \in R}$. Note $\left.\left(\partial_{h} u^{\prime}\right)\right|_{\hat{\pi}^{-1}(S)}=\partial_{h} \hat{\pi}^{*}(f)=0$ for $h \in \operatorname{Ker} \pi$. Thus Proposition 1.2.1 implies

$$
\partial_{h} u^{\prime}=0 \quad h \in \operatorname{Ker} \pi \text {. }
$$

Note that $u^{\prime}$ is holomorphic. Hence it follows also

$$
\partial_{i h} u^{\prime}=0 \quad h \in \operatorname{Ker} \pi \text {. }
$$

Thus $u^{\prime}$ is constant on each component of the intersection of linear variety $p+(\operatorname{Ker} \pi)^{c}$ with $U^{\prime}$ for each $p \in U^{\prime}$. This implies that, for any continuous map $d$ of $V+\pi(H(V))^{c}$ into $N^{0}$ such that $\tilde{\pi} \circ d$ is the identity, the function $u=u^{\prime} \circ d$ on $d^{-}\left(U^{\prime}\right)$ is holomorphic. If, moreover, $d(M(\pi)) \subseteq M^{0}$, then $\left.u\right|_{v}=f$. Thus the only task is to give such a map $d$. But this can be obtained in the following way. Let $\sigma$ be a linear map of $W=\pi(H(V))$ into $H(V)$ such that $\pi \circ \sigma=i d_{W}$. Set

$$
d(x, y)=\left(x, \sigma^{c}\left(y+\frac{1}{4} i[x, I x]\right)-\frac{1}{4} i[x, I x]_{0}\right) \quad x \in V, y \in W^{c}
$$

where $[],,[,]_{0}$ are the bracket operations of $\mathfrak{m}(\pi) \mathfrak{m}_{0}$, respectively. It is evident from $[]=,\pi[,]_{0}$ that $d$ is the map with the desired properties.

Q.E.D.

In the next section, instead of $M^{0}$, we shall study an equivalent real submanifold $D_{0}$ which is the Šilow boundary of certain Siegel domain of the second kind. The equivalence of $D_{0}$ with $M^{0}$, though almost obvious, will be firstly showed in $\$ 5$.

\section{\$2. Holomorphic Hulls for Open Subsets of $D_{0}$}

This section is divided into two parts 2.1 and 2.2. The first half consists of long, rather tedious but important preparation for the second half. For this reason the motivation of the first half is not clear for the readers who want to know the meanings of notations, lemmas, propositions and so on. Here we indicate only that all of these are 
made to be fitted for the application to $D_{0}, D$ introduced at the beginning of the second half.

2.1. Basic Continuation Theorem. Fix an $n$-dimensional complex Hilbert space $V$ with the inner product $($,$) and denote by \mathcal{G}$ the vector space of linear operators of $V$ into itself. For $A \in \mathcal{G}$ we shall denote by $A^{*}$ its adjoint, i.e., $(A x, y)=\left(x, A^{*} y\right) x, y \in V$. As usual we call $A$ hermitian if $A=A^{*}$. For a hermitian operator $A, A>0$ (resp. $A \geq 0)$ means that $(A x, x)>0$ (res. $(A x, x) \geq 0$ ) for $0 \neq x \in V$. For $y \in V$ the mapping $x \rightarrow(x, y)$ is a linear form on $V$, which we denote by $y^{*}$. Then $x \otimes y^{*}$ can be regarded as an element of $\mathcal{G}$ since $V \otimes V^{*}$ can be canonical identified with $\mathcal{G}$. Further we denote by $l$ the mapping of $\mathcal{G} \times V$ into $\mathcal{G}$ defined by

$$
\mathfrak{l}(A, x)=\frac{1}{i}\left(A-A^{*}\right)-x \otimes x^{*} \quad A \in \mathcal{G} ; x \in V .
$$

$\mathfrak{l}(A, x)$ is obviously hermitian. Let $S_{1}$ denote the unit sphere of $V$ and $\Delta$ the unit disc in $C$, i.e.,

$$
\begin{gathered}
S_{1}=\{x \in V:\|x\|=1\} \\
\Delta=\{\zeta \in C ;|\zeta| \leq 1\}
\end{gathered}
$$

where $\|x\|^{1}=(x, x)$. We shall shortly denote by $\mathcal{E}$ the product space $\mathcal{G} \times V \times S_{1} \times(-\infty, \infty)$ and by $\mathcal{E}^{0}$ the open, dense subset $\{(A, x, y, r)$; $(\mathfrak{l}(A, x) y, y) \neq 0\}$ of $\mathcal{E}$. For later purposes we shall regard $A, x, y, r$ as the projections of $\mathcal{E}$ onto $\mathcal{G}, V, S_{1},(-\infty, \infty)$ respectively, i.e., $\varepsilon=(A(\varepsilon), x(\varepsilon), y(\varepsilon), r(\varepsilon))$ for $\varepsilon \in \mathcal{E}$, and further introduce some convenient notations setting

$$
\begin{aligned}
& \pi(\varepsilon)=(A(\varepsilon), x(\varepsilon)) \\
& \tilde{y}(\varepsilon)=l(\pi(\varepsilon)) y(\varepsilon)
\end{aligned} \quad \varepsilon \in \mathcal{E} .
$$

Define a continuous function $\lambda$ on $\mathcal{E}^{0}$ and a continuous mapping $\sigma$ of $\mathcal{E}^{0} \times \boldsymbol{C}$ into $\mathcal{G} \times V$ setting

$$
\begin{aligned}
\lambda(\varepsilon) & =\left(\mid\left(\left.l(\pi(\varepsilon) y(\varepsilon), y(\varepsilon))\right|^{-1}+r(\varepsilon)^{2}\right)^{1 / 2}\right. \\
& =\left(|(\tilde{y}(\varepsilon), y(\varepsilon))|^{-1}+r(\varepsilon)^{2}\right)^{1 / 2}
\end{aligned}
$$




$$
\begin{gathered}
\sigma(\varepsilon, \zeta)=\left(A(\varepsilon)+i(\lambda(\varepsilon) \zeta-r(\varepsilon)) y(\varepsilon) \otimes(x(\varepsilon)-r(\varepsilon) y(\varepsilon))^{*},\right. \\
x(\varepsilon)+(\lambda(\varepsilon) \zeta-r(\varepsilon)) y(\varepsilon)) .
\end{gathered}
$$

Then $\sigma$ has the following properties.

(1) $\sigma_{\varepsilon}$ is a holomorphic mapping of $C$ into $\mathcal{G} \times V$ where we denote by $\sigma \varepsilon$ the mapping $\Delta \ni \zeta \rightarrow \sigma \varepsilon(\zeta)=\sigma(\varepsilon, \zeta)$.

$$
\pi(\varepsilon) \in \sigma_{\varepsilon}(\Delta) \text {. }
$$

In fact $\pi(\varepsilon)=\sigma \varepsilon\left(\frac{r(\varepsilon)}{\lambda(\varepsilon)}\right)$ and $\left|\frac{r(\varepsilon)}{\lambda(\varepsilon)}\right|<1$. By simple computation we can obtain

$$
\begin{aligned}
& \mathfrak{l}(\sigma(\varepsilon, \zeta))=\mathfrak{l}(\pi(\varepsilon)) \\
& \quad-\left(|\zeta|^{2}|(j(\varepsilon), y(\varepsilon))|^{-1}+r(\varepsilon)^{2}\left(|\zeta|^{2}-1\right)\right) y(\varepsilon) \otimes y(\varepsilon)^{*} .
\end{aligned}
$$

In particular, if $|\zeta|=1$,

$$
\mathfrak{l}(\sigma(\varepsilon, \zeta))=\mathfrak{l}(\pi(\varepsilon))-|(y(\varepsilon), y(\varepsilon))|^{-1} \tilde{y}(\varepsilon) \otimes y(\varepsilon)^{*} .
$$

Set, for a subset $S$ of $\mathcal{G} \times V$,

$$
\begin{aligned}
\widetilde{E}^{0} S= & \left\{\varepsilon \in \mathcal{E}^{0} ; \sigma_{\varepsilon}(\partial \Delta) \subseteq S\right\} \quad E^{0} S=\pi\left(\widetilde{E}^{0} S\right) \\
& E S=S \cup E^{0} S .
\end{aligned}
$$

Then we have

$$
\begin{gathered}
S \subseteq T \Rightarrow E S \subseteq E T \quad \bigcup_{\lambda} E S_{\lambda} \subseteq E\left(\bigcup_{\lambda} S_{\lambda}\right) \\
E\left(\cap_{\lambda} S_{\lambda}\right) \subseteq \bigcap_{\lambda} E S_{\lambda} .
\end{gathered}
$$

Further, for a bounded set $S$,

$$
E S \subseteq \widehat{S}
$$

Here $\widehat{S}$ means $\widehat{S}_{\mathcal{Q}} \times V$. (5) follows from (1) and (2).

Lemma 2.1.1. If $O$ is an open set of $\mathcal{E} \times V$, then $E^{\circ} O, E O$ are open sets.

In fact $\widetilde{E}^{\circ} O$ is an open set in $\mathcal{E}^{\circ}$ by the continuity of $\sigma$ and $\pi$ is an open mapping $\mathcal{E}$ onto $\mathcal{G} \times V$. Therefore $E^{\circ} O=\pi\left(E^{\circ} O\right)$ is open.

Now we are in a position to study holomorphic extendibility from $S$ to $E S$ for a subset $S$ satisfying some conditions. Let $\varphi_{t}(t>0)$ be 
the holomorphic automorphism of $\mathcal{G} \times V$ defined by $\varphi_{t}(A, x)=\left(t^{2} A, t x\right)$. A subset $S$ of $G \times V$ is said to be $\varphi$-star-shaped if $S$ contains 0 and if $\varphi_{t} S \subseteq S$ for $0<t<1$. All $\varphi$-star-shaped sets are connected. If $S$ is $\varphi$-star-shaped, then $E S$ is $\varphi$-star-shaped as is evident by the following relation:

$$
\varphi_{t} \circ \sigma(A, x, y, r ; \zeta)=\sigma\left(t^{2} A, t x, y, t^{-1} r, \sigma\right)
$$

Now the key of our discussion is the following.

Proposition 2.1.1. Let $O$ be a $\varphi$-star-shaped open subset of $\mathcal{G} \times V$. Then, for any $f \in H(O)$, there exists uniquely $E f$ of $H(E O)$ such that $\left.E f\right|_{0}=f$.

For the proof of this we need some notation and lemmas. For a holomorphic function $f$ on $O$, we define a continuous function $\widetilde{E}^{\circ} f$ on $\widetilde{E}^{\circ} O$ setting

$$
\widetilde{E}^{\circ} f(\varepsilon)=\int_{0}^{2 \pi} f\left(\sigma_{\varepsilon}\left(e^{i \Phi}\right)\right) P(\varphi, r(\varepsilon) / \lambda(\varepsilon)) d \varphi
$$

where $P(\varphi, z)$ is the Poisson kernel for the unit disc in $C$, i.e.,

$$
P(\varphi, z)=\frac{1-|z|^{2}}{2 \pi\left|e^{i \Phi}-z\right|^{2}}
$$

Lemma 2.1.2. Suppose that $O$ is a bounded open set. If $f$ is the restriction to $O$ of a holomorphic function $u$ in a neighbourhood of $\hat{O}$, then

$$
\widetilde{E}^{\circ} f(\varepsilon)=u(\pi(\varepsilon)) .
$$

Proof is evident if one observes that, for any $\varepsilon \in E^{\circ} O$,

$$
\sigma_{\varepsilon}(\Delta) \subseteq \widehat{O}
$$

so that $f\left(\sigma_{\varepsilon}\left(e^{i \Phi}\right)\right)$ is the boundary value to $\partial \Delta$ of the holomorphic function $u\left(\sigma_{\varepsilon}(\zeta)\right)$ in a neighbourhood of $\Delta$.

To formulate next lemma we need a new notion: Let $\Omega$ be a $C^{\infty}$ manifold, $\mathscr{D}^{\prime}(\Omega)$ the space of distributions on $\Omega$. A mapping $t \rightarrow f(t)$ from an open interval $(a, b)$ into $\mathscr{D}^{\prime}(\Omega)$ is called an analytic $\mathscr{D}^{\prime}(\Omega)$ valued function on $(a, b)$ if, for any $C^{\infty}$ density $\psi$ with compact support, the function $\langle f(t), \psi\rangle$ is (real) analytic in $(a, b)$. 
Lemma 2.1.3. Let $P(D)$ be a scalar differential operator on $\Omega$ and $f(t)$ be an analytic $\mathcal{D}^{\prime}(\Omega)$-valued function on $(a, b)$. Suppose $P(D) f(t)=0$ for $t$ in a non-empty open subset of $(a, b)$. Then $P(D) f(t)=0$ for any $t \in(a, b)$.

Proof. Obvious.

Now define a function space on $E^{\circ} O$ setting

$$
\mathcal{L}=\left\{f \in C^{\infty}\left(\widetilde{E}^{\circ}\right) O ;{ }^{\prime} f^{\prime} \in H\left(E^{\circ} O\right)^{{ }_{\varepsilon}} \in \widetilde{E}^{\circ} O f^{\prime}(\pi(\varepsilon))=f(\varepsilon)\right\} .
$$

Lemma 2.1.4. Let $f(t)$ he a continuous $\mathscr{D}^{1}\left(\widetilde{E}^{\circ} O\right)$-valued function on $(0,1]$ a nalytic in $(0,1]$ such that, for $t$ in a non-empty open subset of $(0,1), f(t) \in \mathcal{L}$. Then $f(1) \in \mathcal{L}$.

Proof. This follows immediately from Lemma 6 since there exist scalar differential operators $P_{r}(D)(r=1,2, \cdots, m)$ on $E^{\circ} O$ such that $\mathcal{L}$ is the set of solutions in $\mathscr{D}^{\prime}\left(\widetilde{E}^{\circ} O\right)$ of the overdetermired elliptic system of differential equations

$$
P_{1}(D) v=0, \cdots, \quad P_{m}(D) v=0 .
$$

The construction of such $P_{r}(D)$ is easy and shall be left to the reader.

Now we are ready to prove Proposition 2.1.1.

Proof of Proposition 2.1.1. Any $\varphi$-star-shaped open set $O$ can be represented as the union of an ircreasing sequence of $\varphi$-star-shaped bounded open sets $O_{1}, O_{2}, \cdots$. It follows easily

$$
E O=\bigcap_{j} E O_{j}
$$

and each $E O_{j}$ is connected as was remarked before. Therefore we may assume that $O$ is bounded. For $z \in \boldsymbol{C}$, we denote by $\varphi_{z}$ the holomorphic mapping of $\mathcal{G} \times V$ into itself

$$
(A, x) \rightarrow\left(z^{2} A, z x\right) \text {. }
$$

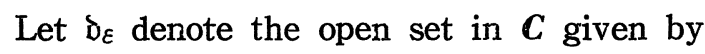

$$
\grave{D}_{\varepsilon}=\left\{z \in C: \varphi_{z} \circ \sigma_{\varepsilon}(\partial \Delta) \subseteq O\right\}
$$

and let $\psi$ be a $C^{\infty}$-function on $E^{\circ} O$ with compact support $K$. Then each $\grave{D}_{\varepsilon}(\varepsilon \in K)$ contains a fixed neighbourhood $\grave{D}_{K}$ of the $[0,1]$ since $\grave{D}_{\varepsilon}$ 
depends continuously on $\varepsilon$ and since $[0,1] \subseteq \searrow_{\varepsilon}\left(\varepsilon \in \widetilde{E}^{\circ} O\right)$. Define a continuous function $F^{\prime}$ on $\delta_{K} \times \widetilde{E}^{\circ} O \times \partial \Delta$

$$
F^{\prime}\left(z, \varepsilon, e^{i \varphi}\right)=\psi(\varepsilon) f \circ \varphi_{z} \circ \sigma_{\varepsilon}\left(e^{i \varphi}\right) P(\varphi \cdot r(\varepsilon) / \lambda(\varepsilon)) .
$$

Then $F^{\prime}$ is obviously holomorphic with respect to $z$ in $\delta_{K}$. Therefore, for a fixed volume $d \varepsilon$ on $\widetilde{E}^{\circ} O$,

$$
\int_{\tilde{E}^{\circ} O} F(z, \varepsilon) \psi(\varepsilon) d \varepsilon
$$

is also analytic in $\grave{D}_{K}$ when one puts

$$
F(z, \varepsilon)=\int_{0}^{2 \pi} f^{\circ} \psi_{z} \circ \sigma_{\varepsilon}\left(e^{i \rho}\right) P(\varphi, r(\varepsilon) / \lambda(\varepsilon)) d \varphi
$$

for $(z, \varepsilon)$ such that $z \in \grave{D}_{\varepsilon}$. In particular

$$
\int_{\widetilde{E}^{\circ} O} F(t, \varepsilon) \psi(\varepsilon) d \varepsilon
$$

is real analytic with respect to $t$ in $(0,1)$, thus the continuous $\mathscr{D}^{\prime}\left(E^{\circ} O\right)$-valued function

$$
t \rightarrow F(t, \cdot)
$$

on $[0,1]$ is analytic in $(0,1)$. Therefore in order to show $\widetilde{E}^{\circ} f=F(1, \cdot)$ $\in \mathcal{L}$, it suffices to prove that, for sufficiently small $t, F(t, \cdot)$ is in $\mathcal{L}$ (Lemma 2.1.4.).

For this purpose let $u_{t}(0<t \leq 1)$ denote the holomorphic function defined on $\varphi_{t}^{-1} O=\varphi_{1 / t} O$ setting

$$
u_{t}(p)=f\left(\varphi_{t}(p)\right) \quad p \in \varphi_{1 / t} O
$$

and $f_{t}$ the restriction to $O$ of $u t$. Then we have

$$
F(t, \varepsilon)=\widetilde{E}^{\circ} f_{t}(\varepsilon) \text {. }
$$

Therefore, for $t$ such that $\varphi_{1 / t} O$ contains $\widetilde{O}$, we have by Lemma 2.1.2,

$$
F(t, \varepsilon)=u_{t}(\pi(\varepsilon)) \text {. }
$$

But, for sufficiently small $t, \hat{O} \subseteq \varphi_{1 / t} O$ since $\hat{O}$ is bounded. Thus, for sufficiently small $t, F(t, \cdot) \in \mathcal{L}$ which proves that $\widetilde{E}^{\circ} f \in \mathcal{L}$. In other words there exists a holomorphic function $E^{\circ} f$ such that 


$$
\widetilde{E}^{\circ} f(\varepsilon)=E^{\circ} f(\pi(\varepsilon)) .
$$

Now what remains to be proved is that, in the intersection of $O$ and $E^{\circ} O$,

$$
E^{\circ} f=f .
$$

To show this we set, for $\varepsilon \in \widetilde{E}^{\circ} O$ such that $\pi(\varepsilon) \in O$,

$$
G(t)=F(t, \varepsilon)-f_{t}(\pi(\varepsilon)) .
$$

Then by (6) $G(t)$ vanishes when $t$ is sufficiently small. Since, moreover, $G(t)$ is real analytic in $t, G(1)=0$ and (7) is proved. Thus, if one defines a holomorphic function $E f$ on $E O$ setting

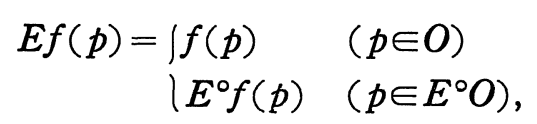

we obtain the desired holomorphic extession $E f$ of $f$. The uniqueness of $E f$ is obvious since $E O$ is conrected.

For any subset $S$ of $\mathcal{G} \times V$ let $H^{\circ}(S)$ denote the set of the restrictions to $S$ of holomorphic functions defined on some neighbouihood of $S$. Then in terms of $H^{\circ}(S)$ we can give a more convenient formulation to Proposition 2.1.1.

Corollary 2.1.1. If $S$ is a $\varphi$-star-shaped subset of $\mathcal{G} \times V$, then there exists a linear operator $E$ from $H^{\circ}(S)$ into $H^{\circ}(E S)$ such that

$$
\begin{gathered}
\left.E f\right|_{s}=f \\
\sup _{p \in E S}|E f(p)|=\sup _{p \in S}|f(p)|
\end{gathered}
$$

for $f \in H^{\circ}(S)$. In particular the restriction map $H^{\circ}(E S) \rightarrow H^{\circ}(S)$ is onto.

Proof. Suppose $f \in H^{\circ}(S)$. Then there exists an open subset $U \supseteq S$ of $\mathcal{G} \times V$ and a holomorphic function $g$ in $U$ such that $\left.g\right|_{s}=f$. Then the set

$$
O=\left\{p \in \mathcal{G} \times V: 0 \leq^{\forall} t \leq 1 \varphi_{t}(p) \in U\right\}
$$

is $\varphi$-star-shaped and open in $\mathcal{G} \times V$. Certainly $O$ contains $S$. Thus we 
can assume that $U=O$. But then $E g \in H^{\circ}(E O)$ and $E g \mid O=g$ in the notation of Proposition 2.1.1. Set $E f=\left.E g\right|_{E s}$. From the explicit construction of $E g, E f$ is given by the formula

$$
E f(\pi(\varepsilon))=\int_{0}^{2 \pi} f\left(\sigma_{\varepsilon}\left(e^{i \Phi}\right)\right) P(\varphi, x(\varepsilon) / \lambda(\varepsilon)) d \varphi \quad \varepsilon \in \widetilde{E}^{\circ} S .
$$

Thus it follows

$$
\sup _{p \in E S}|E f(p)|=\sup _{p \in S}|f(p)| .
$$

Q.E.D.

2.2. Application to $D_{0}$. From now on we shall apply the result obtained so far to the holomorphic continuation from the Silow boundary of the Siegel domain

$$
D=\{(A, x) \in \mathcal{G} \times V ; \mathfrak{l}(A, x)>0\} .
$$

Set for $k=0,1,2, \cdots$

$$
D_{k}=\{(A, x) ;=\mathfrak{l}(A, x) \geq 0, \text { rank of } \mathfrak{l}(A, x) \leq k\} .
$$

Each $D_{k}$ is a closed subset of $\mathcal{G} \times V . \quad D_{0}$ is the Šlow boundary of $D$ (See Piatetski-`̌apiro [7]). Further $\partial D=D_{n-1}, \bar{D}=D_{n}=D_{n+1}=\cdots$. Now set

$$
\mathcal{E}_{k}^{\circ}=\left(D_{k} \times S_{1} \times(-\infty, \infty)\right) \cap \mathcal{E}^{\circ} .
$$

Then we have

Lemma 2.2.1. Let $\varepsilon$ be an element of $\mathcal{E}^{\circ}$. Then $\varepsilon \in \mathcal{E}_{k}^{\circ}$ if and only if $\sigma_{\varepsilon}(\partial \Delta) \subseteq D_{k-1}$.

Proof. From (3)' it follows that $\sigma_{\varepsilon}(\partial \Delta) \subseteq D_{k-1}$ if and only if the rank of the hermitian endomorphism

$$
\mathcal{A}(\varepsilon)=\mathfrak{l}(\pi(\varepsilon))-|(y(\varepsilon), y(\varepsilon))|^{-1} y(\varepsilon) \otimes y(\varepsilon)^{*}
$$

is less than $k$ and $\mathcal{A}(\varepsilon) \geq 0$. Now suppose $\varepsilon \in \mathcal{E}_{k}^{\circ}$. Then $\mathfrak{l}(\pi(\varepsilon)) \geq 0$, rank of $\mathfrak{l}(\pi(\varepsilon)) \leq k$, and $(y(\varepsilon), y(\varepsilon)) \neq 0$. Hence $(y(\varepsilon), y(\varepsilon))=(\mathfrak{l}(\pi(\varepsilon))$ $y(\varepsilon), y(\varepsilon))>0$, and tilus

$$
\mathscr{A}(\varepsilon)=\mathfrak{l}(\pi(\varepsilon))-(y(\varepsilon), y(\varepsilon))^{-1} \tilde{y}(\varepsilon) \otimes y(\varepsilon)^{*} .
$$

Therefore $\mathcal{A}(\varepsilon) y(\varepsilon)=0$, while $l(\pi(\varepsilon) y(\varepsilon)=y(\varepsilon) \neq 0$. On the other hand $\mathfrak{I}(\pi(\varepsilon))-\mathcal{A}(\varepsilon)=(y(\varepsilon), y(\varepsilon))^{-1} y(\varepsilon) \otimes y(\varepsilon)^{*} \geq 0$. Thus, once $\mathcal{A}(\varepsilon) \geq 0$ is 
proved, it follows

i.e.,

$$
\text { rank of } \mathcal{A}(\varepsilon) \leq k-1 \text {, }
$$

$$
\sigma_{\varepsilon}(\partial \Delta) \subseteq D_{k-1} \cdot
$$

To show $\mathcal{A}(\varepsilon) \geq 0$ we shall first observe that

$$
(\mathfrak{l}(\pi(\varepsilon))(y(\varepsilon)+t x), y(\varepsilon)+t x) \geq 0
$$

for $t \in \boldsymbol{C}$. Hence we obtain

$$
(y(\varepsilon), y(\varepsilon))(\mathfrak{l}(\pi(\varepsilon)) x, x)-|(y(\varepsilon), x)|^{2} \geq 0 .
$$

But the left-hand side is equal to $(\tilde{y}(\varepsilon), y(\varepsilon))(\mathcal{A}(\varepsilon) x, x)$. Therefore

$$
(\mathcal{A}(\varepsilon) x, x) \geq 0 \quad x \in V .
$$

Thus $\mathcal{A}(\varepsilon) \geq 0$ and $\sigma_{\varepsilon}(\partial \Delta) \subseteq D_{k-1}$ is proved.

Conversely suppose that $A(\varepsilon) \geq 0$ and that rank of $A(\varepsilon) \leq k-1$. The image of $\mathfrak{l}(\pi(\varepsilon))$ is contained in the sum of the image of $\mathcal{A}(\varepsilon)$ and the $i$-dimensional subspace generated by $y(\varepsilon)$. Thus

$$
\text { rank of } \mathfrak{l}(\pi(\varepsilon)) \leq \text { rank of }(\mathcal{A}(\varepsilon)) \text { plus } 1 \leq k \text {. }
$$

On the other hand

$$
\mathfrak{l}(\pi(\varepsilon))=A(\varepsilon)+|(y(\varepsilon), y(\varepsilon))|^{-1} \tilde{y}(\varepsilon) \otimes \tilde{y}(\varepsilon)^{*} \geq A(\varepsilon) \geq 0 .
$$

Thus $\pi(\varepsilon) \in D_{k}$, that is, $\varepsilon \in \mathcal{E}_{k}^{\circ}$.

Q.E.D.

By this lemma the restriction $\sigma_{k}$ of $\sigma$ to the set $\mathcal{E}_{k}^{\circ} \times \partial \Delta$ is a continuous map of $\mathcal{E}_{k}^{\circ}$ into $D_{k-1}$. Further if $S$ is a subset of $D_{k-1}$, then $E^{\circ} S=\left\{\varepsilon \in \mathcal{E}_{k}^{\circ} ; \sigma_{\varepsilon}(\partial \Delta) \subseteq S\right\}$. Note that the restriction $\pi^{k}$ of $\pi$ to $\mathcal{E}_{k}^{\circ}$ is an open mapping from $\mathcal{E}_{k}^{\circ}$ onto $D_{k}$. Thus we obtain

Lemma 2.2.2. For an open subset $S$ of $D_{k-1}, E^{\circ} S$ is open in $D_{k}$.

Let $E_{k}$ denote the $k$-th power of the set operation $E$, that is, $E_{k} S$ is defined as follows:

$$
\begin{aligned}
& E_{0} S=S \\
& E_{r+1} S=E\left(E_{k} S\right) .
\end{aligned}
$$

Then we have

Lemma 2.2.3. $E_{k 11} S=S \cup E^{\circ}\left(E_{i} S\right)$ 
Proof. For $k=0$, there remains nothing to be proved. Assume that the case $k=j$ is proved. Then we have

$$
\begin{aligned}
E_{j+2} S & =E^{\circ}\left(E_{j+1} S\right) \cup E_{j+1} S \\
& =E^{\circ}\left(E_{j+1} S\right) \cup E^{\circ}\left(E_{j} S\right) \cup S \\
& =E^{\circ}\left(E_{j+1} S\right) \cup S
\end{aligned}
$$

since $E^{\circ}\left(E_{j} S\right) \subseteq E^{\circ}\left(E_{j+1} S\right)$. Thus the case $k=j+1$ is proved.

Q.E.D.

Proposition 2.2.1. If the subset $S$ of $D_{0}$ is open in $D_{0}$, then $E_{k} S$ is an open subset of $D_{k}$.

Proof. First we prove this proposition assuming the following lemma.

Lemma 2.2.4. Under the assumption of Proposition 2.2.1, $S$ is contained in the interior of $E_{k} S$.

Let us now prove Proposition 2.2.1. For $k=0$ nothing remains to be proved. Suppese that $E_{j} S$ is open in $D_{j}$. Then $E^{\circ}\left(E_{j} S\right)$ is open in $D_{j+1}$ by Lemma 2.2.2. But by Lemma 2.2.3. we have

$$
E_{j+1} S=S \cup E^{\circ}\left(E_{j} S\right) \text {. }
$$

Therefore by Lemma 2.2.4. $E_{j+1} S$ is open in $D_{j+1}$.

Q.E.D.

Before proving Lemma 2.2.4 we shall recall the group-theoretic aspect of $D_{0}$, which will facilitate the proof of Lemma 2.2.4. For a point $p$ of $D_{0}$ define an affine transformation $\rho(p)$ by setting

$$
\rho(p)\left(A^{\prime}, x^{\prime}\right)=\left(A+A^{\prime}+i x^{\prime} \otimes x^{*}, x+x^{\prime}\right)
$$

where $p=(A, x) \quad A \in \mathcal{G} x \in V$. By simple computation

$$
\mathfrak{l}(\rho(p) q)=\mathfrak{l}(p)+\mathfrak{l}(q)=\mathfrak{l}(q) \quad q \in \mathcal{G} \times V .
$$

Thus $\rho(p)$ leaves each $D_{j}$ invariant. In particular $\rho(p) p^{\prime} \in D_{0}$ for $p, p^{\prime} \in D_{0}$, so we can define a multiplication of a pair of elements of $D_{0}$ setting

$$
p \cdot p^{\prime}=\rho(p) p^{\prime} .
$$

This multiplication is associative 


$$
\left(p \cdot p^{\prime}\right) \cdot p^{\prime \prime}=p \cdot\left(p^{\prime} \cdot p^{\prime \prime}\right) \text {. }
$$

This follows immediately from

$$
x^{\prime} \otimes x^{*}+x^{\prime \prime} \otimes\left(x+x^{\prime}\right)^{*}=x^{\prime \prime} \otimes\left(x^{\prime}\right)^{*}+\left(x^{\prime}+x^{\prime \prime}\right) \otimes x^{*} .
$$

If $(A, x) \in D_{0}$, then $\left(-A+i x \otimes x^{*},-x\right) \in D_{0}$ and

$$
(A, x) \cdot\left(-A+i x \otimes x^{*},-x\right)=0 .
$$

Further $p \cdot 0=0 \cdot p=p$. Therefore $D_{0}$ is a Lie group with this multiplication. The map $D_{0} \ni p \rightarrow \rho(p) \in A F_{c}(\mathcal{G} \times V)$ is a Lie group isomorphism into. The group $\rho\left(D_{0}\right)$ operates transitively on $D_{0}$. Further we have

$$
E(\rho(p) S)=\rho(p) \cdot E S \text {. }
$$

In fact

$$
\sigma(\rho(p) q, y, r ; \zeta)=\rho(p) \sigma(q, y, r ; \zeta) .
$$

Thus, in order to prove Lemma 2.2.4, we may assume that $S \ni 0$ and it suffices to show that 0 is in the interior of $E_{k} S$ in $D_{k}$.

Proof of Lemma 2.2.4. First we shall introduce a norm on $\mathcal{G} \times V$ setting:

$$
\|p\|=\|A\|+\|x\|
$$

where $p=(A, x)$ and $\|A\|$ denotes the operator norm of $A$. By $B_{\delta}$ we denote the open ball of radius $\delta$ in $\mathcal{G} \times V$ :

$$
B_{\delta}=\{p \in \mathcal{G} \times V ;\|p\|<\delta\} .
$$

Suppcse that $p \in \bar{D},\|p\|<1$. Then $\|\mathfrak{l}(p)\| \leq 2\left(\|p\|+\|p\|^{2}\right) \leq 4\|p\|$. Therefore $\|\lambda(p, y, 0) \mathfrak{l}(p) y\|=\|\mathfrak{l}(p) y\| /\left\|\mathfrak{l}(p)^{1 / 2} y\right\| \leq\left\|\mathfrak{l}(p)^{1 / 2}\right\| \leq 2\|p\|^{1 / 2}$, hence for $\zeta \in \Delta$

$$
\begin{gathered}
\|\sigma(p, y, 0 ; \zeta)\| \leq\|p\|+\|\lambda(p, y, 0) l(p) y\|(\|x\|+1) \\
\leq\|p\|+2\|p\|^{1 / 2}(\|p\|+1) \leq 5\|p\|^{1 / 2} .
\end{gathered}
$$

Now suppose that $p \in\left(B_{(\delta / 5)^{2}} \cap D_{j+1}\right) \backslash D_{0}(\delta<1)$. Then there exists $y \in S_{1}$ such that $(\mathfrak{l}(p) y, y)>0$ which implies $\sigma(p, y, 0, \partial \Delta) \subset D_{j}$. But by (8) we have $\sigma(p, y, 0 ; \partial \Delta) \subset B_{\delta}$. Thus $p \in E^{\circ}\left(B_{\delta} \cap D_{j}\right)$. Hence $B_{(\delta / 5)^{2}} \cap D_{j+1} \backslash$ $D_{0} \subset E^{\circ}\left(B_{\delta} \cap D_{j}\right)$. Since $B_{(\delta / 5)^{2}} \cap D_{0} \subset B_{\delta} \cap D_{j} \subset E\left(B_{\delta} \cap D_{j}\right)$, we have

$$
B_{(\delta / 5) 2} \cap D_{j+1} \subset E\left(B_{\delta} \cap D_{j}\right) \text {. }
$$


Choose a positive number $\eta<1$ such that

$$
B_{\eta} \cap D_{0} \subset S \text {. }
$$

Then we have by (10)

$$
B_{(\eta / 5)^{3 k}} \cap D_{k} \subset E_{k} S,
$$

which implies that 0 is the interior of $E_{k} S$.

Q.E.D.

For a $\sigma$-compact subset $S$ of $\mathcal{G} \times V$ we shall also denote by $H(S)$ the closure of $H^{\circ}(S)$ in $C(S)$. Recall that any open subset of $D_{0}$ is generic (Proposition 1.2.1). Trerefcre by repeated use of Corollary 2.1.1 we obtain

Lemma 2.2.5. Let $S$ be a $\varphi$-star-shaped open subset of $D_{0}$. Then the restriction map $H\left(E_{n} S\right) \rightarrow H(S)$ is a topological isomorphism onto $H(S)$.

Proof. We stall first prove this assuming that $S$ is bounded. Set $S_{t}=\varphi_{t} S \quad 0<t<1$. Then $S_{t^{\prime}}$ is relatively compact in $S_{t^{\prime \prime}}$ when $t^{\prime}<t^{\prime \prime}$. Sirce $\varphi_{t} E_{n} S=E_{n} S_{t}, \quad E_{n} S_{t^{\prime}}$ is also relatively compact in $E_{n} S_{t^{\prime \prime}}$ if $t^{\prime}<t^{\prime \prime}$. Now, for $f \in H^{\circ}(S)$ we defire $E_{k} f \in H^{\circ}\left(E_{k} S\right)$ by irduction;

$$
\begin{aligned}
& E_{0} f=f \\
& E_{k} f=E\left(E_{k-1} f\right) \quad(k \geq 1) .
\end{aligned}
$$

Here $E$ is the lirear map given in Corollary 2.1.1. According to the estimate in this corollary,

$$
\sup _{p \in S_{t}}|f(p)|=\sup _{p \in E_{n} s_{t}}\left|E_{k} f(p)\right| \quad 0<t \leq 1 .
$$

Thus the map $f \rightarrow E_{n} f$ is a topological isomorphism of $H^{\circ}(S)$ into $H^{\circ}\left(E_{n} S\right)$. This is also onto sirce the restriction map $H^{\circ}\left(E_{n} S\right) \rightarrow H^{\circ}(S)$ is ore-to-ore by Prcpcsition 1.2.1. The map $E_{n}$ is thus extended to a unique topological isomorpl.ism of $H(S)$ onto $H\left(E_{n} S\right)$. But the inverse of $E_{n}$ is the restriction map, and we have thus obtaired the desired conclusion when $S$ is bounded.

In case $S$ is not bounded we set for $k=1,2, \cdots$

$$
S^{k}=S \cap\left\{(A, x) \in \mathcal{G} \times V ;\|A\|+\|x\|^{2}<k\right\} .
$$


Then it follows easily $E_{n} S=\bigcup_{k} E_{n} S^{k}$. Put

$$
S^{(k)}=\varphi_{(k-1) / k}\left(S^{k}\right) \quad(k=1,2, \cdots) .
$$

Then it also holds $E_{n} S=\bigcup_{k} E_{n} S^{(k)}$. On the other hand

$$
\overline{E_{n} S^{(k)}}=\overline{\varphi_{(k-1) / k} E_{n} S_{k}} \subset \varphi_{k /(k+1)} E_{n} S^{k} \subset \varphi_{k /(k+1)} E_{n} S^{k+1}=E_{n} S^{(k+1)} .
$$

Thus $E_{n} S^{(k)}$ is relatively compact in $E_{n} S^{(k+1)}$. Here $S^{(k)}$ is obviously bounded, and we have thus reduced the second case to the first.

Q.E.D.

By lemmas 2.2.4 and 2.2.5 we can improve Proposition 1.2.1 for standard real submanifold $D_{0}$ as follows.

Lemma 2.2.6. Let $U$ be a domain in $\mathcal{G} \times V$ such that $U \cap D_{0} \neq \phi$. Then a continuous function $u$ on $U \cap \bar{D}$ such that $\left.u\right|_{U \cap D_{0}}=0$ and $\left.u\right|_{U_{1 \cap D}} \in H(U \cap D)$ must vanish identically in $U \cap \bar{D}$.

Proof. Since $\rho\left(D_{0}\right)$ operates transitively on $D_{0}$, we may assume that $U \cap D_{0} \ni 0$. Take a sufficiently small $\delta>0$ so that $\bar{B}_{\delta} \subset U$ and set $S=B_{\delta} \cap D_{0}$. Then we have

$$
E S \subset \widehat{S} \subset \widehat{B_{\delta}}=\bar{B}_{\delta}
$$

By repeated use of this argument we obtain

$$
E_{n} S \subset \bar{B}_{\delta}
$$

and hence

$$
E_{n} S \subset \bar{B}_{\delta} \cap \bar{D}
$$

Since $\bar{B}_{\delta} \subset U$, there exists a positive number $\eta$ such that

$$
\bar{B}_{\delta}+i t I_{V} \subset U \quad 0 \leq t \leq \eta
$$

where $I_{V}$ denotes the identity map of $V$. Therefore, for $0<t \leq \eta$,

$$
E_{n} S+i t I_{V} \subset\left(\bar{B}_{\delta}+i t I_{V}\right) \cap\left(\bar{D}+i t I_{v}\right) \subset U \cap D,
$$

hence we obtain a continuous family $u_{t}(0<t<\eta)$ of elements of $H^{\circ}\left(E_{n} S\right)$ setting

$$
u_{t}(A, x)=u\left(A+i t I_{v}, x\right)
$$

for $(A, x) \in E_{n} S$. 
Certainly $u_{t} \rightarrow v$ at $t \rightarrow 0$ where we have set $v=\left.u\right|_{E_{n} s}$. Thus $v \in H\left(E_{n} S\right)$. But the restriction map $H\left(E_{n} S\right) \rightarrow H(S)$ is one-to-one by Lemma 2.2.5. Thus $v=0$ which implies $u=0$ since $E_{n} S$ is an open subset of $\bar{D}$.

Q.E.D.

Lemma 2.2.6 is a very powerful device for piecing together local extensiors into a global extension as will be explained in the following. Before proceeding we shall inticduce some function spaces and sheaves.

The system $(H(S))_{s: \text { ope. in } D_{0}}$ together with restriction maps forms a preskeaf on $D_{0}$ and we dencte by $\mathscr{H}$ the sheaf induced from this presheaf. For any open subset $S$ of $D_{0}, H(S)$ is defined by

$$
\begin{aligned}
& H(S)=\left\{f \in C(S):\left.f\right|_{s^{\prime}} \in H\left(S^{\prime}\right)\right. \text { for any relatively } \\
& \left.\quad \text { compact open subset } S^{\prime} \text { of } S\right\} .
\end{aligned}
$$

A continuous function $f$ on $S$ is $D$-holomorphic in $S$ if there exists an open sulset $U$ of $\mathcal{G} \times V$ containing $S$ and a continuous function $u$ on $U \cap \bar{D}$ such that (1) $\left.u\right|_{s}=f$ (2) $\left.u\right|_{U}{ }_{D}$ is holomorphic. We denote by $H_{D}(S)$ the set of $D$-holcmorphic furctions on $S$ and by $\mathscr{H}_{D}$ the sheaf of germs of $D$-holomorphic functiors. Further for an open set $V$ of $\bar{D}$ we denote by $\widetilde{H}(V)$ the subspace of $C(V)$

$$
\widetilde{H}(V)=\left\{u \in C(V) ;\left.u\right|_{V^{i} D} \text { is holomorphic }\right\} \text {. }
$$

As an immediate consequences of Lemma 2.2.5 we obtain

Lemma 2.2.7. $H(S) \subset \Gamma_{s}\left(\mathscr{H}_{D}\right)$ for an open set $S$ of $D_{0}$.

For the proof one may note that the system $I=\left\{\rho(p)\left(B_{\delta} \cap D_{0}\right)\right.$; $\left.p \in D_{0}, \delta>0\right\}$ is a fundamental system of neighbourhoods of $D_{0}$ and that each element of $I$ is an image by $\rho(p)$ of a $\varphi$-star-shaped neighkourhocd of $D_{0}$.

Lemma 2.2.8. $\Gamma_{s}\left(\mathscr{H}_{D}\right)=H_{D}(S)$ for an open set $S$ of $D_{0}$.

To prove this we need a special reighbourhocd system in $\bar{D}$ of a poir.t of $D_{0}$. Let $h(z)(z \in \mathcal{G})$ desote hermitia.s part of $z$, i.e.

$$
h(z)=\frac{1}{2}\left(z+z^{*}\right)
$$

ard $C_{\delta, \eta}\left(z_{0}, x_{0}\right)(\delta, \eta>0)$ the neighbourhood of $\left(z_{0}, x_{0}\right) \in D_{0}$ given by 


$$
\begin{aligned}
C_{\delta, \eta}\left(z_{0}, x_{0}\right)= & \left\{\left(z,{ }^{\prime} x\right) \in \bar{D} ;\left\|h\left(z-z_{0}\right)\right\|<\delta,\right. \\
& \left.\left\|x-x_{0}\right\|<\delta,\|\mathfrak{l}(z, x)\| \leq \eta\right\} .
\end{aligned}
$$

We also denote by $C_{\delta, 0}$ the intersection of $C_{\delta, \eta}$ and $D_{0}$ (this is obviously independent of $\eta$ ). If $0 \leq \delta \leq \delta^{\prime} \quad 0 \leq \eta \leq \eta^{\prime}$, it holds

$$
C_{\delta, \eta}\left(z_{0}, x_{0}\right) \subset C_{\delta^{\prime} \eta_{1}^{\prime}}\left(z_{0}, x_{0}\right)
$$

and $\left(C_{\delta, \eta}\left(z_{0}, x_{0}\right)\right)_{\delta, \eta>0}$ is a complete system of neighbourhoods of $\left(z_{0}, x_{0}\right)$.

Now consider the retracting deformation $\phi_{t}(0 \leq t \leq 1)$ of $\bar{D}$ onto $D_{0}$ given by

$$
\phi_{t}(z, x)=\left(z-\frac{i t}{2} \mathfrak{l}(z, x), x\right)
$$

Then

$$
\begin{aligned}
& \mathfrak{l}\left(\phi_{t}(z, x)\right)=(1-t) \mathfrak{l}(z, x) \\
& h\left(z-\frac{i t}{2} \mathfrak{l}(z, x)\right)=h(z) .
\end{aligned}
$$

Therefore, for $0 \leq t \leq 1$,

$$
\phi_{t} C_{\delta, \eta}\left(z_{0}, x_{0}\right)=C_{\delta,(1-t){ }_{1}}\left(z_{0}, x_{0}\right) \text {. }
$$

In particular, this implies that $C_{\delta, 0}\left(z_{0}, x_{0}\right) \cap C_{\delta^{\prime}, 0}\left(z_{1}, x_{1}\right) \neq \phi$ and $C_{\delta, \eta}\left(z_{0}, x^{0}\right)$ $\cap C_{\delta^{\prime}, \eta^{\prime}}\left(z_{1}, x_{1}\right)$ is connected whenever $C_{\delta, \eta}\left(z_{0}, x_{0}\right) \cap C_{\delta^{\prime}, \eta_{1}^{\prime}}\left(z_{1}, x_{1}\right) \neq \phi$. In fact $C_{\delta, 0}\left(z_{0}, x_{0}\right) \cap C_{\delta^{\prime}, 0}\left(z_{1}, x_{1}\right)$ is the deformation retract by $\phi_{t}$ of $C_{\delta, \eta}\left(z_{0}, x_{0}\right) \cap C_{\delta^{\prime},,^{\prime}}\left(z_{1}, x_{1}\right)$ and $C_{\delta}\left(z_{0}, x_{0}\right) \cap C_{\delta^{\prime}, 0}\left(z_{1}, x_{1}\right)$ is connected. The latter fact can be seen as follows; first consider the diffeomorphism $\varphi$ of $D_{0}$ onto $\mathcal{G}_{h} \times V$ given by

$$
\varphi(z, x)=(h(z), x)
$$

where $\mathcal{G}_{h}$ is the vector space of hermitian endomorphisms. Then

$$
\varphi\left(C_{\delta, 0}\left(z_{0}, x_{0}\right)\right)=\left\{(z, x) \in \mathcal{G}_{h} \times V:\left\|z-h\left(z_{0}\right)\right\|<\delta,\left\|x-x_{0}\right\|<\delta\right\}
$$

which is evidently convex and $\varphi\left(C_{\delta^{\prime}, 0}\left(z_{1}, x_{1}\right)\right)$ is also convex hence $\varphi\left(C_{\delta, 0}\left(z_{0}, x_{0}\right) \cap C_{\delta^{\prime}, 0}\left(z_{1}, x_{1}\right)\right)$ is convex, a fortiori, it is connected.

Now we shall turn to the proof of Lemma 2.2.8.

Proof of Lemma 2.2.8. Suppose that $f \in \Gamma_{S}\left(\mathcal{H}_{D}\right)$. Then for any point $p$ of $S$ one can choose a neighbourhood $V(p)$ in $\bar{D}$ of $p$ and an element $u_{p}$ of $\widetilde{H}(V(p))$ such that $\left.u_{p}\right|_{V(p) \backslash s}=\left.f\right|_{V(p)\urcorner s}$. We may assume 
that $V(p)=C_{\delta, \eta}(p)$ for some $\delta, \eta>0$ and that $V(p) \cap D_{0} \subset S$. We claim that

$$
V(p) \cap V\left(p^{\prime}\right) \neq\left.\phi \rightarrow u_{p}\right|_{v(p)\urcorner v\left(p^{\prime}\right)}=\left.u_{p^{\prime}}\right|_{v(p)} v\left(p^{\prime}\right) .
$$

As shown before, $V(p) \cap V\left(p^{\prime}\right) \cap D_{0}$ is non-empty if $V(p) \cap V\left(p^{\prime}\right) \neq \phi$. But $u_{p}-\left.u_{p^{\prime}}\right|_{v(p)} v\left(p^{\prime}\right)-D_{0}=f-f=0$. Therefore we have by Lemma 2.2.6

$$
u_{p}=u_{p^{\prime}} \text { in } V(p) \cap V\left(p^{\prime}\right)
$$

since $V(p) \cap V\left(p^{\prime}\right)$ is connected. Thus our claim is proved. Thus if we define a continuous function $u$ on $\bigcup_{p \subseteq S} V(p)$ by setting

$$
z \in V(p) \rightarrow u(z)=u_{p}(z),
$$

then $u$ is well-defined and $\left.u\right|_{s}=f$ and $u$ is holomorphic in $\bigcup_{p \in S} V(p) \cap D$. Thus $f \in H_{D}(S)$.

Q.E.D.

Lemma 2.2.9. $H_{D}(S) \subset \underset{\leftarrow}{H}(S)$ for an open set $S$ of $D_{0}$.

Proof. Let $S^{\prime}$ be a relatively compact open subset of $S$ and $U$ an open subset of $\mathcal{G} \times V$ containing $S$. Suppose further that $u \in \widetilde{H}(U \cap \bar{D})$. Then there exists a positive number $\eta$ such that

$$
S^{\prime}+i t I_{V} \subset U \text { for } 0 \leq t \leq \eta
$$

since $\bar{S}^{\prime} \subset S \subset U$. On the other hand if $t>0$,

$$
S^{\prime}+i t I_{V} \subset D_{0}+i t I_{V} \subset D \text {. }
$$

Therefore $\quad S^{\prime}+i t I_{V} \subset U \cap D(t>0)$.

Define a family $f_{t}(0<t \leq \eta)$ of elements of $H^{\circ}(S)$ setting

$$
f_{t}(p)=u\left(p+i t I_{V}\right) \quad p \in S^{\prime}
$$

Then $f_{t}$ is well-defined and $f_{t} \rightarrow f=\left.u\right|_{s^{\prime}}$ as $t \rightarrow 0$. Thus $\left.u\right|_{s} \in \underset{H}{H}(S)$.

$$
\text { Q.E.D. }
$$

In view of this lemma the natural restriction map

$$
\widetilde{H}(U) \rightarrow \underset{H}{ }\left(U \cap D_{0}\right)
$$

is well defined when $U$ is an open subset of $\bar{D}$ such that $U \cap D_{0} \neq \phi$. For any open subset $S$ of $D_{0}$ we shall now assign an open subset $U_{1}(S)$ of $\bar{D}$ such that $S \subseteq U_{1}(S)$ and such that the restriction map 


$$
\widetilde{H}\left(U_{1}(S)\right) \rightarrow H(S)
$$

is onto, that is,

$$
H_{D}(S)=H(S)=\left.H\left(U_{1}(S)\right)\right|_{s} .
$$

First we set for $\delta>0$

$$
Q_{\delta}=\left\{(A, x) \in D_{0} ;\|A\|+\|x\|^{2}<\delta^{2}\right\} .
$$

Then $Q_{\delta}$ is a $\varphi$-star-shaped neighbourhocd of 0 in $D_{0}$. In fact

$$
\varphi_{t} Q_{\delta}=Q_{t \delta} .
$$

Let $S$ be an open subset of $D_{0}$. Put

$$
\begin{aligned}
& \eta(p, S)=\sup \left\{\eta>0 ; \rho(p)\left(Q_{2 \eta}\right) \subseteq S\right\} \\
& Q(p, S)=\rho(p)\left(Q_{S(p . s)}\right) .
\end{aligned}
$$

Then $Q(p, S)$ is a relatively compact open subset of $S$ unless $S=D_{0}$. Hence, for $f \in H(S)$, there exists $u_{p}^{\prime} \in H\left(E_{n} Q(p, S)\right)$ such that

$$
\left.u_{p}^{\prime}\right|_{Q(p, S)}=\left.f\right|_{Q(p, S)}
$$

by Lemma 2.2.5. Now denote by $\delta^{\prime}(p, S)$ the supremum of the set

and put

$$
\left\{\delta>0 ; C_{2 \delta, 2 \delta}(p) \subset E_{n} Q(p, S)\right\}
$$

$$
\begin{aligned}
& V_{1}(p, S)=C_{\delta^{\prime}(p, s), \delta^{\prime}(p, s)}(p) \\
& U_{1}(S)=\bigcup_{p \in S} V_{1}(p, S) .
\end{aligned}
$$

Then certainly $U_{1}(S)$ is an open subset of $\bar{D}$ such that $U_{1}(S) \cap D_{0}=S$. Further is holds

$$
\left.u_{p}\right|_{V_{1}(p, s) \quad D_{0}}=\left.f\right|_{V_{1}(p, s)} D_{0}
$$

where we have set $u_{p}=\left.u_{p}^{\prime}\right|_{V_{1}(p, s)}$. Ey the same reasoning as in the proof of Lemma 2.2.8 there exists $u \in \widetilde{H}\left(U_{1}(S)\right)$ such that $\left.u\right|_{V_{1}(p, s)}=u_{p}$. Of course $f=\left.u\right|_{s}$ and we conclude

$$
\widetilde{H}(S)=\left.H\left(U_{1}(S)\right)\right|_{s},
$$

which, together with Lemmas 2.2.7-2.2.9, implies

$$
H(S)=\Gamma_{s}\left(\mathscr{H}_{D}\right)=H_{D}(S)=\left.\widetilde{H}\left(U_{1}(S)\right)\right|_{s} .
$$

From the first identity it follows also $\mathscr{H}=\mathscr{H}_{p}$. 
To sum up, we have thus proved

Theorem 2.2.1. For any open subset $S$ of $D_{0}$

$$
H(S)=\Gamma_{S}(\mathscr{H})\left(=\Gamma_{S}\left(\mathscr{H}_{D}\right)=H_{D}(S)\right)=\left.\widetilde{H}\left(U_{1}(S)\right)\right|_{s}
$$

Here $U_{1}(S)$ is open in $\bar{D}$.

This is the main theorem of this section. However, in later discussion (especially in $\S 5$ ), it will be required to exploit another open subset with certain additional property instead of $U_{1}(S)$. The rest we shall devote to the preparation for $\S 5$. In the above notation we set for $p \in S$

$$
\begin{aligned}
& \delta(p, S)=\min \left(\frac{1}{2} \delta^{\prime}(p, S), 1\right) \\
& V(p, S)=C_{\delta(p, s), \delta(p, s)}(p) \\
& U(S)=\bigcup_{p \in S} V(p, S) .
\end{aligned}
$$

$U(S)$ is an open subset of $\bar{D}$ such that $S \subseteq U(S) \subseteq U_{1}(S)$, and we have

Lemma 2.2.10. For any open subset $S$ of $D_{0}$

$$
\overline{U(S)} \backslash \partial S \subseteq U_{1}(S) \text {. }
$$

Proof. First we shall prove that $\delta(p, S)$ is continuous with respect to $p \in S$. The continuity of $\eta(p, S)$ is obvious. Note that

$$
\begin{aligned}
Q(p, S) & =\rho(p) Q_{r(p, s)} \\
& =\rho(p) \varphi_{\eta(p, s)}\left(Q_{1}\right) .
\end{aligned}
$$

Thus the relation

$$
C_{\delta, \delta}(p) \subseteq E_{n} Q(p, S)
$$

is equivalent to

$$
\varphi_{\eta(p, s)-1} \rho\left(p^{-1}\right) C_{\delta, \delta}(p) \subseteq E_{n} Q_{1} .
$$

The left hand side depends continuously on $p$ and $\delta$, while the right hand side is the definite open subset of $\bar{D}$. Thus $\delta^{\prime}(p, S)$ is continuous by its definition, and hence also $\delta(p, S)$ is continuous.

Next we prove that

$$
q \in V(p, S) \Rightarrow d(p) \leq 3(d(q)+2)
$$


where we have set $d(A, x)=\|A\|+\|x\|^{2}$ for $(A, x) \in \mathcal{G} \times V$. Set $p=(A, x), q=(B, y)$. Since $\delta(p, S) \leq 1$, we have

$$
\|h(A-B)\|<1,\|\mathfrak{l}(q)\| \leq 1,\|x-y\| \leq 1
$$

if $q \in V(p, S)$. Therefore

$$
\begin{aligned}
\|A\| & =\left\|h(A)+\frac{1}{2}\left(\mathfrak{I}(q)+i x \otimes x^{*}\right)\right\| \leq\|h(A)\|+\frac{1}{2}\|x\|^{2} \\
& \leq\|h(B)\|+\|y\|^{2}+\|h(A-B)\|+\|x-y\|^{2} \\
& \leq\|B\|+\|y\|^{2}+2, \\
\|x\|^{2} & \leq 2\left(\|y\|^{2}+1\right) .
\end{aligned}
$$

Thus it follows

$$
d(p) \leq 3(d(q)+2) .
$$

Now suppose $q_{0} \in \overline{U \backslash \partial S}$ and let $\left\{q_{v}\right\}_{\nu=1}^{\infty}$ be a sequence from $U(S)$ such that $q_{\nu} \rightarrow q_{0}(\nu \rightarrow \infty)$. Since $U(S)=\bigcup_{p \in S} V(p, S)$, there exists a sequence $\left\{p_{\nu}\right\}_{\nu=1}^{\infty} \subseteq S$ such that

$$
q_{\nu} \in V\left(p_{\nu}, S\right) .
$$

But then the inequality proved above implies that $\left\{p_{\nu}\right\}_{\nu=1}^{\infty}$ is bounded. Therefore we may assume that $p_{\nu}$ converges to $p_{0} \in \bar{S}$ taking a suitable subsequence if necessary. However $p_{0} \in \partial S$ contradicts $q_{0} \notin \partial S$, for one can see immediately $\delta^{\prime}\left(p_{\nu}, S\right) \rightarrow 0$ when $p_{\nu} \rightarrow p_{0} \in \partial S$. Thus $p_{0}$ must lie in $S$. Hence the definition of $V(p, S)$ and the continuity of $\delta(p, S)$ imply that

$$
q_{0} \in \overline{V\left(p_{0}, S\right)} \text {. }
$$

The right-hand side is contained in $V_{1}\left(p_{0}, S\right)$ since $\delta(p, S)$ $\leq \frac{1}{2} \delta^{\prime}(p, S)$. Therefore $q_{0} \in U_{1}(S)$. Thus we have proved

$$
\overline{U(S)} \backslash \partial S \subseteq U_{1}(S) . \quad \text { Q.E.D. }
$$

The next lemma will also be used in $\$ 5$.

Lemma 2.2.11. Let $H$ be a subspace of $\mathcal{G}_{n}$. Suppose that an open subset $S$ of $D_{0}$ and $f \in \Gamma_{\mathrm{S}}(\mathcal{H})$ are invariant by translations paallrel to $H$. Then $f$ can be extended into a function $u$ on $U_{1}(S)$ $+H^{c}$ so that $u_{U_{1}(s)} \in \widetilde{H}\left(U_{1}(S)\right)$ and $u$ is invariant by translations parallel to $H^{c} . U_{1}(S), U(S)$ are invariant by translations parallel to $H$. 
Proof. The second assertion follows immediately from

$$
C_{\delta, \eta}(p+h)=C_{\delta, \eta}(p)+h \quad h \in \mathcal{G}_{h} .
$$

To prove the first assertion we shall first show that the intersection of the line

$$
L_{k}(p)=\{p+i t h ; t \in \boldsymbol{R}\} \quad h \in \mathcal{G}_{k}
$$

with $U(S)$ is connected. From definition of $C_{\delta, \eta}(q)$ it follows that $L_{k}(p) \cap C_{\delta, \eta}(q)$ is either empty or

$$
\{p+i t h ; \mathfrak{l}(p)+2 t h \geq 0,\|\mathfrak{l}(p)+2 t h\|<\eta, \quad t \in \boldsymbol{R}\} .
$$

Thus $U_{1}(S) \cap L_{k}(p)=\{p+i t h ; \mathfrak{l}(p)+2 t h \geq 0,\|\mathfrak{l}(p)+2 t h\|<\bar{\eta}, \quad t \in \boldsymbol{R}\}$ where $\bar{\eta}=\sup \left\{\delta^{\prime}(q, S): L_{k}(p) \cap V_{1}(q, S) \neq \phi\right\}$. This is obviously con nected.

Let us now prove the existence of $u$ with the required property. From Theorem 2.2.1 it follows that there exists $u^{\prime} \in H\left(U_{1}(S)\right)$ such that $\left.u^{\prime}\right|_{s}=f$. However, by assumption

$$
\begin{aligned}
& \tau_{h}\left(U_{1}(S)\right)=U_{1}(S) \\
& \tau_{h}^{*}(f)=f
\end{aligned}
$$

where $h \in H$ and $\tau_{h}$ is the translation $p \rightarrow p+h$. Thus Lemma 2.2.6 implies

$$
\tau_{h}^{*}\left(u^{\prime}\right)=u^{\prime} \quad h \in H .
$$

Since $u^{\prime}$ is holomorphic in $U_{1}(S) \cap D$ and since $L_{k}(p) \cap U_{1}(S)$ is connected, we obtain for $h \in H$

$$
u^{\prime}(p+i h)=u^{\prime}(p) \text { if } p \in U_{1}(S) \cap D, p+i h \in U_{1}(S) .
$$

Now suppose only $p, p+i t h \in U_{1}(S)$. Choose a positive definite $h_{0} \in \mathcal{G}_{h}$. Since $U_{1}(S)$ is an open subset of $D$, there exists $\delta>0$ such that

$$
\begin{aligned}
& p(s)=p+i s h_{0} \in U_{1}(S) \\
& p(s)+i h \in U_{1}(S)
\end{aligned}
$$

when $0 \leq s \leq \delta$.

On the other hand 


$$
p(s), p(s)+i h \in D \quad 0<s .
$$

Thus, by (10), we have for $0<s \leq \delta$

$$
u^{\prime}(p(s)+i h)=u^{\prime}(p(s))
$$

from which it follows

$$
u^{\prime}(p+i h)=u^{\prime}(p) .
$$

Thus we have proved (10) provided only that $p, p+i h \in U_{1}(S)$ and $h \in H$. Therefore there exists a unique extension $u$ of $u^{\prime}$ to $U_{1}(S)$ $+i H=U_{1}(S)+H^{c}$ which is invariant by translation parallel to $i H$. Thus we conclude in view of (9) that $u$ is the desired function.

Q.E.D.

It is not known for us whether the function $u$ above is continuous or not. However, in $\S 5$ we shall prove using Lemma 2.2.10 the continuity of the restriction $\left.u\right|_{U(s)+3 H}$ provided that $H$ contains no semidefinite endomorphism except 0 .

\section{§3. Approximation Theorems}

3.1. Approximation Theorem on $U(n)$. In the preceding section $V$ was an $n$-dimensional Hilbert space, $\mathcal{G}$ the endomorphism ring of $V$. Let $\left\{e_{1}, e_{2}, \cdots, e_{n}\right\}$ be a fixed orthonormal base of $V$. Set, for $Z \in \mathcal{G}, Z$ $=\sum_{j, k} Z_{j k} e_{j} \otimes e_{k}^{*}$. Then the mapping $Z \rightarrow\left(Z_{j k}\right)$ gives an identification of $\mathcal{G}$ with the ring of complex $(n, n)$-matrices, which we shall preserve in whole discussion of this section. Let $\boldsymbol{G}$ denote the group of invertible elements of $\mathcal{G}$, i.e., the general linear group of order $n$, and $\boldsymbol{K}$ the unitary group of $V$. Thus $\boldsymbol{K}$ in the abbreviation of $U(n), \mathcal{G}$ is a Lie algebra by the usual cross-product and should be regarded as the Lie algebra of $\boldsymbol{G}$, and then the algebra of $\boldsymbol{K}$ is the Lie subalgebra of anti-hermitian endomorphisms of $\mathcal{G}$ denoted hereafter by $\mathcal{K}$.

Let $d Z$ denote the $\mathcal{G}$-valued 1 -form on $\boldsymbol{G}$ such that $d Z_{A}$ gives the usual identification of the tangent space at $A$ of $\mathcal{G}$ (or $\boldsymbol{G}$ ) with $\mathcal{G}$ for $A \in \boldsymbol{G}$, and let $Z$ denote the $\mathcal{G}$-valued function on $\boldsymbol{G}$ given by the inclu$\operatorname{sion} \boldsymbol{G} \rightarrow \mathcal{G}$. Then the $\mathcal{G}$-valued form $\delta Z=Z^{-1} d Z$ is the Maurer-Cartan 
form of $G$, that is

$$
\delta Z(\widetilde{A})=A \quad A \in \mathcal{G}
$$

where $\widetilde{A}$ denotes the left invariant vector field on $G$ which is the generator of the 1-parameter transformation $R_{\exp t A} . \quad\left(R_{z}\right.$ denotes the right multiplication by $Z$.) The $\delta Z$ is left invariant and it holds

$$
R_{A}^{*}(\delta Z)=\operatorname{ad}\left(A^{-1}\right) \delta Z \text {. }
$$

In terms of the coordinate system $\left(Z_{j k}\right)$ on $G, \delta Z$ can be written as follows:

$$
\delta Z=\left(\sum_{\mathfrak{l}}\left(Z^{-1}\right)_{j l} d Z_{l k}\right)
$$

where $\left(Z^{-1}\right)_{j k}, Z_{j k}$ must be considered as functions on $\boldsymbol{G}$.

The restriction $\iota^{*}(\delta Z)$ to $\boldsymbol{K}$ of $\delta Z$, where $c: \boldsymbol{K} \rightarrow \boldsymbol{G}$ is the inclusion map, is $\mathcal{K}$-valued and is the Maurer-Cartan form of $\boldsymbol{K}$. In the following, we often use $\omega$ instead of $\iota^{*}(\delta Z)$.

Take a non-zero element $v_{0}$ of $\bigwedge^{n 2} \mathcal{K}^{*}$ and define an $n^{2}$-form on $\boldsymbol{K}$

$$
v_{A}=\left(\Lambda^{t} \omega_{A}\right)\left(v_{0}\right) \quad A \in K
$$

where ${ }^{t} \omega_{A}$ denote the transposed of $\omega_{A}$. Then $v$ is left invariant, $v$ is also right invariant by (2) since

$$
D \mathcal{K}\left(\operatorname{ad}\left(A^{-1}\right)\right)=1 \quad A \in \boldsymbol{K}
$$

where $D \mathcal{K}(f)$ denotes the determinant of an endomorphism $f$ of $\mathcal{K}$. The formula (3) follows immediately from compactness of $\boldsymbol{K}$. Let [K] be the generator of $H_{n^{2}}(K, Z)$ such that $\int_{[K]} v>0$. Replacing $v_{0}$ by $c v_{0}(c>0)$ if necessary, we could choose $v_{0}$ so that

$$
\int_{[K]} v=1 \text {. }
$$

Let $\boldsymbol{T}$ denote the subgroup of $\boldsymbol{G}$ given by

$$
\boldsymbol{T}=\left\{A \in \boldsymbol{K}: A_{j k}=0 \text { for }(j, k) \text { such that } j \neq k\right\},
$$

and $\mathscr{I}$ the Lie algebra of $\boldsymbol{T}$, i.e.,

$$
\mathscr{I}=\left\{A \in \mathcal{K} ; A_{j k}=0 \text { for } j \neq k\right\} .
$$

Set also 


$$
\mathcal{K}_{1}=\left\{A \in \mathcal{K}: A_{11}=A_{22}=\cdots=A_{n n}=0\right\} .
$$

Then we have

$$
\begin{array}{lc}
\mathcal{K}=\mathcal{K}_{1} \oplus \mathscr{I} \quad \mathcal{K}^{*}=\mathcal{K}_{1}^{*} \oplus \mathcal{I}^{*} \\
\operatorname{ad}(T) \mathcal{K}_{1}=\mathcal{K}_{1} \quad T \in T \\
\operatorname{ad}(T) \mathscr{I}=\mathscr{I} \quad T \in T .
\end{array}
$$

As usual we shall make the canonical identification $\wedge\left(\mathcal{K}_{1}^{*} \oplus \mathscr{I}^{*}\right)$ $=\wedge \mathcal{K}_{1}^{*} \otimes \wedge \mathcal{I}^{*}$, the right hand side of which should be interpreted as the tensor product of the two graded algebras $\wedge \mathcal{K}_{1}^{*}$ and $\wedge \mathcal{I}^{*}$. Then

$$
\bigwedge^{n 2} \mathcal{K}^{*}=\left(\bigwedge^{n(n-1)} \mathcal{K}_{1}^{*}\right) \otimes\left(\bigwedge^{n} \mathscr{I}^{*}\right) \text {. }
$$

Now choose $v_{0}^{\prime} \in \bigwedge^{n(n-1)} \mathcal{K}_{1}^{*}$ and $v_{0}^{\prime \prime} \in \bigwedge^{n} \mathcal{I}^{*}$ so that

$$
v_{0}=v_{0}^{\prime} \otimes v_{0}^{\prime \prime}
$$

and define an $n$-form $v^{2}$ on $T$ and an $n(n-1)$-form $v^{\prime}$ on $K$

$$
\begin{aligned}
& v_{T}^{2}=\bigwedge^{t} \omega_{T}^{\prime}\left(v_{0}^{\prime \prime}\right) \quad T \in \boldsymbol{T} \\
& v_{A}^{\prime}=\bigwedge^{t} \omega_{A}\left(\bigwedge^{t} \pi_{1}\left(v_{0}^{\prime}\right)\right) \quad A \in \boldsymbol{K}
\end{aligned}
$$

where $\pi_{1}$ is the projection of $\mathcal{K}$ onto $\mathcal{K}_{1}$ in the splitting (5) and $\omega^{\prime}$ is the Maurer-Cartan form of $\boldsymbol{T}$ (i.e., the restriction to $\boldsymbol{T}$ of $\omega$ ). Then $v^{\prime}$ is left invariant and satisfies the following relations:

$$
\begin{aligned}
& R_{T}^{*}\left(v^{\prime}\right)=D \mathcal{K}_{1}\left(\operatorname{ad}\left(T^{-1}\right) \mid \mathcal{K}_{1}\right) v^{\prime}=v^{\prime} \\
& \widetilde{A}\rfloor v^{\prime}=0 \quad A \in \mathscr{I} .
\end{aligned}
$$

The last relation follows immediately from $A \sqcup \wedge^{t} \pi_{1}\left(v_{0}^{\prime}\right)=0$. Therefore there uniquely exists an $(n-1)$-form $v^{1}$ on $K / T$ such that

$$
v^{\prime}=\mathfrak{p}^{*}\left(v^{1}\right)
$$

where $\mathfrak{p}$ is the canonical projection $\boldsymbol{K} \rightarrow \boldsymbol{K} / \boldsymbol{T}$. We could choose $v_{0}^{\prime}$ so that $\int_{[K / T]} v^{1}=1$ for a suitable generator $[K / T]$ of $H_{n(n-1)}(K / T, Z)$. (Note that $K / T$ is orientable since $K / T$ is simply-connected.)

Now set $N^{\prime}=\boldsymbol{K} \times \boldsymbol{T}, N=\boldsymbol{K} / \boldsymbol{T} \times \boldsymbol{T}$. Then as made before we have the following canonical identifications: 


$$
\begin{aligned}
& \wedge T\left(N^{\prime}\right)=\wedge T(\boldsymbol{K}) \otimes \wedge T(\boldsymbol{T}) \\
& \wedge T(N)=\wedge T(\boldsymbol{K} / \boldsymbol{T}) \otimes \wedge T(\boldsymbol{T}) .
\end{aligned}
$$

Consider the map $\phi: N^{\prime} \rightarrow K$ defined by

$$
\phi(A, T)=A T A^{-1} .
$$

Since $\boldsymbol{T}$ is abelian, there exists a uniquely smooth mapping $\varphi: N \rightarrow \boldsymbol{K}$ for which the following diagram is commutative:

$$
\hat{\mathfrak{p}}=\mathfrak{p} \times i d_{\boldsymbol{T}} \underset{N \underset{\varphi}{\downarrow^{\prime}} \boldsymbol{N ^ { \prime }} \boldsymbol{K}}{\boldsymbol{\phi}}
$$

Then our key lemma is the following:

Lemma 3.1.1. $\varphi^{*}(v)=\sigma v^{1} \otimes v^{2}$ where $\sigma$ denotes the function on $\mathbf{T}$ defined by

$$
\sigma(T)=\prod_{j<k}\left|T_{j j}-T_{k k}\right|^{2}=\prod_{j<k}\left|1-T_{j j} T_{k k}\right|^{2} \quad \text { for } T \in \boldsymbol{T} .
$$

Proof. Since $v^{\prime} \otimes v^{2}=\mathfrak{p}^{*}\left(v^{1} \otimes v^{2}\right)$, it suffices to prove

$$
\phi^{*}(v)=\sigma v^{\prime} \otimes v^{2} .
$$

For $(A, T) \in N^{\prime}$ let $f_{(A, T)}$ be the linear mapping of $\mathcal{K} \oplus \mathscr{I}$ into $\mathcal{K}$ which makes the following diagram commutative:

$(*)_{1}$

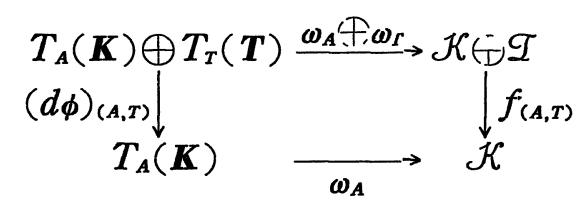

To determine $f_{(A, T)}$ we set

$$
A_{1}=A T A^{-1} \text {. }
$$

Then we have

$$
\begin{aligned}
d A_{1} & =(d A) T A^{-1}+A(d T) A^{-1}-A T A^{-1}(d A) A^{-1} \\
& =A T\left(A d\left(T^{-1}\right)-1\right) \delta A \cdot A^{-1}+A T \delta T A^{-1}
\end{aligned}
$$

where $\delta A=A^{-1} d A, \delta T=T^{-1} d T$. Thus we obtain

$$
\delta A_{1}=A_{1}^{-1} d A_{1}=a d(A)\left(\left(\operatorname{Ad}\left(T^{-1}\right)-1\right) \delta A+\delta T\right) .
$$


Therefore, for $X \in \mathcal{K}, Y \in \mathscr{I}$,

$$
\begin{aligned}
f_{(A, T)}(X, Y) & \left.=a d(A)\left(A d\left(T^{-1}\right)-1\right) X+Y\right) \\
& =a d(A) g_{T}\left(\pi_{1} \oplus i d_{\mathcal{Q}}\right)(X, Y)
\end{aligned}
$$

where $g_{T}$ denotes the endomorphism $\left.\left(A d\left(T^{-1}\right)-1\right)\right|_{\mathcal{K}_{1}} \oplus i d_{\mathscr{P}}$ of $\mathcal{K}(=$ $\left.\mathcal{K}_{1} \oplus \mathscr{I}\right)$. Hence

$$
\begin{aligned}
\wedge^{t} f_{(A, T)}\left(v_{0}\right) & =\Lambda^{t}\left(\pi_{1} \oplus i d_{\mathcal{Q}}\right)\left(\bigwedge^{t} g_{T}\left(v_{0}\right)\right) \\
& =D_{\mathcal{K}}\left(g_{T}\right)\left(\left(\bigwedge^{t} \pi_{1}\right) \otimes i d_{\bigwedge \mathscr{P}}\right)\left(v_{0}^{\prime} \otimes v_{0}^{\prime \prime}\right) \\
& =\sigma(T) \bigwedge^{t} \pi_{1}\left(v_{0}^{\prime}\right) \otimes v_{0}^{\prime \prime}
\end{aligned}
$$

since $D_{\mathcal{K}}\left(g_{T}\right)=\sigma(T)$. Consequently we deduce from (10), (11), (17) and diagram $(*)_{1}$,

$$
\phi^{*}(v)=\sigma v^{\prime} \otimes v^{2}
$$

Thus Lemma 3.1.1 is proved.

Now let $\boldsymbol{K}$, be the set of elements of $\boldsymbol{K}$ whose characteristic values are mutually distinct, then $\varphi^{-1}\left(\boldsymbol{K}_{r}\right)=\boldsymbol{K} / \boldsymbol{T} \times \boldsymbol{T}$, where $\boldsymbol{T}_{r}=\boldsymbol{K}_{r} \cap \boldsymbol{T}$. Obviously the measures of $\boldsymbol{K} \backslash \boldsymbol{K}_{r}, N \backslash \varphi^{-1}\left(\boldsymbol{K}_{r}\right)$ are zero. Therefore for a suitable generator $[N]$ of $H_{n^{2}}(N, Z)$ we have

$$
(n !)^{-1} \int_{[N]} \sigma v_{1} \otimes v^{2}=\int_{[K]} v
$$

since $\left.\varphi\right|_{\rho_{-1}\left(K_{r}\right)}$ is an $n$ !-hold covering. Thus applying Fubini's theorem, for the generator $[\boldsymbol{T}]$ of $H_{n}(\boldsymbol{T}, Z)$ such that $[\boldsymbol{K} / \boldsymbol{T}] \otimes[\boldsymbol{T}]=[N]$, we obtain

$$
\int_{[T]} \sigma v^{2}=n !
$$

However, for a suitable constant $c$,

$$
v^{2}=c \overline{t_{1} t_{2} \cdots t_{n}} d t_{1} \cdots d t_{n}
$$

where we denote by $t_{j}$ the function $T \rightarrow T_{j j}(T \in T)$. Consider the mapping $s$ from $\boldsymbol{R}^{n}$ onto $\boldsymbol{T}$ defined by

$$
s\left(\varphi_{1}, \cdots, \varphi_{n}\right)=\left(\begin{array}{lll}
e^{i \Phi_{1}} & & \\
& \ddots & \\
& & e^{i \varphi_{n}}
\end{array}\right) .
$$


Then

$$
s^{*}\left(\sigma v^{2}\right)=i^{n} c \underset{j<k}{\amalg}\left|e^{i \varphi_{j}}-e^{i \varphi_{k}}\right|^{2} d \varphi_{1} \wedge \cdots \wedge d \varphi_{n} .
$$

Simple computation shows

$$
\int_{0}^{2 \pi} \cdots \int_{0}^{2 \pi} \Pi_{j<k}\left|e^{i \varphi_{j}}-e^{i \varphi_{k}}\right| d \varphi_{1} \cdots d \varphi_{n}=(2 \pi)^{n} n ! .
$$

Therefore we have by (18) $c=(2 \pi i)^{-n}$, and hence

$$
v^{2}=(2 \pi i)^{-n} \overline{t_{1} \cdots t^{n}} d t_{1} \wedge \cdots \wedge d t_{n} .
$$

Now let $\tau_{\rho}(0<\rho<1)$ denote the diffeomorphism of $\boldsymbol{T}$ onto itself

$$
T \rightarrow\left(T-\rho I_{n}\right)\left(I_{n}-\rho T\right)^{-1}
$$

Then simple calculation shows

$$
\tau_{\rho}^{*}\left(\sigma v^{2}\right)=\left(\prod_{j=1}^{n} P\left(\rho, t_{j}\right)\right)^{n} \sigma v^{2}
$$

where

$$
P(\rho, t)=\left(1-\rho^{2}\right) /|1-\rho t|^{2} \text { for } t \text { such that }|t| \leq 1 \text {. }
$$

Set $\omega_{\rho}=\tau_{\rho}^{*}(\sigma v) / n$ !, then we have the following identities:

$$
\begin{aligned}
& \omega_{\rho}=\left(\prod_{j=1}^{n} P\left(\rho, t_{j}\right)\right)^{n} \omega_{0} \\
& \int_{[T]} \omega_{\rho}=1 \\
& n ! v^{1} \otimes \omega_{\rho}=\varphi^{*}\left(\mathfrak{p}_{\rho} v\right)
\end{aligned}
$$

where $\mathfrak{p}_{\rho}$ is the function on $\boldsymbol{K}$ given by

$$
\mathfrak{p}_{\rho}(A)=\left(1-\rho^{2}\right)^{n^{2}} /\left|D\left(I_{n}-\rho A\right)\right|^{2 n} \quad A \in \boldsymbol{K} .
$$

(21) follows from (18), and (23) follows from the fact that

$$
\left(\prod_{j=1}^{n} P\left(\rho, t_{j}\right)\right)^{n}=\varphi^{*}\left(\mathfrak{p}_{\rho}\right) \text {. }
$$

From (23) we can deduce

$$
\int_{[K]} \mathfrak{p}_{\rho} v=1
$$

Let us now proceed to prove an approximation theorem for continuous functions on $\boldsymbol{T}$. Define a neighbourhood of the identity $W_{\delta}$ $(0<\delta<2)$ setting 


$$
W_{\delta}=\left\{T \in T ; \operatorname{Re}\left(1-t_{j}\right)<\delta\right\} .
$$

Then the first approximation theorem is the following

Theorem 3.1.1. There exists a continuous function $c(\delta, \rho)>0$ $(0<\delta<2,0<\rho<1)$ such that

$$
\lim _{\rho \rightarrow 1} c(\delta, \rho)=0 \quad \text { for fixed } \delta .
$$

$$
\left|\int_{[T]} f \omega_{\rho}\right| \leq \sup _{T \in W_{\delta}}|f(T)|+c(\delta, \rho)\|f\|_{T} \quad f \in c(T)
$$

where $\|f\|_{T}=\sup _{T \in T}|f(T)|$.

Proof. Set

$$
\mathfrak{p}\left(\rho, \varphi_{1}, \cdots, \varphi_{m}\right)=\left(\prod_{j=1}^{m} P\left(\rho, e^{i \varphi_{j}}\right)\right)^{m} \prod_{1 \leq j<k \leq m}\left|e^{i \varphi_{j}}-e^{i \varphi_{k}}\right|^{2} .
$$

Then by (21) we have

$$
\int_{-\pi}^{\pi} \cdots \int_{-\pi}^{\pi} \mathfrak{p}\left(\rho, \varphi_{1}, \cdots, \varphi_{m}\right) d \varphi_{1} \cdots d \varphi_{m}=(2 \pi)^{m} m !
$$

and

$$
(2 \pi)^{n} n !\left|\int f \omega_{\rho}\right|=\left|\int_{-\pi}^{\pi} \cdots \int_{-\pi}^{\pi} g\left(\varphi_{1}, \cdots, \varphi_{n}\right) \mathfrak{p}\left(\rho, \varphi_{1}, \cdots, \varphi_{n}\right) d \varphi_{1} \cdots d \varphi_{n}\right|
$$

where $g=s^{*}(f)$.

If $1-\cos \varphi_{j}<\delta$ for $j=1,2, \cdots, n$, then

$$
\left|g\left(\varphi_{1}, \cdots, \varphi_{n}\right)\right| \mathfrak{p}\left(\rho, \varphi_{1}, \cdots, \varphi_{n}\right) \leq \sup _{T \in W_{\delta}}|f(T)| \mathfrak{p}\left(\rho, \varphi_{1}, \cdots, \varphi_{n}\right) .
$$

If $1-\cos \varphi_{1}>\delta$, then

$$
\left|g\left(\varphi_{1}, \cdots, \varphi_{n}\right)\right| \mathfrak{p}\left(\rho, \varphi_{1}, \cdots, \varphi_{n}\right) \leq C(1-\rho)(\rho \delta)^{-n}\left\|f^{\prime}\right\|_{T} \mathfrak{p}\left(\rho, \varphi_{2}, \cdots, \varphi_{n}\right)
$$

for some constant $C>0$, for we have then

$$
\begin{aligned}
\mathfrak{p}\left(\rho, \varphi_{1}, \cdots, \varphi_{n}\right) / \mathfrak{p}\left(\rho, \varphi_{2}, \cdots, \varphi_{n}\right) \\
\quad=P\left(\rho, e^{i \Phi_{1}}\right)^{n} \prod_{1<j} P\left(\rho, e^{i \Phi_{j}}\right) \prod_{1<k}\left|e^{i \Phi_{1}}-e^{i \Phi_{k}}\right|^{2} \\
\quad \leq\left(\frac{1-\rho}{2 \delta \rho}\right)^{n}\left(\frac{2}{1-\rho}\right)^{n-1} \cdot 2^{2(n-1)}=C(1-\rho) /(\rho \delta)^{n}
\end{aligned}
$$

using the following inequalities: 


$$
\begin{aligned}
& p\left(\rho, e^{i \varphi_{j}}\right)=\frac{1-\rho^{2}}{(1-\rho)^{2}+2 \rho\left(1-\cos \varphi_{j}\right)} \leq \frac{1-\rho^{2}}{(1-\rho)^{2}} \leq \frac{2}{1-\rho} \\
& p\left(\rho, e^{i \varphi_{1}}\right)=\frac{1-\rho^{2}}{(1-\rho)^{2}+2 \rho\left(1-\cos \varphi_{1}\right)} \leq \frac{1-\rho^{2}}{2 \rho\left(1-\cos \varphi_{1}\right)} \leq \frac{1-\rho}{\rho \delta} .
\end{aligned}
$$

Thus we obtain, if one sets $1-\cos \delta^{\prime}=\delta 0<\delta^{\prime}<\pi$,

$$
\begin{aligned}
& (2 \pi)^{n} n !\left|\int f \omega_{\rho}\right| \\
& \leq \int_{\left|\Phi_{1}\right|<\delta^{\prime}} \cdots \int_{\left|\Phi_{n}\right|<\delta^{\prime}} g\left(\varphi_{1}, \cdots, \varphi_{n}\right) \mid \mathfrak{p}\left(\rho, \varphi_{1}, \cdots, \varphi_{n}\right) d \varphi_{1} \cdots d \varphi_{n} \\
& \quad+\int_{1-\cos \Phi_{1} \leq \delta} d \varphi_{1} \int_{-\pi}^{\pi} \cdots \int_{-\pi}^{\pi}\left|g\left(\varphi_{1}, \cdots, \varphi_{n}\right)\right| \mathfrak{p}\left(\rho, \varphi_{1}, \cdots, \varphi_{n}\right) d \varphi_{2} \cdots d \varphi_{n} \\
& \quad+\cdots+\int_{1-\cos \Phi_{n} \leq \delta} d \varphi_{n} \int_{-\pi}^{\pi} \cdots \int_{-\pi}^{\pi}\left|g\left(\varphi, \cdots, \varphi_{n}\right)\right| \mathfrak{p}\left(\rho, \varphi_{1}, \cdots, \varphi_{n}\right) d \varphi_{1} \cdots d \varphi_{n-1} \\
& \leq(2 \pi)_{n} n ! \sup _{T \in W_{\delta}}|f(T)|+(2 \pi)^{n} n ! C(1-\rho)(\rho \delta)^{-n}\|f\|_{T}
\end{aligned}
$$

where we used (27), (28), (29) in the last step. Thus

$$
\left|\int f \omega_{\rho}\right| \leq \sup _{T \in W_{\delta}}|f(T)|+c(\rho, \delta)\|f\|_{\boldsymbol{T}}
$$

where $c(\rho, \delta)=\frac{C(1-\rho)}{\rho^{n} \delta^{n}}$.

Q.E.D.

Now we shall prove an approximation theorem for continuous functions on $\boldsymbol{K}$. First we prepare some notations. Given a continuous function $f$ on $\boldsymbol{K}$, let $f_{\rho}(0<\rho<1)$ denote the continuous function $f_{\rho}$ on $\boldsymbol{K}$ given by

$$
f_{\rho}(A)=\int_{[\boldsymbol{K}]} L_{A}^{*}(f) \mathfrak{p}_{\rho} v \quad A \in \boldsymbol{K}
$$

where $L_{A}$ denotes the left multiplication by $A$. In view of the invariance of $v, f_{\rho}$ can be also given by

$$
f_{\rho}(A)=\int_{[K]} f L_{A^{-1}}^{*}\left(\mathfrak{p}_{\rho}\right) v \quad A \in \boldsymbol{K} .
$$

From (30) we have

$$
L_{A}^{*}\left(f_{\rho}\right)=\left(L_{A}^{*}(f)\right)_{\rho} \quad A \in \boldsymbol{K}
$$

and from (31) and the fact that $L_{B}^{*}\left(\mathfrak{p}_{\rho}\right)=R_{B}^{*}\left(\mathfrak{p}_{\rho}\right) B \in K$ it follows 


$$
R_{A}^{*}\left(f_{\rho}\right)=\left(R_{A}^{*}(f)\right)_{\rho} \quad A \in \boldsymbol{K} .
$$

(31) shows also the real analyticity of $f_{\rho}$ since $L_{A}^{*-1}\left(\mathfrak{p}_{\rho}\right)$ is real analytic with respect to $A$. Now our second approximation theorem is the following

Theorem 3.1.2. $\left\|f_{\rho}-f\right\|_{K}=\sup _{A \in K}\left|f_{\rho}(A)-f(A)\right| \rightarrow 0$ as $\rho \rightarrow 1$.

Proof. Denote by $V_{\delta}$ the neighbourhood of the identity in $\boldsymbol{K}$ given by

$V_{\delta}=\left\{A \in K: \operatorname{Re}(1-\xi)<\delta\right.$ for any $\xi$ such that $\left.D\left(\xi I_{n}-A\right)=0\right\}$, that is, $V_{\delta}=\varphi\left(\boldsymbol{K} / \boldsymbol{T} \times W_{\delta}\right) \quad\left(\right.$ or $\left.\boldsymbol{K} / \boldsymbol{T} \times W_{\delta}=\varphi^{-1}\left(V_{\delta}\right)\right)$. Then $\left\{V_{\delta}\right\}_{0<\delta<2}$ forms a complete system of neighbourhoods at the identity of $\boldsymbol{K}$. Since $\boldsymbol{K}$ is a compact group, $f$ is equicontinuous on $\boldsymbol{K}$, i.e., for any $\varepsilon>0$ there exists $\delta(0<\delta<2)$ such that

$$
\sup _{A \in V_{\delta}}\left|L_{A}^{*}(f)(B)-f(A)\right| \leq \varepsilon .
$$

On the other hand we have by (24)

$$
f_{\rho}(A)-f(A)=\int_{K}\left(L_{A}^{*}(f)-f(A)\right) \mathfrak{p}_{\rho} v .
$$

Therefore, if one sets $g_{A}=\varphi^{*}\left(L_{A}^{*}(f)-f(A)\right)$, then by (34)

$$
\sup _{(m, T) \in \mathbf{K} / T \times W_{\delta}}\left|g_{A}(m, T)\right| \leq \varepsilon,
$$

in particular

$$
\sup _{T \in W_{\delta}}\left|\hat{g}_{A}(T)\right| \leq \varepsilon
$$

where we have set

$$
\hat{g}_{A}(T)=\int_{[K / T]} g_{A}(m, T) v^{1} .
$$

Using Fubini's theorem we deduce from (23), (35)

$$
\begin{aligned}
f_{\rho}(A)-f(A) & =\int_{[N]} g_{A} v^{1} \otimes \omega_{\rho} \\
& =\int_{[T]} \hat{g}_{A} \omega_{\rho} .
\end{aligned}
$$

Therefore by Theorem 3.1.1 and (36) 


$$
\begin{aligned}
\left|f_{\rho}(A)-f(A)\right| & \leq_{\varepsilon}+c(\rho, \delta)\left\|\hat{g}_{A}\right\|_{T} \\
& \leq_{\varepsilon}+2 c(\rho, \delta)\|f\|_{K} .
\end{aligned}
$$

Since the right hand side is independent of $A$, we obtain

$$
\left\|f_{\rho}-f\right\|_{K} \leq \varepsilon+2 c(\rho, \delta)\|f\|_{K} .
$$

Letting $\rho$ tend to 1 from below,

$$
\varlimsup_{\rho \rightarrow 1}\left\|f_{\rho}-f\right\|_{K} \leq \varepsilon .
$$

Since $\varepsilon$ is arbitrary, we finally obtain

$$
\varlimsup_{\rho \rightarrow 1}\left\|f_{\rho}-f\right\|_{K}=0 \text {. }
$$

Q.E.D.

Corollary 3.1.1. For $f \in C(\boldsymbol{K})$ there exists a holomorphic function $f_{\rho}^{*}$ on $\boldsymbol{G}_{\rho}$ such that $\left.f_{\rho}^{*}\right|_{\boldsymbol{K}}=f_{\rho}$, where $\boldsymbol{G}_{\rho}$ denotes the open set

$$
\left\{Z \in G ; \rho^{-2} I_{n}>Z Z^{*}>\rho^{2} I_{n}\right\} .
$$

To prove this we assume the following lemma.

Lemma $\boldsymbol{H}_{1}$ (Hua [4]). For $Z \in G_{\rho}$ and for $A \in \boldsymbol{K}$, the matrices

$$
I_{n}-\rho Z^{-1} A, \quad I_{n}-\rho A^{-1} Z
$$

are non-singular.

Proof of Corollary 3.1.1. In view of this lemma define $\mathfrak{p}_{\rho}^{z} \in C(\boldsymbol{K})$, setting

$$
\mathfrak{p}_{\rho}^{Z}(A)=\left(1-\rho^{2}\right)^{n^{2}} /\left(D\left(I_{u}-\rho Z^{-1} A\right) D\left(I_{n}-\rho A^{-1} Z\right)\right)^{n} \quad Z \in G_{\rho} .
$$

Note that $\mathfrak{p}_{\rho}^{A}=L_{-A}^{*}\left(\mathfrak{p}_{\rho}\right)$ for $A \in \boldsymbol{K}$. Thus, if one sets for $Z \in G_{\rho}$

$$
f_{\rho}^{*}(Z)=\int f \mathfrak{p}_{\rho}^{z} v
$$

it holds $\left.f_{\rho}^{*}\right|_{\kappa}=f_{\rho}$ and $f_{\rho}^{*}$ is holomorphic since $\mathfrak{p}_{\rho}^{Z}(A)$ is holomorphic with respect to $Z$ in $\boldsymbol{G}_{\rho}$.

Q.E.D.

3. 2. Application to Some Type of Vector Fields on Trivial $U(n)$ BundLE. Here we shall study how the map $f \rightarrow f_{p}$ (resp $f \rightarrow f_{p}^{*}$ ) relates left $\boldsymbol{K}$-invariant vector fields on the product of $\boldsymbol{K}$ (resp $\boldsymbol{G}_{p}$ ) with an open subset in $\boldsymbol{R}^{N}$. 
Let $\mathfrak{p}_{\rho}^{*}$ denote the function on $\boldsymbol{K} \times \boldsymbol{G}_{\rho}$

$$
(A, Z) \rightarrow \mathfrak{p}_{\rho}^{Z}(A) \text {. }
$$

Then $\mathfrak{p}_{\rho}^{*}$ satisfies the following relation

$$
\mathfrak{p}_{\rho}^{*}(A B, Z)=\mathfrak{p}_{\rho}^{*}\left(A, Z B^{-1}\right)=\mathfrak{p}_{\rho}^{*}\left(B, A^{-1} Z\right) .
$$

Choose a $\boldsymbol{K}$-invariant volume $v^{*}$ on $\boldsymbol{G}$ (which certainly exists since $\boldsymbol{K}$ is compact) and for $\psi \in C_{0}^{\infty}\left(\boldsymbol{G}_{\rho}\right)$ define a function on $\boldsymbol{K}$ setting

$$
\psi(\rho)=\int_{G} \psi_{(\rho)}^{A} v^{*} \quad A \in K
$$

where $\mathfrak{p}_{(\rho)}^{A}$ is the function on $\boldsymbol{G}_{\rho}$ defined by $\mathfrak{p}_{(\rho)}^{A}(Z)=\mathfrak{p}_{\rho}^{*}(A, Z)$. Here, for definiteness, the orientation of $\boldsymbol{G}$ in the integration should be the natural orientation of $\boldsymbol{G}$ as a complex manifold. From the invariance of $v^{*}$ and from (39) it follows that

$$
\begin{aligned}
& L_{A}(\psi(\rho))=\left(L_{A} \psi\right)_{(\rho)} \\
& R_{A}(\psi(\rho))=\left(R_{A} \psi\right)_{(\rho)}
\end{aligned}
$$

where $A \in \boldsymbol{K}$. From (33), (41) we obtain

Proposition 3. 2. 1. For $A \in \boldsymbol{K}$

$$
\begin{aligned}
& \widetilde{A}(\psi(\rho))=(\widetilde{A} \psi)_{(\rho)} \quad \psi \in C_{0}^{\infty}\left(G_{\rho}\right) \\
& \widetilde{A}\left(f_{\rho}\right)=(\widetilde{A} f)_{\rho} \quad f \in C^{\infty}(\boldsymbol{K}) .
\end{aligned}
$$

In (43) $\widetilde{A}$ in the left hand side should be the left invariant vector field corresponding to $A$ on $\boldsymbol{K}$ and $\widetilde{A}$ in the right should be that on $\boldsymbol{G}$.

Later we also need

Proposition 3.2.2. For $A \in K$ and for $\varphi, \psi \in C_{0}^{\infty}(G)$

$$
\int_{G}(\widetilde{A} \varphi) \psi v^{*}+\int_{G} \varphi(\widetilde{A} \psi) v^{*}=0
$$

Proof. Note that

$$
\int_{G}((\widetilde{A} \varphi) \psi+\varphi(\widetilde{A} \psi)) v^{*}=\int_{G} \widetilde{A}(\varphi \psi) v^{*}=\left[\frac{d}{d t} \int_{G} R_{\exp t A}^{*}(\varphi \psi) v^{*}\right]_{t=0} .
$$

But $\int_{G} R_{\exp t A}^{*}(\psi) v^{*}$ is independent of $t$ because of the $\boldsymbol{K}$-invariance of 
$v^{*}$, therefore (44) is proved.

In the same way we obtain

Proposition 3.2.2'. For $A \in \boldsymbol{K}$ and for $f, g \in C_{0}^{\infty}(\boldsymbol{K})$

$$
\int_{K}(\widetilde{A} f) g v+\int_{K} f(\widetilde{A} g) v=0
$$

Now let $\Omega$ be a domain in $\boldsymbol{R}^{N}$ and denote by $M, \widetilde{M}$ the product manifolds $K \times \Omega, G \times \Omega$, respectively, then $T_{(A, x)}(M), T_{(z, x)}(\widetilde{M})$ can be canonically identified with $T_{A}(\boldsymbol{K}) \oplus \boldsymbol{T}_{x}(\Omega), T_{z}(\boldsymbol{G}) \oplus T_{x}(\Omega)$ respectively and under this identification we call a vector field on $M(\operatorname{resp} \widetilde{M}$ ) a vertical vector field if its value at each point $(A, x)$ of $M(\operatorname{resp}(Z, x)$ of $\tilde{M}$ ) lies in $T_{A}(\boldsymbol{K})$ (resp $T_{Z}(\boldsymbol{G})$ ), further a vector field on $M$ (resp $\widetilde{M})$ is called a horizontal vector field if its value at each point $(A, x)$ of $M(\operatorname{resp}(Z, x)$ of $\tilde{M})$ lies in $T_{x}(\Omega)$. Then every vector field $X$ on $M$ (or on $\widetilde{M}$ ) can be uniquely written as the sum of a vertical vector field and a horizontal vector field. The former is called the vertical part of $X$ and denoted by $X^{v}$ and the latter is called the horizontal part of $X$ and denoted by $X^{h}$. A vector field $X$ on $M$ $(\operatorname{resp} \widetilde{M})$ is called left invariant if, for any $A$ of $\boldsymbol{K}$ (resp $Z$ of $\boldsymbol{G}$ ),

$$
d L_{A}(X)=X \quad\left(\text { resp. } d L_{z}(X)=X\right)
$$

where $L_{A}, L_{z}$ are transformations given by

$$
\begin{array}{ll}
L_{A}\left(A^{\prime}, x\right)=\left(A A^{\prime}, x\right) & \left(A^{\prime}, x\right) \in M \\
L_{z}\left(Z^{\prime}, x\right)=\left(Z Z^{\prime}, x\right) & \left(Z^{\prime}, x\right) \in \widetilde{M}
\end{array}
$$

The horizontal part and the vertical part of a left invariant vector field $X$ on $M$ (resp. on $\widetilde{M}$ ) can be written as follows:

$$
\begin{aligned}
& X^{k}=\sum_{i=1}^{N} c_{i}(x) \frac{\partial}{\partial x_{i}} \\
& X^{v}=\widetilde{A(x)} \quad(\operatorname{resp} \widetilde{Z(x))}
\end{aligned}
$$

where $\left(x_{1}, \cdots, x_{N}\right)$ is the system of coordinates of $\boldsymbol{R}^{N}$ and $c_{i}(x)$ are $C^{\infty}$ functions on $\Omega$, and $A(x)(\operatorname{resp} Z(x))$ is a $\mathcal{K}$ (resp $\mathcal{G}$ )-valued function on $\Omega$. Here $\widetilde{A(x)}$ means the vector field which assigns to each point 


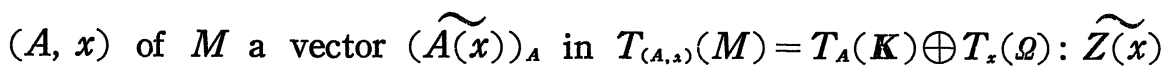
should be interpreted in the same way. Note that also $A(x)$ can be regarded as a $\mathcal{G}$-valued function, therefore for a left invariant vector field $X$ on $M$, there uniquely exists a left invariant vector field $\widehat{X}$ on $\widetilde{M}$ such that

$$
\widehat{X}_{p}=X_{p} \text { for } p \in M \text {. }
$$

We call this $\hat{X}$ the extension of $X$. A left invariant vector field is called typical if $X^{h}$ has constant coefficients, i.e., it can be written as follows:

$$
X^{h}=\sum_{i=1}^{N} c_{i} \frac{\partial}{\partial x_{i}} \quad\left(c_{1}, \cdots, c_{N} \text { are constants }\right) .
$$

Now let $\boldsymbol{v}, \boldsymbol{v}^{*}$ be volumes on $M, \widetilde{M}$ defined by

$$
v=d x \otimes v, \quad \boldsymbol{v}^{*}=d x \otimes v^{*}
$$

where $d x=d x^{\mathrm{i}} \cdots e x_{N}$. Then we have

Proposition 3.2.3. The formal adjoint with respect to volume $\boldsymbol{v}\left(\operatorname{resp} v^{*}\right)$ of a typical vector field $X$ on $M(\operatorname{resp} \hat{X}$ on $\widetilde{M})$ is $-X$ $($ resp $-\widehat{X})$, i.e.,

$$
\begin{aligned}
& \int_{M}(X f) g v+\int_{M} f(X g) v=0 \quad f, g \in C_{0}^{\infty}(M) \\
& \left(\operatorname{resp} \int_{\widetilde{M}}(\widehat{X} \varphi) \psi v^{*}+\int_{\widetilde{M}} \varphi\left(\widehat{X}_{\varphi}\right) v^{*}=0 \quad \varphi, \psi \in C_{0}^{\infty}(\widetilde{M})\right) .
\end{aligned}
$$

Proof. (45) for the horizontal parts of $X, \widehat{X}$ is obvious, and for the vertical parts it is an immediate consequence of Propositions 3.2.1, 3.2. 2 and Fubini's theorem.

Now we shall extend mappings $f \rightarrow f_{\rho}, f \rightarrow f_{\rho}^{*}$ for $f \in C(\boldsymbol{K})$ and mapping $\psi \rightarrow \psi(\rho)$ for $\psi \in C_{0}^{\infty}\left(\boldsymbol{G}_{\rho}\right)$ into mappings of $C(M)$ and of $C_{0}^{\infty}\left(\widetilde{M}_{\rho}\right)$ where $\widetilde{M}_{\rho}=G_{\rho} \times \Omega$. For $f \in C(M)$ and $x \in \Omega$ let $f^{x}$ denote the function on $\boldsymbol{K}$ given by $f^{x}(A)=f(A, x)(A \in \boldsymbol{K})$, and define $f_{\mathrm{p}}$ by

$$
f_{\rho}(A, x)=\left(f^{x}\right)_{\rho}(A)
$$

and $f_{\rho}^{*}$ setting

$$
f_{\rho}^{*}(Z, x)=\left(f^{*}\right)_{\rho}^{*}(Z) \quad Z \in \boldsymbol{G}_{\rho} .
$$


In the same way we shall define $\psi(\rho)$ for $\psi \in C_{0}^{\infty}\left(\widetilde{M}_{\rho}\right)$ :

$$
\psi_{(\rho)}(A, x)=\left(\psi^{x}\right)_{(\rho)}(A) \quad A \in K, x \in \Omega
$$

where $\psi^{x}$ denotes the function on $G_{\rho}$ given by $\psi^{x}(Z)=\psi(Z, x)$. Then we obtain an analogy of Theorem 3.1.2.

Theorem 3.1.2'. For $f \in C(M), f_{\rho}$ tends to $f$ in the topology of $C(M)$ as $\rho / 1$.

Proof. Let $K$ be a compact subset of $\Omega$, then the set $\left\{f^{x}: x \in K\right\}$ is compact in $C(\boldsymbol{K})$ since the map $x \rightarrow f_{x}$ is continuous. But the convergence $g_{\rho} \rightarrow g$ in $C(\boldsymbol{K})$ is uniform on any compact subset of $C(\boldsymbol{K})$ since by definition

Thus

$$
\left\|g_{\rho}\right\|_{\boldsymbol{K}} \leq\|g\|_{\boldsymbol{K}} \quad g \in C(\boldsymbol{K}) .
$$

$$
\sup _{(A, x) \in K \times K}\left|f_{\rho}(A, x)-f(A, x)\right| \rightarrow 0 \text { as } \rho \nearrow 1 \text {. }
$$

Q.E.D.

Now (43), (43)' are generalized as follows.

Proposition 3.2.4. If $X$ is a typical vector field on $M$, then

$$
\begin{array}{ll}
(X f)_{p}=X\left(f_{\rho}\right) & f \in C^{\infty}(M) \\
(\widehat{X} \Psi)_{(\rho)}=X\left(\psi_{(\rho)}\right) & \psi \in C_{0}^{\infty}\left(\widetilde{M}_{\rho}\right) .
\end{array}
$$

These formulae are evident by Proposition 3.2.1 and the structure of typical vector fields on $M$.

Theorem 3.2.1. Let $X$ be a typical vector field on $M$ and $f$ be a continuous function on $M$ such that $X f=0$ in the distribution sense. Then we have $X f_{p}=0, \widehat{X} f_{\rho}^{*}=0$.

Proof. Continuous mappings $f \rightarrow X\left(f_{\rho}\right), f \rightarrow(X f)_{\rho}$ of $C(M)$ into $\mathscr{D}^{\prime}(M)$ coincide on dense subset $C^{\infty}(M)$ of $C(M)$, therefore there coincide on the whole space $C(M) . X\left(f_{\rho}\right)=0$ is proved. In view of Proposition 3.2.2 $\widehat{X}\left(f_{\rho}^{*}\right)=0$ is equivalent to $\int_{\widetilde{M}} f_{\rho}^{*}(\widehat{X} \Psi) v^{*}=0\left(\forall \psi \in C_{0}^{\infty}\left(\widetilde{M}_{\rho}\right)\right)$. But by Fubini's theorem

$$
\int_{\widetilde{M}} f_{\rho}^{*}(\widehat{X} \psi) v^{*}=\int_{M} f(\widehat{X} \psi)_{(\rho)} v=\int_{M} f X(\psi(\rho)) v
$$


where in the last step we have used Proposition 3.2.4. Since $\psi_{(\rho)}$ has compact support, we have by the hypothesis

$$
\int_{\widetilde{M}} f_{p}^{*}(\widehat{X} \psi) \boldsymbol{v}^{*}=0
$$

Q.E.D.

Remark. Evidently Propositions 3.2.3 and 3.2.4 and Theorem 3.2.1 are still valid for a differential operator $X+i X^{\prime}$, where $X, X^{\prime}$ are typical vector fields. Such an operator will be called also a typical vector field.

To end this section we add an interesting approximation theorem for solutions of certain overdetermined system of differential equations, but we do not use this in later discussion.

Theorem 3.2.2. Let $X_{1}, \cdots, X_{N}$ be real analytic typical vector fields such that the differential operator with constant coefficients $X_{1}^{h}, \cdots, X_{N}^{h}$ are linearly independent. Then the set of real analytic solutions of the system of equations

$$
X_{1} f=0, \cdots, X_{N} f=0
$$

is dense in the space of all continuous solutions of it with respect to the relative topology induced from $C(M)$.

Proof. In view of Theorem 3.1. $1^{\prime}$ it suffices to prove that $f_{\rho}$ is real analytic for a solution $\hat{f}$, which follows immediately from the real analyticity of $f_{\rho}^{*}$. By Theorem 3.2.1 we have

$$
\widehat{X}_{1} f_{\rho}^{*}=0, \cdots, \widehat{X}_{N} f_{\rho}^{*}=0 .
$$

Moreover since $f_{\rho}^{*}(Z, x)$ is holomorphic in variables $Z_{j k}(1 \leq j, k \leq n)$, we have

$$
\frac{\partial}{\partial \bar{Z}_{j k}} f_{\rho}^{*}=0 \quad(1 \leq j, k \leq n) .
$$

Since the system of differential equation (48), (49) is elliptic by the assumption of the theorem, $f_{p}^{*}$ is real analytic.

Q.E.D. 


\section{§4. Problem $\boldsymbol{H}$ for $\boldsymbol{D}_{0}$}

4.1. A Local Parametrization of $D_{0}$. In this section we investigate Problem $H$ for the Šilov boundary $D_{0}$ of the Siegel domain of second kind $D$ in Section 2. For this purpose we shall give a more convenient parametrization of $D_{0}$ in order to apply the results of the preceding section to $D_{0}$.

Recall that $D_{0}$ was given by

$$
D_{0}=\left\{(Z, x) \in \mathcal{G} \times V ; \frac{1}{i}\left(Z-Z^{*}\right)-x \otimes x^{*}>0\right\}
$$

where $V$ is an $n$-dimensional complex Hilbert space and $\mathcal{G}$ the endomorphism ring of $V$. Fixing an orthonormal base $\left\{e_{1}, \cdots, e_{n}\right\}$ of $V$ as before, we shall identify $V$ with $\boldsymbol{C}^{n}, \mathcal{G}$ with $M_{n}(\boldsymbol{C})$, where $\boldsymbol{C}^{n}$ should be regarded as the space of column vectors with $n$ components. Let $\boldsymbol{H}$ denote the subgroup of $\boldsymbol{G}$ given by

$$
\boldsymbol{H}=\left\{\left(\begin{array}{lll}
h_{1} & & 0 \\
& \ddots & \\
0 & & h_{n}
\end{array}\right) ; h_{1}, \cdots, h_{n} \in C \backslash\{0\}\right\}
$$

and $\mathfrak{h}$ the Lie algebra of $\boldsymbol{H}$, i.e.,

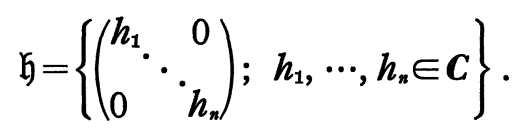

We set further

$$
\mathfrak{h}_{r}=\left\{\left(\begin{array}{lll}
h_{1} & & 0 \\
& \ddots & \\
0 & & h_{n}
\end{array}\right) \in \mathfrak{h} ; \quad h_{j} \neq h_{k} \text { if } j \neq k\right\} .
$$

Now define a mapping $\bar{\phi}$ of $\boldsymbol{G} \times \mathfrak{h}_{r}$ into $\mathcal{G} \times V$ by setting

$$
\varphi(Z, H)=\left(Z H Z^{-1}, Z \cdot x_{0}\right) ; \quad Z \in G, H \in \mathfrak{h}_{r}
$$

where $x_{0}=\left(\begin{array}{c}1 \\ \cdots \\ 1\end{array}\right)$. Then by simple calculation one can easily see that $(d \varphi)_{p}$ is non-singular at each point $p$ of $\boldsymbol{G} \times \mathfrak{h}_{r}$. Let us determine $\bar{\phi}\left(\boldsymbol{G} \times \mathfrak{h}_{r}\right)$. For this purpose let $\mathcal{G}_{r}$ be the set of elements in $\mathcal{G}$ whose characteristic polynomial has mutually distinct roots, i.e., $\mathcal{G}=\{Z \in \mathcal{G}$ : the discriminant of equation in $\lambda D\left(\lambda I_{n}-Z\right)=0$ is non-zero $\}$ and for any 
permutation $\sigma$ of $n$ letters $\{1,2, \cdots, n\}$ define $(\sigma) \in G$ as follows:

$$
(\sigma)_{j k}= \begin{cases}1 & \text { if } \sigma(k)=j \\ 0 & \text { if } \sigma(k) \neq j .\end{cases}
$$

Further we shall denote by $W$ the set of all ( $\sigma)$. Then $W$ is a finite subgroup of $\boldsymbol{G}$ and we have

$$
\text { (б) } \boldsymbol{H}(\sigma)^{-1}=\boldsymbol{H}
$$

Therefore $\boldsymbol{N}=\boldsymbol{W} \boldsymbol{H}=\boldsymbol{H} W$ is a subgroup of $\boldsymbol{G}$. It is well-known that $\boldsymbol{N}$ is the normalizer of $\boldsymbol{H}$ in $\boldsymbol{G}$. Obviously

$$
\boldsymbol{N} / \boldsymbol{H} \cong \boldsymbol{W}
$$

Suppose that $\zeta \in \mathcal{G}_{r}$, then, by Linear Algebra, there exists an element $Z$ of $\boldsymbol{G}$ and an element $H$ of $\mathfrak{h}_{r}$ such that

$$
Z H Z^{-1}=\zeta \text {. }
$$

Define a mapping $S: \mathcal{G}_{r} \rightarrow \boldsymbol{G} / \mathbf{N}$ setting

$$
S(\zeta)=\pi(Z)
$$

where $\pi$ is the canonical projection of $G$ onto $G / N$. The $S(\zeta)$ is independent of the choise of such $Z$, and $S$ is well-defined. It is also evident that $S$ is a holomorphic mapping. Define a function $f_{0}$ on $V$ setting

$$
f_{0}(x)=x_{1}^{2} \cdots x_{n}^{2} \quad x=\left(\begin{array}{c}
x_{1} \\
\vdots \\
x_{n}
\end{array}\right)
$$

and set for $(Z, x) \in G \times V$

$$
f_{1}(Z, x)=f_{0}\left(Z^{-1} x\right) .
$$

Then $f_{1}(Z N, x)=(D(N))^{-2} f_{1}(Z, x)$ for $N \in N$. Therefore, if one sets

$$
f_{2}(Z, x)=D(Z)^{2} f_{1}(Z, x),
$$

we have $f_{2}(Z N, x)=f_{2}(Z, x)$. Hence there exists a function $f_{3}$ defined on $\boldsymbol{G} / \boldsymbol{N} \times V$ such that

$$
f_{3}(\pi(Z), x)=f_{2}(Z, x) .
$$


Set $f(\zeta, x)=f_{3}(S(\zeta), x)$ for $(\zeta \times x) \in \mathcal{G}_{r} \times V$. Then $f$ is a holomorphic function on $\mathcal{G}_{r} \times V$.

Let $R$ be the subset of $\mathcal{G}_{r} \times V$ defined as follows

$$
R=\left\{(\zeta, x) \in \mathcal{G}_{r} \times V ; f(\zeta, x) \neq 0\right\} .
$$

Since the complement of $\mathcal{G}_{r} \times V$ is an analytic variety and since $f$ is holomprphic, $D_{r}=R \cap D_{0}$ is open, dense in generic submanifold $D_{0}$. We claim that

$$
R=\varphi\left(\boldsymbol{G} \times \mathfrak{h}_{r}\right) .
$$

$R \supseteq \varphi\left(\boldsymbol{G} \times \mathfrak{h}_{r}\right)$ is obvious, in fact $Z H Z^{-1} \in \mathcal{G}^{r}, f\left(Z H Z^{-1}, Z x_{0}=D(Z)^{2}\right.$ $\times f_{0}\left(x_{0}\right) \neq 0$ for $(Z, H) \in \boldsymbol{G} \times \mathfrak{h}_{r}$. To show $R \subseteq \varphi\left(\boldsymbol{G} \times \mathfrak{h}_{r}\right)$ note that for any $(\zeta, x) \in R$ there exists $(Z, H) \in G \times \mathfrak{h}$, such that

(1) $Z H Z^{-1}=\zeta$.

Then we have

$$
f_{0}\left(Z^{-1} x\right) \neq 0
$$

since $f(\zeta, x)=f_{3}(\pi(Z), x)=f_{2}(Z, x)=f_{0}\left(Z^{-1} x\right) D(Z)^{2}$. By the definition of $f_{0}$ there exists $H_{0}$ of $\boldsymbol{H}$ such that

(2) $H_{0}^{-1} Z^{-1} x=x_{0}$.

By (1), (2) $\varphi\left(Z H_{0}, H\right)=(\zeta, x)$. Thus $R \subseteq \varphi\left(\boldsymbol{G} \times \mathfrak{G}_{r}\right)$ is proved. As noted before, $\varphi$ is holomorphic and regular. While by an easy computation we have

$$
\varphi^{-1}(\varphi, H)=\{(Z \cdot(\sigma), H) ;(\sigma) \in \mathbb{W}\} .
$$

Therefore $\varphi$ is an $n$ !-fold convering onto $R$. Set $\check{D}=\varphi^{-1}\left(D_{r}\right)$. Then $\check{D}$ is a real submanifold of $\boldsymbol{G} \times \mathfrak{h}_{r}$ which is in local holomorphically equivalent to $D_{r}$ since $\varphi$ is holomorphic. Thus we may consider our problem for $\breve{D}$ instead of $D_{r}$.

Now let us determine $\check{D}$ explicitly. Suppose $(Z, H) \in \check{D}$. By Linear Algebra $Z$ can be written uniquely as follows

$$
Z=A P
$$

where $A \in \boldsymbol{K}, P$ is a positive definite hermitian matrix. Then by the difinition of $D_{0}$ we have 


$$
\begin{aligned}
0 & =\frac{1}{i}\left(Z H Z^{-1}-{ }^{t} \overline{\left(Z H Z^{-1}\right)}\right)-Z x_{0}{ }^{t} \bar{x}_{0}{ }^{t} \bar{Z} \\
& =A\left(\frac{1}{i}\left(P H P^{-1}-P^{-1} H P\right)-P x_{0}{ }^{t} \bar{x} P\right) A^{-1} .
\end{aligned}
$$

Thus

$$
P H P^{-1}-P^{-1} H P=i P x_{0}{ }^{t} \bar{x}_{0} P,
$$

or equivalently

$$
H P^{-2}-P^{-2} H=i x_{0}{ }^{t} \bar{x}_{0} .
$$

Hence, if we set $Q=P^{-2}$, we have

$$
\left(h_{j}-\bar{h}_{k}\right) Q_{j k}=i \quad(j, k=1,2, \cdots, n)
$$

where

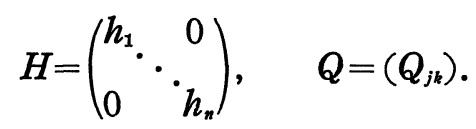

In particular because of the positive definiteness we obtain

$$
\text { (3) } \quad \operatorname{Im} h_{j}>0 \quad(j=1,2, \cdots, n) \text {. }
$$

Then automatically

$$
h_{j}-\bar{h}_{k} \neq 0 \quad(j, k=1,2, \cdots, n)
$$

and we get

$$
Q=\left(Q_{j k}\right)=\left(i /\left(h_{j}-\bar{h}_{k}\right)\right) \text {. }
$$

Conversely for $H \in \mathfrak{h}_{r}$ which satisfies (3), the matrix $\left(i /\left(h_{j}-\bar{h}_{k}\right)\right)$ is certainly positive-definite. For, determinants of its principal minors are all positive. This follows immediately from the following lemma

Lemma $\mathbf{H}_{2}$ (Hua [4]). Set $a_{j k}=\frac{1}{x_{j}+y_{k}}, a=\left(a_{j k}\right)$.

Then

$$
D(a)=\delta(x) \delta(y) \prod_{j, k=1}^{n} \frac{1}{x_{j}+y_{k}}
$$

where $\delta(x)=\prod_{j<k}\left(x_{j}-x_{k}\right)$.

Now define $Q(H)$ by setting

$$
Q(H)_{j k}=\frac{i}{h_{j}-\bar{h}_{k}}
$$


where $H=\left(\begin{array}{lll}h_{1} & & 0 \\ & \ddots & \\ 0 & & h_{n}\end{array}\right) \in \mathfrak{h}_{r} \operatorname{Im} h_{j}>0(j=1,2, \cdots, n)$, further set

$$
P(H)=Q(H)^{-1 / 2} \text {. }
$$

$P(H)$ is well-defined since $Q(H)$ is positive definite. Then we have from above argument

$$
\check{D}=\{(A P(H), H): A \in K H \in \check{\mathfrak{h}}\}
$$

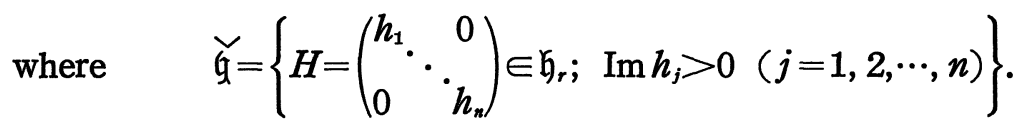

Define a real analytic diffeomorphism $\psi$ of $\boldsymbol{G} \times \check{\mathfrak{h}}$ onto itself by setting

$$
\psi(Z, H)=(Z P(H), H) \quad Z \in G, H \in \check{\mathfrak{h}} .
$$

Further set $\bar{\psi}=\left.\psi\right|_{\boldsymbol{K} \times \overline{\mathrm{h}}}$. Then $\bar{\psi}$ is a real analytic diffeomorphism of $\boldsymbol{K} \times \check{\mathfrak{h}}$ onto $\check{D}$.

4. 2. Solution of Problem $H$ for $D_{0}$. Let $\omega^{\prime}$ denote the MaurerCartan form of $\mathfrak{h}$ when $\mathfrak{h}$ is regarded as an abelian Lie group. Define an endomorphism $f_{(Z, H)}$ of $\mathcal{G}+\mathfrak{h}$ for any $(Z, H) \in \boldsymbol{G} \times \mathfrak{h}$ so that the diagram

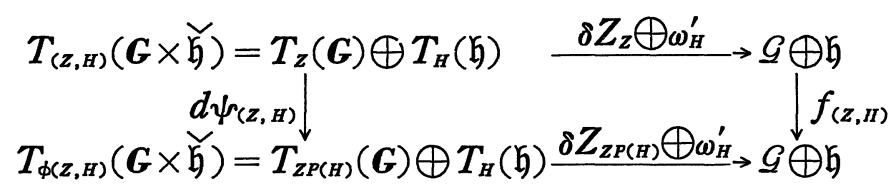

is commutative. To determine $f_{(z, H)}$ denote by $f_{H}$ the linear mapping of $\mathfrak{h}$ into $\mathcal{G}$ so that the following diagram is commutative:

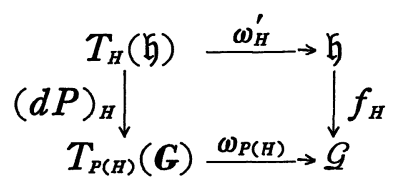

and set $Z_{1}=Z P(H)$. Then

$$
d Z_{1}=(d Z) P(H)+Z d P(H) .
$$

Therefore

$$
\delta Z_{1}=Z_{1}^{-1} d Z_{1}=a d\left(P(H)^{-1}\right) \delta Z+\delta P(H)
$$


Thus

$$
f_{(z, H)}=a d\left(P(H)^{-1}\right)+f_{H}+i d_{\mathfrak{h}} .
$$

From this it follows that for $A \in \boldsymbol{K}, H \in \mathfrak{h}$,

(4) $\omega_{A P(H)} \oplus \omega_{H}^{\prime}\left(T_{\psi(A, H)}(\check{D})\right)$

$$
=\left\{\left(\operatorname{ad}\left(p(H)^{-1}\right)(B)+f_{H}(L), L\right) ; B \in \mathcal{K} \quad L \in \mathfrak{h}\right\} .
$$

Denote by $\mathcal{G}^{(0,1)}$ the subspace of the complexification $\mathcal{G}^{\boldsymbol{c}}=\mathcal{G}+i \mathcal{G}$ of $\mathcal{G}$ given by

$$
\mathcal{G}^{(0,1)}=\{Z+i I Z, Z \in \mathcal{G}\}
$$

where $I$ is the linear isomorphism of $\mathcal{G}$ which defines the complex structure of $\mathcal{G}$. Then we have

$$
\begin{aligned}
& \mathcal{G}^{(0,1)} \cap \mathcal{K}^{\boldsymbol{c}}=(0) \\
& \mathcal{G}^{(0,1)}+\mathcal{K}^{\boldsymbol{c}}=\mathcal{G}^{\boldsymbol{c}} .
\end{aligned}
$$

Thus by (4), for $H \in \mathfrak{h}$ and for $L \in \mathfrak{h}^{(0,1)}$ there exists a unique $B(L, H)$ of $\mathcal{K}^{c}$ such that

$$
\left.d \psi_{(A, H)}(\widehat{B(L, H})_{A}+\widetilde{L}_{H}\right) \in T_{(A, H)}^{(0,1)}(G \times \mathfrak{h}) \cap T_{\psi(A, H)}^{C}(\check{D})
$$

where $\widetilde{L}$ denotes the vector field on $\mathfrak{h}$ such that $\omega^{\prime}(\widetilde{L})=L$ and $\mathfrak{h}^{(0,1)}$ is the subspace similar to $\mathcal{G}^{(0,1)}$ for $\mathfrak{h}$ instead of $\mathcal{G}$. In other words we have

Theorem 4. 2.1. Let $L$ be an element of $\mathfrak{h}^{(0,1)}$. Then there exists a unique typical vector field $X(L)$ on $M=K \times \check{\mathfrak{h}}$ such that $X(L)^{h}=\widetilde{L}$ and $d \bar{\psi}(X(L))$ is a section of the tangential Cauchy-Riemann bundle of $\check{D}$.

Here we have used word 'typical' setting $\Omega=\breve{\mathfrak{h}}$ in the preceding section. From this theorem we deduce

Corollary 4.2.1. Under the notation of §3 the following system of differential equations is equivalent to the Cauchy-Riemann equation $\bar{\partial} \boldsymbol{u}=0$ of $\boldsymbol{G} \times \mathfrak{h}:$

$$
d \psi\left(\widehat{X}\left(\frac{\partial}{\partial \bar{h}_{1}}\right)\right) u=0, \cdots, d \psi\left(\widehat{X}\left(\frac{\partial}{\partial \bar{h}_{n}}\right)\right) u=0 \quad \frac{\partial}{\partial \bar{Z}_{j k}} u=0 \quad(1 \leq j, k \leq n) .
$$


Here $\left(h_{1}, \cdots, h_{n}\right)$ is a system of coordinates of $\mathfrak{h}$ and $\left(Z_{j k}\right)$ is the system of coordinates of $\boldsymbol{G}$ as defined before.

Now let $\Omega$ be a domain of $\check{\mathfrak{h}}$, then we have an approximation theorem for $\mathcal{S}$-holomorphic functions on $\check{D}_{\Omega}=\psi(K \times \Omega)$. For this purpose we have to extend mappings of $C\left(M_{\Omega}\right)\left(M_{\Omega}=\boldsymbol{K} \times \Omega\right) f \rightarrow f_{\rho}, f \rightarrow f_{\rho}^{*}$ to mappings of $C\left(\check{\boldsymbol{D}}_{\Omega}\right)$ by $\psi$ and $\bar{\psi}$, i.e., we set for $f \in C\left(\check{D}_{\Omega}\right)$

$$
\begin{aligned}
& \left.f_{\rho}=\left(\bar{\psi}^{-1}\right) *\left(\bar{\psi}^{*} f\right)_{\rho}\right) \\
& f_{\rho}^{*}=\left(\left(\left.\boldsymbol{\psi}\right|_{\boldsymbol{G}_{\rho} \times \Omega}\right)^{-1}\right)^{*}\left(\left(\bar{\psi}^{*} f\right)_{\rho}^{*}\right) .
\end{aligned}
$$

Here $f_{\rho}^{*}$ is a function on $\widehat{M}_{\Omega, \rho}=G_{\rho} \times \Omega$.

Lemma 4.2.1. Let $f$ be an $S$-holomorphic function on $\check{D}_{\Omega}$, then $f_{\rho}^{*}$ is holomorphic in $\psi\left(\widetilde{M}_{\Omega, \rho}\right)$.

Proof. By Theorem 4.2.1, Corollary 4.2.1, Theorem 3.2.1 and the definition of $\mathcal{S}$-holomorphicity we have

$$
\bar{\partial} f_{\rho}^{*}=0
$$

in the distribution sense. Since $\bar{\partial}$ is elliptic, $f_{\rho}^{*}$ is holomorphic.

In view of this lemma Theorem 3.1.2' implies

Q.E.D.

Theorem 4.2.2. In the closed subspace of S-holomorphic functions on $\check{D}_{\Omega}$ of $C\left(\check{D}_{\Omega}\right)$ the set of restrictions of holomorphic functions near $\breve{D}_{s}$ is dense with respect to the topology induced from $C(M)$.

Now define an action of $\boldsymbol{K}$ on $\mathcal{G} \times V$ setting

$$
\rho_{0}(A)(Z, x)=\left(A Z A^{*}, A x\right) \quad A \in \boldsymbol{K},(Z, x) \in \mathcal{G} \times V .
$$

Then $D_{0}, D_{r}$ are $\boldsymbol{K}$-stable. Note that

$$
\varphi \circ L_{A}=\rho_{0}(A) \circ \varphi \text {. }
$$

Thus every $K$-stable open subset $S$ of $D_{r}$ is given by $S=\varphi\left(\check{D}_{\Omega}\right)$ for some open subset $\Omega$ of $\check{\mathfrak{h}}$. Therefore, for any $K$-stable open subset $S$ of $D_{r}$ the set of restrictions to $S$ of holomorphic functions near $S$ is dense in the space of $S$-holomorphic functions on $S$, the same is true for $p \cdot S=\rho(p) S\left(p \in D_{0}\right)$, $\rho$ being defined in $\$ 2.2$.

But the system of open subsets of $D_{0}$ 


$$
\Pi=\left\{\rho(p)\left(D_{r} \cap B_{\delta}\right): p \in D_{0}, 0<\delta<+\infty\right\}
$$

is a complete system of neighbourhoods. This can be seen as follows: Although $0 \notin D_{r}$, we can choose a sequence $\left(p_{n}\right) n=1,2, \cdots$ of points of $D_{r}$ such that $p_{n} \in B_{1 / n}$ since $D_{r}$ is open and dense in $D_{0}$. Then $\left\{\rho\left(p_{n}^{-1}\right)\left(D_{r} \cap B_{1 / n}\right): n=1,2, \cdots\right\}$ is certainly a complete system of neighbourhoods of 0 . To see this note that for any $\delta>0$ there exists a positive integer $n$ such that

$$
\left(B_{1 / n} \cap D_{0}\right)^{-1}\left(B_{1 / n} \cap D_{0}\right) \subset B_{\delta} \cap D_{0}
$$

because $D_{0}$ is a group manifold. Consequently for any $\delta>0$ we can choose $n \in N$ so that

$$
\rho\left(p_{n}^{-1}\right)\left(D_{r} \cap B_{1 / n}\right) \subset B_{\delta} \cap D_{0} .
$$

Thus our assertion is proved, and hence even

$$
\left\{\rho\left(p p_{n}^{-1}\right)\left(D_{r} \cap B_{1 / n}\right): p \in D_{0}, n=1,2, \cdots\right\}
$$

is a complete system of neighbourhoods of $D_{0}$.

Therefore we conclude that there exists a complete system of neighbourhoods $I$ such that for $S \in \Pi \Gamma_{S}\left(\mathscr{H}_{\mathcal{S}}\right)=H(S)$ where $\mathscr{H}_{\mathcal{S}}$ denotes the sheaf of germs of $\mathcal{S}$-holomorphic functions, i.e., the solution sheaf of the tangential Cauchy Riemann equation. Thus we have proved that $\mathscr{H}_{\mathcal{S}}=\mathcal{H}$. Combining this with Theorem 2.2.1 we obtain

Theorem 4. 2. $2^{\prime}$.

$$
\Gamma_{S}\left(\mathcal{H}_{\mathcal{S}}\right)=\Gamma_{S}(\mathcal{H})=\underset{\leftarrow}{H}(S)=H_{D}(S)=\left.H\left(U_{1}(S)\right)\right|_{s}
$$

for any open subset $S$ of $D_{0}$.

\section{§5. Application to Standard Real Submanifold of Second Kind}

5. 1. Basic Notations and Definitions. Let $V$ be an $n$-dimensional complex Hilbert space and $H(V)$ the space of hermitian forms on $V^{*}$. In $\S 1, V^{*}$ was considered to be $\operatorname{Hom}_{\boldsymbol{R}}(V, \boldsymbol{R})$. However $\operatorname{Hom}_{\boldsymbol{R}}(V, \boldsymbol{R})$ can be canonically identified with $\operatorname{Hom}_{C}(V, C)$ by the map

$$
\operatorname{Hom}_{\boldsymbol{R}}(V, \boldsymbol{R}) \ni f \rightarrow \frac{1}{2}(f-i f \circ I) \in \operatorname{Hom}_{\boldsymbol{C}}(V, \boldsymbol{C}) .
$$


Here $I$ denotes the automorphism of $V$ defined by $I(x)=i x(x \in V)$.

Now we shall prove the equivalence of $D_{0}$ with $M^{0}$. Recall that standard real submanifold $M^{0}$ in $N^{0}=V \times H(V)^{c}$ is given by

$$
M^{0}=\left\{(x, a) \in V \times H(V)^{c} ; \operatorname{Im}(\alpha)-\frac{1}{8}(x \otimes x+I x \otimes I x)=0\right\} .
$$

Here $\operatorname{Im}(\alpha)$ is the element of $H(V)$ such that $\alpha-i \operatorname{Im}(\alpha) \in H(V)$, that is, $\operatorname{Im}(\alpha)=\frac{1}{2 i}\left(\alpha-\alpha^{*}\right)$ where ${ }^{*}$ indicates the conjugation of $H(V)^{c}$ fixing $H(V)$. Consider the linear isomorphism $\pi_{0}$ of $\mathcal{G}_{h}$ onto $H(V)$ defined by

$$
\pi_{0}(A)\left(\operatorname{Re} x^{*}, \operatorname{Re} y^{*}\right)\left(=\left\langle\pi_{0}(A), \operatorname{Re} x^{*} \otimes \operatorname{Re} y^{*}\right\rangle\right)=\frac{1}{4} \operatorname{Re}(A x, y) \quad A \in \mathcal{G}_{k}
$$

where we have used the notations in $\S 2$. Computing directly we obtain

$$
\pi_{0}\left(x \otimes x^{*}\right)=\frac{1}{4}(x \otimes x+I x \otimes I x) .
$$

Let $\tilde{\pi}_{0}$ be the linear isomorphism of $\mathcal{G} \times V$ onto $N^{0}$ defined by $\tilde{\pi}_{0}(A, x)$ $=\left(x, \pi_{0}^{c}(A)\right)$. Then $\tilde{\pi}$ maps $D_{0}$ onto $M^{0}$. The image of the Siegel domain $D$ by $\tilde{\pi}_{0}$ is the domain

$$
\boldsymbol{D}=\left\{(x, \alpha): \operatorname{Im}(\alpha)-\frac{1}{8}(x \otimes x+I x \otimes I x)>0\right\} .
$$

Note that $M^{0}, \boldsymbol{D}$ are obtained once the complex structure of $V$ is given, i.e., they do not depend on the inner product on $V$.

Now let $\pi$ be a linear mapping of $H(V)$ onto a real vector space $W$. Then we can construct the furdamental Lie algebra $\mathfrak{m}(\pi)$ and the corresponding standard real submanifold $M(\pi)$ as in $\S 1$. Further consider the canonical linear map $\tilde{\pi}$ of $N^{0}=V+H(V)^{\circ}$ onto $N(\pi)=V+W^{c}$. Then $\hat{\pi}=\left.\tilde{\pi}\right|_{M^{0}}$ is the canonical homomorphism. In what follows we shall identify $D, D_{0}$ with $\boldsymbol{D}, M^{0}$ respectively by the map $\tilde{\pi}_{0}$, and use rather $D, D_{0}$ instead of $\boldsymbol{D}, M^{0}$ to avoid inessential complication of notaion. For example, $\hat{\pi}, \tilde{\pi}$ should be interpreted as the maps from $D_{0}, \mathcal{G} \times V$ onto $M(\pi), N(\pi)$ respectively. Under this convention the image by $\tilde{\pi}$ of $D$ is denoted by $D(\pi) . \quad D(\pi)$ also does not depend on the inne: product on $V$.

We shall now introduce function spaces and sheaves for $M(\pi)$ 
which are similar to those for $D_{0}$. For a subset $S$ of $M(\pi), H^{\circ}(S)$ is the set of restrictions to $S$ of holomorphic functions defined near $S$. In case $S$ is $\sigma$-compact, $H(S)$ is the closure of $H^{\circ}(S)$ in $C(S)$. For an open subset $S$ of $M(\pi), \underset{\leftarrow}{H}(S)$ is the set $\left\{f \in C(S) ;\left.f\right|_{s^{\prime}} \in H\left(S^{\prime}\right)\right.$ for any relatively compact subset $S^{\prime}$ of $\left.S\right\} . \mathcal{G}$ is the sheaf induced from the presheaf given by the system $(H(S))_{s: o p e n}$ with natural restriction maps. For a subset $U$ of $\overline{D(\pi)}$ such that $U \cap D(\pi)$ is a nonempty open subset and such that $U \subset \overline{U \cap D(\pi)}, \widetilde{H}(U)$ is the set $\left\{u \in C(U) ;\left.u\right|_{U \cap D(\pi)}\right.$ is holomorphic $\}$. Here the second condition on $U$ means that every continuous function on $U$ is determined by its restriction on $U \cap D(\pi)$. Further we set for any open subset $S$ of $M(\pi)$

$$
U_{\pi}(S)=\tilde{\pi}\left(U\left(\hat{\pi}^{-1}(S)\right)\right)
$$

where $U\left(\hat{\pi}^{-1}(S)\right)$ has appeared already in Lemma 2.2.10. $U_{\pi}(S)$ satisfies condition $U_{\pi}(S) \subset \overline{U_{\pi}(S) \cap D(\pi)}$ and $U_{\pi}(S) \cap D(\pi)$ is open. The first assertion follows immediately from

$$
U\left(\hat{\pi}^{-1}(S)\right) \subset \overline{U\left(\hat{\pi}^{-1}(S)\right) \cap D}
$$

which is obvious since $U\left(\hat{\pi}^{-1}(S)\right)$ is an open subset of $\bar{D}$. The second follows from

Lemma 5.1.1. If $U$ is an open subset of $\bar{D}$, then $\tilde{\pi}(U) \cap D(\pi)$ $=\tilde{\pi}(U \cap D)$.

Proof. Suppose $x \in \tilde{\pi}(U) \cap D(\pi)$. Then there are $p \in U, q \in D$ such that $\tilde{\pi}(p)=\tilde{\pi}(q)=x$. Set for $0 \leq t \leq 1$

$$
p(t)=(1-t) p+t q .
$$

Then $\mathfrak{l}(p(t))=(1-t) \mathfrak{l}(p)+t \mathfrak{l}(q)>0$ when $0<t \leq 1$. For $\mathfrak{l}(p) \geq 0$ and $\mathfrak{l}(q)>0$. But $U$ is open in $\bar{D}$, herce there exists $\delta>0$ such that $p(t) \in U$ for $0 \leq t \leq \delta$. Thus $p(t) \in U \cap D$ for $0<t<\delta$. Therefore $x=\tilde{\pi}(p)=\tilde{\pi}(p(t)) \in \tilde{\pi}(U \cap D)$. We have thus showed $\tilde{\pi}(U) \cap D(\pi)$ $\subset \tilde{\pi}(U \cap D)$. Since the opposite inclusion is obvious, the proof is complete.

We shall now define two important classes of standard real submanifolds which are the main objects in this paper. The standard real submanifold $M(\pi)$ is said to be totally indefinite or $\forall$-indefinite if 
every $0 \neq \alpha \in W^{*}$ the symmetric bilinear form

$$
\alpha(x, I y) \quad x, y \in V
$$

is indefinite. If every non-zero element of $\operatorname{Ker} \pi$ is indefinite, then $M(\pi)$ is dually $\forall$-indefinite. This terminology derives its origin from the fact that we can assign its dual for every $M(\pi)$ so that $M(\pi)$ is $\forall$-indefinite if and only if the dual is dually $\forall$-indefinite. This is done as follows. Let $K_{d}$ be the image of the transposed of $\pi$ and $\pi_{d}$ the canonical projection from $H\left(V^{*}\right)$ onto $W_{d}=H\left(V^{*}\right) / K_{d}$. Then $\pi_{d}$ gives rise to a standard real submanifold $M\left(\pi_{d}\right)$ in complex vector space $N\left(\pi_{d}\right)=V^{*} \times\left(W_{d}\right)^{c} . \quad M\left(\pi_{d}\right)$ is the dual of $M(\pi)$. Note here that the dual of the dual does not coincide with the original one in general. In fact $\left(\pi_{d}\right)_{d}$ is the projection of $H(V)$ onto $H(V) / \operatorname{Ker} \pi$. However we may consider that $M(\pi), M\left(\left(\pi_{d}\right)_{d}\right)$ are essentially the same. For, they are mutually equivalent by the canonical map $\tilde{\rho}$ induced from the commutative diagram

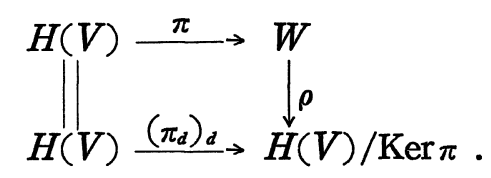

Lemma 5.1.2. $M(\pi)$ is $\forall$-indefinite if and only if $M\left(\pi_{d}\right)$ is dually $\forall$-indefinite.

This follows immediately from

Lemma 5.1.3. For a subspace $L$ of $H(V)$ the following statements are mutually equivalent:

(i) L contains a (positive) definite element.

(ii) $L^{\perp}=\left\{r \in H\left(V^{*}\right) ;\langle r, \eta\rangle=0 \quad{ }_{\eta} \in L\right\}$ contains no semidefinite element except for 0 .

Here $\langle$,$\rangle is the bilinear form on H(V) \times H\left(V^{*}\right)$ which gives the canonical identification of $H\left(V^{*}\right)$ with $H(V)^{*}$. For the proof of this see L. L. Dines [1] or Lemma 3 of I. Naruki [7]. Note that, in this notation, $\operatorname{Ker} \pi_{d}=(\operatorname{Ker} \pi)^{\perp}$, in view of which Lemma 5.12 is obvious. If $M(\pi)$ is either $\forall$-indefinite, or dually $\forall$-indefinite, then $M(\pi)$ is 
said to be stable. The standard real submanifold which is not stable is called unstable one. The meaning of the word is the following: Let $G_{k}$ denote the Grassmann manifold consisting of all $k$-dimensional subspaces of $H(V)$. Given an $H \in G_{k}, M_{H}$ denotes the standard real submanifold induced from the canonical map $\pi_{H}: H(V) \rightarrow H(V) / H$. Then the set $\left\{H \in G_{k}: M_{H}\right.$ is unstable $\}$ is closed in $G_{k}$ and of the first category, i. e., without interior, while the sets $\left\{H \in G_{k}: M_{H}\right.$ is $\forall$-indefinite $\},\left\{H \in G_{k}: M_{H}\right.$ is dually $\forall$-indefinite $\}$ are open in $G_{k}$. Thus one may say that almost all standard real submanifolds are stable.

In this paper we shall only concern ourselves with stable ones and the rain objective is to show

$$
\stackrel{H}{\leftarrow}(S)=\Gamma_{\mathrm{s}}(\mathscr{H})=\Gamma_{s}\left(\mathscr{H}_{S}\right)=\left.\widetilde{H}\left(U_{\pi}(S)\right)\right|_{s}
$$

when $S$ is an open subset of a stable standard real submanifold. The proof is given first for $\forall$-indefinite case and next for dually $\forall$-indefinite case.

5. 2. $\forall$-indefinite Case. In this case the study is based on the following

Lemma 5.2.1. If $M(\pi)$ is $\forall$-indefinite, then $N(\pi)=\tilde{\pi}(D)=D(\pi)$.

Proof. It suffices to show $D+(\operatorname{Ker} \pi)^{c}=N^{0}=V \lambda^{\prime} H(V)^{C} . \quad$ By assumption and Lemma 5.1.3, Ker $\pi$ contains a positive definite element $r_{0}$ of $H(V)$. But, for any $p \in N^{0} \mathfrak{I}(p)+t r_{0}$ is positive definite for sufficiently large $t>0$, i.e., $p+i t r_{0}$ belongs to $D$. Hence $p \in D+i \operatorname{Ker} \pi$. Thus we have proved $N^{0}=D+i \operatorname{Ker} \pi=D+(\operatorname{Ker} \pi)^{c}$.

Q.E.D.

Now we shall prove a stronger version of the statement announced at the end of 5.1 for totally indefinite $M(\pi)$.

Theorem 5.2.1. If $S$ is an open subset of an $\forall$-indefinite $M(\pi)$, then $U_{\pi}^{1}(S)$ is an open subset of $N^{\prime}(\pi)$ and

$$
\underset{\leftarrow}{H}(S)=\Gamma_{s}(\mathcal{I l})=\Gamma_{s}\left(\mathcal{H}_{\mathcal{S}}\right)=\left.H\left(U_{\pi}^{1}(S)\right)\right|_{s}=\left.H^{\circ}\left(U_{\pi}^{1}(S)\right)\right|_{s} .
$$

Here we have put $U_{\pi}^{1}(S)=\tilde{\pi}\left(U_{1}\left(\hat{\pi}^{-1}(S)\right)\right)$.

Proof. Suppose $f \in \Gamma_{S}\left(\mathcal{H}_{\mathcal{S}}\right)$. Then $\hat{\pi}^{*}(f) \in \Gamma_{\mathcal{S}^{\prime}}\left(\mathscr{H}_{\mathcal{S}}\right)$ where, for 
simplicity, we have set $S^{\prime}=\hat{\pi}^{-1}(S)$. From Theorem 4.2.2' it follows the existence of $u \in \widetilde{H}\left(U_{1}\left(S^{\prime}\right)\right)$ such that $\left.u\right|_{s^{\prime}}=\hat{\pi}^{*}(f)$. Note that both $\hat{\pi}^{*}(f)$ and $S^{\prime}$ are invariant under translations parallel to Ker $\pi$. Thus Lemma 2.2.11 implies that there exists a function $v$ on $U_{\pi}^{1}(S)$ $=\tilde{\pi}\left(U_{1}\left(S^{\prime}\right)\right)$ such that

$$
v(\tilde{\pi}(p))=u(p) \quad p \in U_{1}\left(S^{\prime}\right) .
$$

Note that the combination of Lemmas 5.1.1 and 5.2.1 implies that $\left.\tilde{\pi}\right|_{D}$ is open. Thus the continuity of $v$ is obvious.

On the other hand we have

$$
\tilde{\pi}\left(U_{1}\left(S^{\prime}\right)\right)=\tilde{\pi}\left(U_{1}(S)\right) \cap D(\pi)=\tilde{\pi}\left(U_{1}(S) \cap D\right)
$$

where we have used Lemma 5.2.1 in the first step and Lemma 5.1.1 in the last. This implies that $U_{\pi}^{1}(S)$ is open in $N(\pi)$. Furthermore, since $\left.u\right|_{U_{1}(S) \cup D}$ is holomorphic, $v$ is also holomorphic. Hence $f=\left.v\right|_{s}$ $\left.\in H\left(U_{\pi}^{1}(S)\right)\right|_{s}=\left.H^{\circ}\left(U_{\pi}^{1}(S)\right)\right|_{s} . \quad$ Thus we have proved $\Gamma_{s}\left(\mathscr{H}_{\mathcal{S}}\right) \subset$ $\left.H\left(U^{1}(S)\right)\right|_{s}$ which, together with obvious inclusions $\Gamma_{s}\left(\mathscr{H}_{S}\right) \supset \Gamma_{S}(\mathscr{H})$

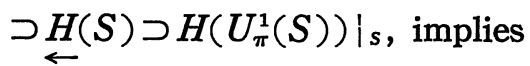

$$
\stackrel{H}{\leftarrow}(S)=\Gamma_{S}(\mathscr{H})=\Gamma_{S}\left(\mathscr{H}_{S}\right)=\left.H\left(U_{\pi}^{1}(S)\right)\right|_{s}=\left.H^{\circ}\left(U_{\pi}^{1}(S)\right)\right|_{s} .
$$

Q.E.D.

From this theorem it follows trivially

Corollary 5. 2.1. If $M(\pi)$ is $\forall$-indefinite, then every S-holomorphic function in an open set of $M(\pi)$ is real analytic.

Note that $U_{1}\left(D_{0}\right)=\bar{D}$ by the construction of $U_{1}(S)$. Therefore $U_{\pi}^{1}(M(\pi))=N(\pi)$ by Lemma 5.2.1. Thus we obtain

Corollary 5.2.2. Every S-holomorphic function on $M(\pi)$ is the restriction of an entire holomorphic function of $N(\pi)$ if $M(\pi)$ is $\forall$ indefinite.

By means of Theorem 5.2.1 we shall show that $\Gamma_{s}\left(\mathscr{H}_{\mathcal{S}}\right)$ is a Montel space.

Corollary 5. 2.3. Under the hypothesis of Theorem 5.2.1 the map 


$$
\Gamma_{s}\left(\mathscr{H}_{\mathcal{S}}\right) \ni f \mapsto \varphi f \in C_{0}^{\infty}(S)
$$

is a compact operator for $\varphi \in C_{0}^{\infty}(S)$.

Proof. Since the restriction map $r: H(U(S)) \rightarrow \Gamma_{S}\left(\mathscr{H}_{\mathcal{S}}\right)$ is a continuous bijection and since $\widetilde{H}(U(S)), \Gamma_{S}\left(\mathscr{H}_{S}\right)$ are Fréchet spaces, the inverse $\eta$ of $r$ is also continuous (by Banach's theorem). Note that $\widetilde{H}(U(S))=H(U(S))$ since $U(S)$ is an open set of $N(\pi)$. Therefore the map $\zeta$

$$
\widetilde{H}(U(S)) \ni u \mapsto \psi u \in C_{0}^{\infty}(U(S))
$$

is compact where $\psi$ is a function of $C_{0}^{\infty}(U(S))$ such that $\left.\psi\right|_{s}=\varphi$. Therefore the map

$$
\Gamma_{s}\left(\mathcal{H}_{\mathcal{S}}\right) \ni f \mapsto \varphi f=\left.\zeta \circ \eta(f)\right|_{s} \in C_{0}^{\infty}(S)
$$

is compact.

Q.E.D.

Now we shall extend this Corollary for a somewhat wider class of real submanifolds. Let $M$ be a real submanifold of a complex manifold $\widetilde{M}$. $\quad M$ is called a locally flat real submanifold of type $M(\pi)$ if for any $p$ of $M$ there exist a neighbourhood $U$ in $\widetilde{M}$ of $p$ and a biholomorphic mapping $\varphi$ of $U$ into $N(\pi)$ such that $\varphi(U \cap M)$ is open in $M(\pi)$.

Corollary 5. 2. 3'. Suppose that $M(\pi)$ is totally indefinite and $M$ is a locally flat real submanifold of type $M(\pi)$. Then the map

$$
\Gamma_{s}\left(\mathcal{H}_{\mathcal{S}}\right) \ni f \mapsto \varphi f \in C_{0}^{\infty}(S)
$$

is a compact operator for an open set $S$ of $M$ and $\varphi \in C_{0}^{\infty}(S)$.

This corollary implies the finiteness theorem for $\mathcal{S}$-holomorphic vector bundles on a compact locally flat real submanifold. Let $M$ be a real submanifold of a complex manifold such that the tangential Cauchy-Riemann bundle of $M$ is well-defined. A complex vector bundle $E$ on $M$ with a subsheaf $S$ of $E$ is called an $S$-holomorphic vector bundle on $M$ if, for any point $p$ of $M$, there exist a neighbourhood $U$ and sections $s^{1}, s^{2}, \cdots, s^{\mathfrak{l}}(\mathfrak{l}=$ fiber $\operatorname{dim} E)$ over $U$ of $S$ such that

$$
\sum_{k=1}^{l} f_{k} s^{k} \in \Gamma_{V}(S) \Longleftrightarrow f_{1}, \cdots, f_{\mathfrak{l}} \text { are } \mathcal{S} \text {-holomorphic in } U \text {. }
$$


By Corollary 5. 2. 3', we obtain

Proposition 5.2.1. Let $M$ be a compact locally flat real submanifold of type $M(\pi)$ where $M(\pi)$ is totally indefinite and $(E, S)$ an"S-holomorphic vector bundle over $M$. Then $\Gamma(S)$ is finite-dimensional.

Proof. Obvious since $\Gamma(S)$ is a locally compact Banach space by Corollary 5. 2. $3^{\prime}$.

This proposition is a special case of more general theorem of Naruki [7] proved by using the method of Hörmander. But the reason why we have proved Proposition 5.2.1 using the holomorphic extension is that the method of this paper suggests us a conjecture on holomorphic extension. Let $M$ be a real submanifold of a complex manifold for which Levi-Tanaka algebras are well-defined and let $\mathfrak{m}_{p}=\sum_{k=1}^{\mu} g_{p}^{k}$ be the Levi-Tanaka algebra of $M$ at $p$. Then $\mathrm{m}_{p}^{2}=\mathrm{g}_{p}^{1}+\mathrm{g}_{p}^{2}$ is also a fundamental Lie algebra under the convention that

$$
\left[g_{p}^{1}, g_{p}^{2}\right]=\left[g_{p}^{2}, g_{p}^{2}\right]=(0) .
$$

$M$ is called totally indefinite if for any $p \in M \mathfrak{m}_{p}^{2}$ is totally indefinite (i.e., the corresponding standard real submanifold $M\left(\mathfrak{m}_{p}^{2}\right)$ is totally indefinite). Now our conjecture is the following.

Conjecture: Let $M$ be a totally indefinite real submanifold of a complex manifold $M$. Then there exists a neighbourhood $\widetilde{U}$ in $\widetilde{M}$ of $M$ such that the restriction map

$$
H(\widetilde{U}) \rightarrow \Gamma_{M}\left(\mathscr{H}_{\mathcal{S}}\right)
$$

is onto.

If this conjecture is true, it will be almost obvious that the space of global sections of an $\mathcal{S}$-holomorphic vector bundle over a compact totally indefinite real submanifold is finite-dimensional, which is a main theorem of [7].

Remark. For any compact, locally flat and totally indefinite real subrnanifold $M S$-holomorphic functions are constant on each component of $M$. This can be proved as follows. Let $H$ be the vector space of 
$\mathcal{S}$-holomorphic functions on $M$. Then by Proposition 1 this is a finitedimensional algebra over $\boldsymbol{C}$. Therefore any element of $H$ satisfies an algebraic equation

$$
P(f)=0 \quad P \in C[x] .
$$

Suppose that $c_{1}, c_{2}, \cdots, c_{k}$ are the roots of $P$, then

$$
\left(f-c_{1}\right)\left(f-c_{2}\right) \cdots\left(f-c_{k}\right)=0 .
$$

Therefore $f$ takes only a finite number of values. Since $f$ is continuous, $f$ is constant on each component of $M$.

As an example, we shall now give a compact, $\forall$-indefinite and locally flat real submaifold, which is an orbit of a subgroup of the holomorphic transformation group on a complex Grassmann manifold.

Example (Tanaka). First we construct many linear groups:

$$
\begin{aligned}
& G=G L(m, C) \quad\left(m=n+2 n^{\prime} \quad n=p+q p, q>0\right) \\
& G^{0}=\left\{A \in G: A J^{t} \bar{A}=J\right\} \quad J=\left(\begin{array}{l|l|l|l} 
& & & -i I_{n^{\prime}} \\
\hline & I_{p} & & \\
\hline & & -I_{q} & \\
\hline i I_{n^{\prime}} & & &
\end{array}\right) \\
& L=\left\{A=\left(a_{i j}\right) \in G: a_{i j}=0 \text { if } n^{\prime}<i \leqq m, 1 \leqq j \leqq n^{\prime}\right\} \\
& G^{\prime}=G^{0} \cap L \text {. }
\end{aligned}
$$

Then the Lie algebras $\mathcal{G}^{0}, \mathcal{G}^{\prime}, \mathcal{L}$ of $G^{0}, G^{\prime}, L$ are given by

$$
\begin{aligned}
& \mathcal{G}^{0}=\left\{A \in M_{m}(\boldsymbol{C}): A J+J^{t} \bar{A}=0\right\} \\
& \mathcal{L}=\left\{A=\left(a_{i j}\right) \in M_{m}(\boldsymbol{C}): a_{i j}=0 \text { if } n^{\prime}<i \leqq m, 1 \leqq j<n^{\prime}\right\} \\
& \mathcal{G}^{\prime}=\mathcal{G}^{0} \cap \mathcal{L} .
\end{aligned}
$$

By direct calculation one sees that $\mathcal{G}^{0}$ is the set of matrices of the following form 


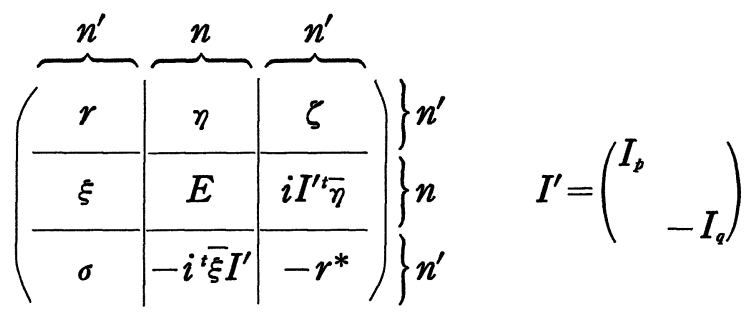

where $\sigma, \zeta$ are hermitian, $E I^{\prime}+I^{\prime} \bar{E}=0$. We shall set

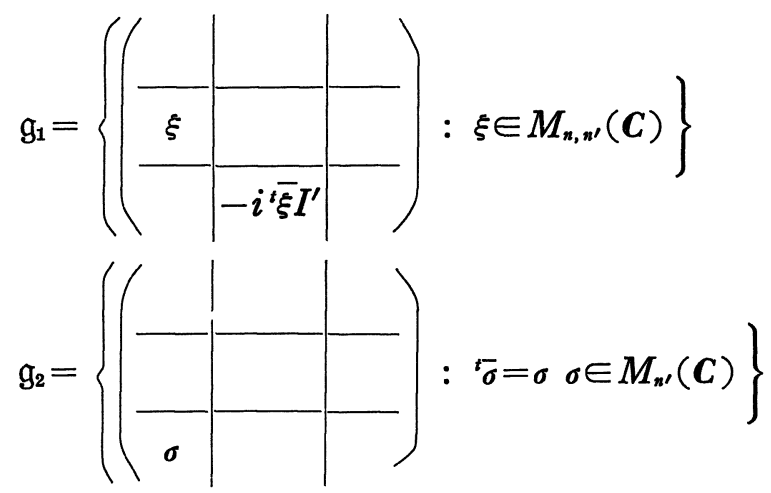

$$
\mathfrak{m}=\mathrm{g}_{1}+\mathrm{g}_{2} \text {. }
$$

Then $\mathfrak{m}$ is a subalgebra of $\mathcal{G}^{0}$ such that $\left[\mathfrak{g}_{1}, \mathfrak{g}_{1}\right] \subseteq \mathrm{g}_{2},\left[\mathfrak{g}_{2}, \mathfrak{g}_{2}\right]=$ $=0$. We shall make $g_{1}$ a complex vector space so that th mapping $\tau$

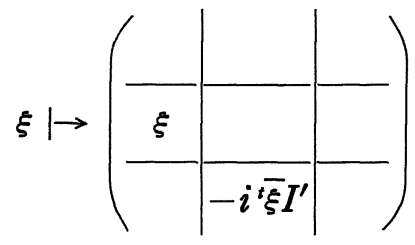

is a complex linear isomorphism of $M_{n, n^{\prime}}(C)$ onto $g_{1}$. Thus fundamental Lie algebra and $\mathrm{m}^{c}$ can be identified with the su of $M_{n}(C)$ consisting of matrices with the following form

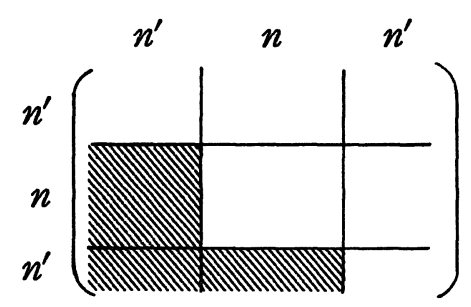


by the mappings

$$
\tau^{c}: g_{1}^{c} \ni \xi \otimes 1+\xi^{\prime} \otimes i \mid \rightarrow\left(\overline{\xi+i \xi^{\prime}}\left|\frac{}{\left({ }^{t} \overline{\xi^{\prime}}-i^{t} \bar{\xi}\right) I^{\prime}}\right|-\right)
$$

and

$$
\mathrm{g}_{2}^{C} \ni\left(\sigma \otimes 1+\sigma^{\prime} \otimes i\right) \mapsto\left(\frac{}{\sigma+i \sigma} \mid \square\right)
$$

The connected Lie subgroup of $G^{0}$ with Lie algebra $\mathfrak{m}$ (resp $\mathfrak{m}^{\boldsymbol{c}}$ ) shall be identified with $M^{\prime}\left(\operatorname{resp} M^{c}\right)$ introduced for $m$ in $\S 1$. Under this identification we have

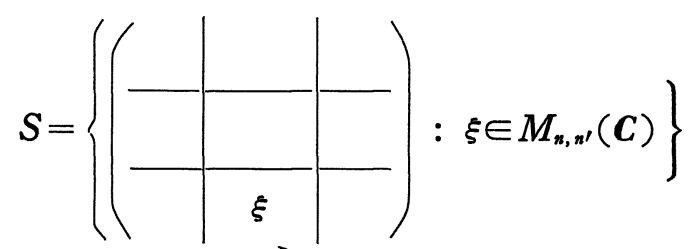

where $S$ is the abelian subalgebra of $\mathfrak{i n}$ introduced in $\S 1$. Denote by $H$ the Lie subgroup of $G$ with Lie algebra $S$.

$$
S=L \cap \mathfrak{m}^{c}, \quad H=L \cap M^{c} \text {. }
$$

Then the canonical map

$$
M^{c} / H \rightarrow G / L
$$

is one to one and regular (not onto).

On the other hand the canonical map

$$
M^{\prime} \rightarrow G^{0} / G^{\prime}
$$

is also one to one and regular. In fact $G^{\prime} \cap M^{\prime}=(e)$ and $\mathfrak{m}+\mathcal{G}^{\prime}=\mathcal{G}^{0}$. Moreover the following diagram is commutative

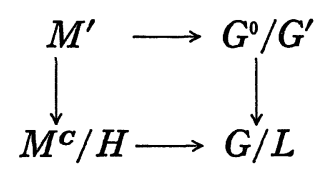


where all arrows are canonical. Thus $G^{0} / G^{\prime}$ contains a locally flat open subset. But the canonical left operation of $G^{0}$ on $G / L$ is holomorphic. Therefore $G^{0} / G^{\prime}$ itself is locally flat.

Now we take $K=U(m) \cap G^{0}$ as a maximal compact subgroup of $G^{0}$ and denote by $\mathcal{K}$ the Lie algebra of $K$. Then

$$
\mathcal{G}^{0}=\mathcal{K}+\mathcal{G}^{\prime} .
$$

Therefore orbits of $K$ in $G^{0} / G^{\prime}$ are open, and it is obviously compact. Since $G^{0} / G^{\prime}$ is connected, $K$ operates transitively on $G^{0} / G^{\prime}$, and $G^{0} / G^{\prime}$ itself is compact. Thus $G^{0} / G^{\prime}$ is a compact locally flat real submanifold of $G / L$. (Note that $G / L$ is a Grassmann manifold.)

In order to show that $G^{0} / G^{\prime}$ is totally indefinite one may investigate Lie algebra m. Recall that $g_{1}$ is isomorphic to $M_{n, n^{\prime}}(\boldsymbol{C})$ by $\tau$. Note that

$$
[\tau(\xi), I \tau(\eta)]={ }^{t} \bar{\xi} I^{\prime} \eta+{ }^{t} \bar{\eta} I^{\prime} \xi .
$$

We shall prove that for any non-zero hermitian $\left(n^{\prime}, n^{\prime}\right)$-matrix $A$, the symmetric form

$$
[A](\xi, \eta)=\operatorname{Sp}(A[\tau(\xi), I \tau(\eta)])
$$

is indefinite. Denote by $S_{k}$ the subspace of $M_{n, n}(\boldsymbol{C})$ of $k$-th column vectors and set $A=\left(a_{i j}\right)_{1 \leq i, j \leq n^{\prime}}$.

1) Suppose that $a_{i j} \neq 0$ for some $i \neq j$. For any $u \in S_{i}, v \in S_{j}$

$$
[A](u+v, u+v)=2 \operatorname{Re}\left(a\left(\sum_{k=1}^{p} u_{k} \bar{v}_{k}-\sum_{k=p+1}^{n} u_{k} \bar{v}_{k}\right)\right)
$$

where we set $a_{i j}=a$. Choosing a suitable $e^{i \Phi}$ so that $e^{i \Phi}$ a is real, we have

$$
[A]\left(e^{i \Phi} u+v, e^{i \Phi} u+v\right)=2 e^{i \Phi} a \operatorname{Re}\left(\sum_{k=1}^{p} u_{k} \bar{v}_{k}-\sum_{k=p+1}^{n} u_{k} \bar{v}_{k}\right)
$$

which can obviously be made positive as well as negative by taking suitable $u, v$.

2) Suppose $a=a_{i i} \neq 0$ for some $i$. Then, for $u \in S_{i}$

$$
[A](u, u)=2 a\left(\sum_{k=1}^{p} u_{k} \bar{u}_{k}-\sum_{k=p+1}^{n} u_{k} \bar{u}_{k}\right) .
$$

Thus the restriction of $[A]$ to $S_{i}$ is indefinite. 
To sum up we conclude that $G^{\circ} / G^{\prime}$ is a compact, locally flat and totally indefinite real submanifold of $G / L$.

5. 3. Dually $\forall$-indefinite CASE. As announced before the objective of this paragraph is to prove the following

Theorem 5. 3.1. If $M(\pi)$ is dually $\forall$-indefinite, then we have

$$
\underset{\leftarrow}{\leftarrow}(S)=\Gamma_{S}(\mathscr{H})=\Gamma_{s}\left(\mathscr{H}_{\mathcal{S}}\right)=\left.\widetilde{H}\left(U_{\pi}(S)\right)\right|_{s}
$$

for any open subset $S$ of $M(\pi)$.

To prove this we need a lemma concerning closed cones in real vector spaces.

Lemma 5.3.1. Let $K$ be a closed cone in a real vector space $W$, and $H$ be a subspace of $W$ such that $H \cap K=(0)$. Then the set $(B+H) \cap K$ is bounded if $B$ is a bounded set of $W$.

Proof. Choose a fixed norm \|\| on $W$. Suppose that $(B+H) \cap K$ is not bounded while $B$ is bounded. Then there exist sequences $\left\{x_{\nu}\right\}_{\nu=1}^{\infty} \subseteq B$ and $\left\{h_{\nu}\right\}_{\nu=1}^{\infty} \subset H \backslash\{0\}$ such that $\left\{x_{\nu}+h_{\nu}\right\}_{\nu=1}^{\infty} \subset K$ and such that $\left\|h_{\nu}\right\| \rightarrow \infty(\nu \rightarrow \infty)$. Set $\left\|h_{\nu}^{\prime}\right\|=\left\|h_{\nu}\right\|^{-1} h_{\nu}$. Since $\left\|h_{\nu}^{\prime}\right\|=1$, we may assume that $h_{\nu}^{\prime}$ converges to some $h_{0} \in H$ when $\nu \rightarrow \infty$. Then $\left\|h_{0}\right\|=1$, and further $\left\|h_{\nu}\right\|^{-1}\left(x_{\nu}+h_{\nu}\right) \rightarrow h_{0}(\nu \rightarrow \infty)$. On the other hand, since $\left\|h_{\nu}\right\|^{-1}\left(x_{\nu}\right.$ $\left.+h_{v}\right) \in K, h_{0}$ also belongs to $\bar{K}=K$. But then $H \cap K=(0)$ implies $h_{0}=0$, which, however, contradicts $\left\|h_{0}\right\|=1$. Thus $(B+H) \cap K$ is bounded.

Q.E.D.

Proof of Theorem 5.3.1. Suppose $f \in \Gamma_{s}\left(\mathscr{H}_{\mathcal{S}}\right)$. Set for simplicity $H=\operatorname{Ker} \pi, S^{\prime}=\hat{\pi}^{-1}(S)$. Then $\hat{\pi}^{*}(f) \in \Gamma_{S^{\prime}}\left(\mathscr{H}_{\mathcal{S}}\right)$. Moreover $\hat{\pi}^{*}(f)$ together with $S^{\prime}$ is invariant by translation parallel to $H$. Hence by Lemma 2.2.11 and Theorem 4.2.2 there exists a function $u$ on $U_{1}\left(S^{\prime}\right)+H^{c}$, such that $\left.u\right|_{s^{\prime}}=\hat{\pi}^{*}(f),\left.u\right|_{U_{1}\left(s^{\prime}\right)} \in \widetilde{H}\left(U_{1}\left(S^{\prime}\right)\right)$, and $\tau_{h}^{*}(u)=u$ $\left(h \in H^{c}\right)$. Here $\tau_{k}$ is the translation $p \rightarrow p+h\left(p \in N^{0}\right)$.

Now we shall prove the continuity of $v=\left.u\right|_{U\left(s^{\prime}\right)+H} c$. First recall that $U\left(S^{\prime}\right)+H=U\left(S^{\prime}\right)$, so that

$$
U\left(S^{\prime}\right)+H^{c}=U\left(S^{\prime}\right)+i H .
$$


Observe that the following statement is sufficient for $v$ to be continuous: From every sequence $\left\{x_{v}\right\}_{v=1}^{\infty} \subset U\left(S^{\prime}\right)+i H$ converging to $x_{0} \in U\left(S^{\prime}\right)$ $+i H$, it is possibie to choose a subsequence $\left\{x_{\nu_{k}}\right\}_{k=1}^{\infty}$ such that $u\left(x_{\nu_{k}}\right)$ $\rightarrow u\left(x_{0}\right)(k \rightarrow \infty)$. Let us prove this statement. Write each $x_{\nu}(\nu=0$, $1,2, \cdots)$ in the form $x_{\nu}=p_{\nu}+i h_{\nu}\left(p_{\nu} \in U\left(S^{\prime}\right), h_{\nu} \in H\right)$. Then $\mathfrak{I}\left(x_{\nu}\right)$ $=\mathfrak{l}\left(p_{\nu}\right)+h_{\nu}$ and $\mathfrak{l}\left(p_{\nu}\right) \geq 0$, that is, $\mathfrak{l}\left(p_{\nu}\right)$ lies in the closure $\bar{K}_{0}$ of the cone $K_{0}$ consisting of positive definite elements of $H(V)$. Here we have set as in $\S 2, \mathfrak{l}(x, \alpha)=\operatorname{Im}(\alpha)-\frac{1}{8}(x \otimes x+I x \otimes I x)$ for $(x, \alpha) \in V$ $\times H(V)^{c}$. By the assumption $H \cap \overline{K_{0}}=\operatorname{Ker} \pi \cap \bar{K}_{0}=(0)$. Hence Lemma 5.3.1 implies that $\left\{h_{\nu}\right\}_{\nu=1}^{\infty}$ is bounded. Thus there certainly exists a subsequence $\left\{h_{\nu_{k}}\right\}_{k=1}^{\infty}$ which converges to some $h_{0}^{\prime}$ of $H$. But then $\left\{p_{\nu_{k}}\right\}_{k=1}^{\infty}$ also converges to $p_{0}^{\prime}=x_{0}-i h_{0}^{\prime} \in \overline{U\left(S^{\prime}\right)}$. However $p_{0}^{\prime} \in \partial S^{\prime}$ implies that $0=\mathfrak{l}\left(p_{0}^{\prime}\right)=\mathfrak{l}\left(x_{0}\right)-h_{0}^{\prime}=\mathfrak{l}\left(p_{0}\right)+h_{0}-h_{0}^{\prime}$, from which $\mathfrak{l}\left(p_{0}\right)=h_{0}^{\prime}-h_{0} \in \bar{K}_{0} \cap H$ $=(0)$, that is, $p_{0}=p_{0}^{\prime} \in \partial S^{\prime}$, contradicting $p_{0} \in U\left(S^{\prime}\right)$. (Recall $S^{\prime}=U\left(S^{\prime}\right)$ $\cup D_{0}$.) Thus $p_{0}^{\prime} \in \overline{U\left(S^{\prime}\right)} \backslash \partial S$, which, according to Lemma 2.2.10, implies $p_{0}^{\prime} \in U_{1}(S)$. Since $u$ is continuous in $U_{1}(S)$ it follows

$$
u\left(x_{v}\right)=u\left(p_{v}\right) \rightarrow u\left(p_{0}^{\prime}\right)=u\left(x_{0}\right)
$$

when $\nu \rightarrow \infty$. The required statement is thus proved, hence $v$ is continuous. Recall now that $u$, hence also $v$ are invariant by $\tau_{h}\left(h \in H^{c}\right)$. Thus there exists a continuous function $w$ on $U_{\pi}(S)=\tilde{\pi}\left(U\left(S^{\prime}\right)\right)$ such that $w(\tilde{\pi}(p))=v(p) \quad p \in U\left(S^{\prime}\right)$. The function $w$ is certainly holomorphic in $U_{\pi}(S) \cap D(\pi)$, for $U_{\pi}(S) \cap D(\pi)=\tilde{\pi}\left(U\left(S^{\prime}\right) \cap D\right)$ by Lemma 5.1.1 and $v$ is holomorphic in $U\left(S^{\prime}\right) \cap D$. Thus $f=\left.\left.w\right|_{s} \in H\left(U_{\pi}(S)\right)\right|_{s}$. And thus we have proved

$$
\left.\Gamma_{s}\left(\mathscr{H}_{\mathcal{S}}\right) \subseteq \widetilde{H}(U(S))\right|_{s} .
$$

Now let $\gamma_{0}$ be a positive definite form in $H(V)$ and $S^{\prime}$ a relatively compact subset of $S$. Then there exists a relatively compact subset $S^{\prime \prime}$ of $\hat{\pi}^{-1}(S)$ such that $\hat{\pi}\left(S^{\prime \prime}\right)=S^{\prime}$. Since $U\left(\hat{\pi}^{-1}(S)\right)$ is open in $\bar{D}$, $S^{\prime \prime}+i t_{r_{0}}$ is contained in $U\left(\hat{\pi}^{-1}(S)\right) \cap D$ for sufficiently small $t>0$. Thus, for a sufficiently small $t>0$,

$$
S^{\prime}+i t \pi\left(\gamma_{0}\right) \subseteq U(S) \cap D(\pi) .
$$

Therefore, arguing as in the proof of Lemma 2.2.9, we obtain 


$$
\left.\widetilde{H}(U(S))\right|_{s} \subseteq H(S) .
$$

In view of obvious inclusions $\underset{\leftarrow}{H}(S) \subseteq \Gamma_{s}(\mathcal{H}) \subseteq \Gamma\left(\mathcal{H}_{\mathcal{S}}\right)$, (1) and (2) imply

$$
\underset{\leftarrow}{H}(S)=\Gamma_{s}=\left(\mathcal{H}_{\mathcal{S}}\right)=\left.\widetilde{H}(U(S))\right|_{s} .
$$

Here it should be remarked that $\tilde{\pi}(\bar{D})=\overline{D(\pi)}$ when $M(\pi)$ is dually $\forall$-indefinite. This follows from

$$
\begin{aligned}
& \bar{D}=\left\{(x, \alpha): \mathfrak{l}(x, \alpha) \in \overline{K_{0}}\right\} \\
& \overline{D(\pi)}=\left\{\tilde{\pi}(x, \alpha) ; \pi(\mathfrak{l}(x, \alpha)) \in \overline{\pi\left(K_{0}\right)}\right\} \\
& \pi\left(K_{0}\right)=\overline{\pi\left(K_{0}\right)} .
\end{aligned}
$$

The last relation is an immediate consequence of Lemma 5.3.1.

Note that $U_{\pi}(S)$ never swells up to $\overline{D(\pi)}$ even if $S=M(\pi)$. However we can prove the result parallel to Corollary 5.2.2 also in the present case.

Theorem 5.2.2. If $M(\pi)$ is dually $\forall$-indefinite, then the restriction map $\widetilde{H}(\overline{D(\pi)}) \rightarrow \Gamma\left(\mathcal{I}_{\mathcal{S}}\right)$ is a topological isomorphism.

For the proof, first note that $U_{1}\left(D_{0}\right)=\bar{D}$, next use the relation $\overline{U_{1}\left(D_{0}\right)}=U_{1}\left(D_{0}\right)$ instead of $\overline{U(S)} \backslash \partial S \subset U_{1}(S)$. Then the proof of Theorem 5.3.1 can be applied without further change.

Now we shall show that the maximal open subset of $\overline{D(\pi)}$ contained in $U_{\pi}(S)$ always contains $S$ if $M(\pi)$ is dually $\forall$-indefinite. The proof of this will require some preparations. Let $H$ be a subspace such that $H \cap \bar{K}_{0}=(0)$. For $p \in \bar{D}+i H$ we set

$$
\left.d_{H}(p)=\sup \left\{\|h\| ; \mathfrak{l}(p)+h \geq 0 \text { (or } p+i h \in \bar{D}_{0}\right)\right\} \text {. }
$$

Then certainly $0 \leq d_{H}(p)<+\infty$ according to Lemma 5.3.1, and further $d_{H}(p)=0$ if $p \in D_{0}$.

Lemma 5.3.2. The function $d_{H}$ is continuous at each point of $D_{0}$.

Proof. Suppose that $d_{H}$ is not continuous at $p_{0} \in D_{0}$, that is, there 
exists $\delta>0$ and a sequence $\left\{p_{\nu}\right\}_{\nu=1}^{\infty} \subset \bar{D}+i H$ converging to $p_{0}$ such that $d_{H}\left(p_{\nu}\right) \geq \delta$. For $\nu=1,2, \cdots$ choose $h_{\nu} \in H$ such that $\mathfrak{I}\left(p_{\nu}\right)+h_{\nu} \in \bar{K}_{0}$, $\left\|h_{\nu}\right\|>\delta / 2$. Then according to Lemma 5.3.1 $\left\{h_{\nu}\right\}_{\nu=1}^{\infty}$ is bounded. Hence we may assume that $h_{\nu}$ converges to some $h_{0} \in H$. The closedness of $\bar{K}_{0}$ implies that $\mathfrak{l}\left(p_{0}\right)+h_{0} \in \bar{K}_{0}$, from which it follows $h_{0}=0$. But this contradicts $\left\|h_{\nu}\right\| \geq \delta / 2>0$.

Q.E.D.

Lemma 5. 3. 3. Suppose that $M(\pi)$ is dually $\forall$-indefinite. If $U$ is an open subset of $\bar{D}$, then the maximal open subset of $\overline{D(\pi)}$ contained in $\tilde{\pi}(U)$ always contains $\pi\left(U \cap D_{0}\right)$.

Proof. Instead of proving the conclusion we shall prove the following equivalent statement: If $p \in D_{0} \cap U$, then $p$ lies in some open subset of $\bar{D}+H^{c}$ contained in $U+H^{c}$.

Suppose $p \in D_{0} \cap U$. Set $H=\operatorname{Ker} \pi$. For $q \in \bar{D}+i H$ the set

$$
S(q)=q+i\left\{h \in H:\|h\| \leq d_{\bar{B}}(q)\right\}
$$

certainly intersects $\bar{D}$ by the definition of $d_{H}$. Now choose an open subset $\widetilde{U}$ of $N^{0}$ such that $U=\bar{D} \cap \widetilde{U}$. Since $p \in D_{0}, S(p)=\{p\} \subset \widetilde{U}$. Therefore, according to the continuity of $d_{B}$ at $p$, there exists a neighbourhood $\widetilde{V} \ni p$ in $N^{0}$ such that $S(q) \subset \widetilde{U}$ when $q \in(\bar{D}+i H) \cap \widetilde{V}$. But then $S(q) \cap U=S(q) \cap \widetilde{U} \cap \bar{D}=S(q) \cap \bar{D} \neq \phi$. Thus $U \cap(q+i H) \neq \phi$, that is, $q \in U+i H$. Thus $\widetilde{V} \cap(\bar{D}+i H) \subset U+i H$ and this implies the required statement.

In view of this lemma $S$ lies in the maximal open subset of $\overline{D(\pi)}$ contained in $U_{\pi}(S)$, which we shall denote by $V_{\pi}(S)$. Then it follows from Theorem 5.3.1 that the restriction map

$$
\widetilde{H}\left(V_{\pi}(S)\right) \rightarrow \Gamma_{S}\left(\mathscr{H}_{\mathcal{S}}\right)
$$

is a topological linear isomorphism. This and Theorem 5.2.1 imply the following stronger form of the solution of Problem $H$ mentioned in the introduction for stable standard real submanifolds.

Theorem A. If $M(\pi)$ is a stable standard real submanifold, then, for any open subset $S$ of $M(\pi)$, there exists an open subset $U$ 
of the closure of the domain $D(\pi)$ such that $S \subset U$, and the restriction map

$$
\widetilde{H}(U) \rightarrow \Gamma_{S}\left(\mathscr{H}_{\mathcal{S}}\right)
$$

is a topological linear isomorphism (onto $\Gamma_{\mathcal{S}}\left(\mathcal{H}_{\mathcal{S}}\right)$ ).

Now we shall devote tile rest for the study of nondegenerate Siegel domains of the secord kind. Each of them is $D(\pi)$ for some map $\pi: H(V) \rightarrow W$, taking suitable $V$ and $W . M(\pi)$ is the Silov boundary of $D(\pi)$ then. First of all we begin with the definition. Let $W$ be a real vector space and $K$ an open convex cone in $W$ which does not contain any line. Further let $V$ be a complex vector space. A $W^{c}$. valued sesqui-linear fcrm $F(x, y)(x, y \in V)$ is called $K$-hermitian form on $V$ if

$$
\begin{aligned}
& F(x, y)=F(y, x)^{*} \\
& F(x, x) \in \bar{K} \\
& F(x, x)=0 \Rightarrow x=0
\end{aligned}
$$

where the map $\alpha \rightarrow \alpha^{*}$ is the conjugation of $W^{c}$ fixing $W$. Denote by $D(F, K)$ the domain in $V \times W^{c}$

$$
\left\{(x, \alpha) ; \frac{1}{2 i}\left(\alpha-\alpha^{*}\right)-F(x, x) \in K\right\} .
$$

If $D(F, K)$ is affine homogeneous, that is, the group of affine transformation leaving $D(F, K)$ invariant operates transitively on $D(F, K)$, $D(F, K)$ is called a Siegel domain of second kind. If moreover the set $\left\{\frac{1}{i}(F(x, y)-F(y, x))\right\}$ generates $W$, we call $D(F, K)$ non-degenerate. For a non-degenerate Siegel domain we shall assign a fundamental Lie algebra $\mathfrak{m}(F)$ setting

$$
\begin{aligned}
& g_{1}=V, \quad g_{1}=W, \quad \mathfrak{m}=g_{1}+g_{2}, \\
& {\left[g_{2}, g_{1}\right]=\left[g_{2}, g_{2}\right]=(0),} \\
& {[x, y]=\frac{1}{i}(F(x, y)-F(y, x)) \quad x, y \in g_{1} .}
\end{aligned}
$$

Then as proved in $\S 1$ we obtain a (unique) linear mapping $\pi$ of $H(V)$ onto $W$ such that $\mathfrak{m}(\pi)=\mathfrak{m}(F) . \quad M(\pi)$ is then the Šilov boundary of 
$D(F, K)$. (See [7].)

Lemma 2. The map $\pi$ being as above, $D(\pi)=D(F, K)$.

Proof. Let $F_{0}$ denote the $K_{0}$-hermitian form on $V$ given by

$$
F_{0}(x, y)=\frac{1}{2}([I x, y]+i[x, y]) \quad x, y \in V
$$

where [, ] denotes the bracket operation of $\mathfrak{m}^{0}=V+H(V)$. Then

$$
D=D\left(F_{0}, K_{0}\right)
$$

and $D$ is certainly non-degenerate. Since $\bar{K}_{0}$ is the convex closures of the set of positive semi-definite hermitian forms of rank 1, condition (2) implies that $\pi\left(\bar{K}_{0}\right) \subseteq \bar{K}$. Hence $\pi\left(K_{0}\right) \subseteq K$. Thus $D(\pi) \subseteq D(F, K)$.

In order to prove $D(\pi) \supseteq D(F, K)$ we need the following theorem of Piatetski-Šapiro [8].

Theorem P. Every affine transformation of $D(F, K)$ is of the following form

$$
\begin{aligned}
& \alpha \rightarrow A \alpha+2 i F(B x, b)+i F(b, b) \\
& x \rightarrow B x+b
\end{aligned}
$$

where $A \in \operatorname{End}(W) \quad B \in \operatorname{End}_{c}(V)$ and $A F(x, y)=F(B x, B y)(x, y$ $\in V$ ).

Suppose that $g$ is the affine automorphism of $D(F, K)$ indicated in the above theorem. If we denote by $S^{2}(B)$ the restriction to $H(V)$ of $B \otimes B$, then the map $g_{0}$ of $N^{0}=V \oplus H(V)^{c}$ given by

$$
\begin{aligned}
& \alpha \rightarrow S^{2}(B) \alpha+2 i F_{0}(B x, b)+i F_{0}(b, b) \\
& x \rightarrow B x+b
\end{aligned}
$$

is an affine automorphism of $D=D\left(F_{0}, K_{0}\right)$. Thus we obtain a groupisomorphism $g \rightarrow g_{0}$ of the affine automorphism group of $D(F, K)$ into that of $D$ such that $\tilde{\pi} \circ g_{0}=g \circ \tilde{\pi}$. Therefore any orbit of the affine automorphism group of $D(F, K)$ is contained in the irrage by $\tilde{\pi}$ of an orbit of the affine automorphism group of $D$. In paticu'ar $D(\pi)$ $\supseteq D(F, K)$.

Q.E.D.

Now we shall prove that the Šilov bourdary of a non-gere-ate 
Siegel domain $D(F, K)$ is dually totally indefinite. Since $K$ does not contain any line, there exists a hyperplane $P$ of $W$ such that $P \cap \bar{K}$ $=(0)$. Suppose that $P$ is given by $P=\{\alpha \in W: \mathfrak{l}(\alpha)=0\}$ where $\mathfrak{l} \in W^{*}$. Without loss of generality we may assume that $x \in K \Rightarrow l(x)>0$. Then the hermitian form

$$
\mathfrak{l}(F(x, y)) \quad x, y \in V
$$

is certainly positive definite. In fact if

$$
\mathfrak{l}(F(x, x))=0,
$$

then $F(x, x) \in P \cap \bar{K}=(0)$, i.e., $F(x, x)=0$ and by condition (3) we obtain $x=0$. Thus we conclude that the Šilov boundary of $D(F, K)$ is dually totally indefinite.

\section{References}

[1] Dines, L. L., On linear combinations of quadratic forms, Bull. Amer. Math. Soc. 49 (1943), 383-393.

[2] Greenfield, S. J., Cauchy-Riemann equations in several variables, Ann. Scuola Norm. Sup. Pisa 22 (1968), 175-314.

[3] Hörmander, L., Hypoelliptic second order differential equations, Acta Math. 119 (1967), 147-171.

[4] Hua, L. K., Harmonic analysis of functions of several complex variables in the classical domains, Transl. Math. Monogr. 6, Amer. Math. Soc., 1963.

[5] Lewy, H., On the local character of the solutions of an atypical linear differential equation in three variables and a related theorem for regular functions of two complex variables, Ann. of Math. 64 (1956), 514-522.

[6] - On hulls of holomorphy, Comm. Pure Appl. Math. 13 (1960), 587-591.

[7] Naruki, I., An analytic study of a pseudo-complex-structure, to appear in Proc. Intern. Conf. Func. Anal., Tokyo, 1969.

[8] Piatetski-Šapiro, Géometrie des domaines classiques et théorie des fonctions automorphes, Travaux et Recherches Mathématiques, Dunod, Paris, 1966.

[9] Tanaka, N., Graded Lie algebras and geometric structures, Proc. U.S.-Japan Seminar Diff. Geometry (1965), 147-150.

[10] - On generalized graded Lie algebras and geometric structures I, J. Math. Soc. Japan 19 (1967), 215-254.

[11] — On differential systems, graded Lie algebras and pseudo-groups, to appear.

[12] Wells, R. O., On the holomorphic hull of a real submanifold in several complex variables, Comm. Pure Appl. Math. 19 (1966), 145-165.

[13] - Holomorphic hulls and holomorphic convexity of differentiable submanifolds, Trans. Amer. Math. Soc. 132 (1968), 245-262. 
